\title{
III-V Ferromagnetic Semiconductors
}

\author{
F. Matsukura ${ }^{\mathrm{a}, \mathrm{b})}, \mathrm{H} . \mathrm{Ohno}^{\mathrm{a})}$, and T. Dietl ${ }^{\mathrm{a}, \mathrm{b})}$ \\ a) Laboratory for Electronic Intelligent Systems, Research Institute of Electrical Communication, Tohoku University, Sendai, Japan

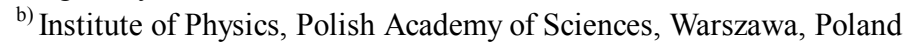

\section{CONTNETS}

\section{Introduction}

\section{Preparation of III-V magnetic semiconductors by} molecular beam epitaxy

$2.1(\mathrm{Ga}, \mathrm{Mn}) \mathrm{As}$

$2.2(\mathrm{In}, \mathrm{Mn}) \mathrm{As}$ grown on $\mathrm{GaAs}$

2.3 (In,Mn)As grown on $(\mathrm{Al}, \mathrm{Ga}) \mathrm{Sb}$

2.4 Other III-V magnetic semiconductors

3. Lattice properties

3.1. Lattice constants

3.2. Local lattice configuration (EXAFS)

3.3. Atomic-scale observations by scanning tunneling microscopy (STM)

\section{Spin and charge states of Mn in III-V magnetic} semiconductors

4.1. Electron spin resonance (ESR)

4.2. Optical spectroscopy

4.3. X-ray magnetic circular dichroism (XMCD)

4.4. Photoemission

\section{Magnetic properties}

5.1. Magnetization

5.2. Magnetic anisotropy

5.3. Magnetic domains

5.4. Cantilever magnetometry

6. Magnetotransport properties

6.1. (Ga,Mn)As

6.1.1. The Hall effect

6.1.2. Temperature and magnetic field dependence of resistivity

6.2. (In,Mn)As
6.2.1. n-type (In,Mn)As
6.2.2. p-type (In,Mn)As
6.2.3. Pseudomorphic p-type (In,Mn)As

6.3. Infrared and far infrared optical conductivity

6.4. Cyclotron resonance

7. Magneto-optical properties

7.1. Faraday rotation

7.2. Magnetic circular dichroism (MCD)

8. Origin of ferromagnetism

8.1. First-principles studies

8.2. Parameterized Hamiltonians

8.3. Hole states and hole mediated exchange interactions

8.4. Mean-field Zener model and its application to
1

5

5

7

(Ga,Mn)As

8.5. Comparison of theoretical and experimental results

8.6. Limitations and refinements of the mean-field Zener model

9. Heterostructures

9.1. Basic properties of heterostructures 29 9.1.1. Structural properties of multilayer structures

9.1.2. Magnetic properties of superlattices, thin films, and quantum well 29

9.1.3. Band offset between (Ga,Mn)As and GaAs 30

9.2. Spin-dependent scattering, interlayer coupling, and tunnel magnetoresistance in trilayer structures 31

9.3. Resonant tunneling diodes (RTDs) 32

9.4. Spin-injection in ferromagnetic semiconductor heterostructures

9.5. Photo-induced ferromagnetism in ( $\mathrm{In}, \mathrm{Mn}) \mathrm{As} / \mathrm{GaSb}$

9.6. Electric-field control of ferromagnetism in gated structures

9.7. Ferromagnetic imprinting

10. Ferromagnetic semiconductors at room temperature 35

10.1. Theoretical suggestions 36

10.2. Cautionary remarks 36

10.3. Experimental results $\quad 37$

10.3.1. (Ga,Mn)As 37

10.3.2. (Ga,Mn)N 37

10.3.3. (Cd,Mn) $\mathrm{GeP}_{2}$

10.3.4. Co-doped $\mathrm{TiO}_{2}$

10.3.5. Co-doped $\mathrm{ZnO}$

10.3.6. Zinc-blende CrAs and $\mathrm{CrSb}$

11. Summary and outlook 38

$\begin{array}{ll}\text { Acknowledgements } & 38\end{array}$

$\begin{array}{ll}\text { References } & 38\end{array}$

\section{Introduction}

Modern information technology utilizes the charge degree of freedom of electrons in semiconductors to process the information and the spin degree of freedom in magnetic materials to store the information. Magnetoelectronics is a new fastly developing field, where the two degrees of freedom, the charge and the spin of the carriers, are utilized simultaneously to 
create new functionalities. In more general terms, this new field is referred to as spin-electronics or spintronics to include those spin-utilizing devices that need neither the magnetic field nor magnetic materials. The magnetoresistance (MR) sensors made of multilayers containing metal ferromagnets, showing giant magnetoresistance (GMR) or tunneling magnetoresistance (TMR), are today's best known successful magnetoeloectronics devices based on the interplay between the two degrees of freedom (Prinz 1998, De Boeck and Borghs 1999, Wolf 2000, Ball 2000, Ziese and Thornton 2001, Wolf et al. 2001).

In semiconductor devices, the spin of carriers has played a minor role so far because the most-well established semiconductor devices based on $\mathrm{Si}$ and $\mathrm{GaAs}$ are non-magnetic and show only negligible effects of spin. On the other hand, from the physical points of view, the enhanced spin-related phenomena due to the coexistence of the magnetism and semiconductor properties have been recognized in magnetic semiconductors and diluted magnetic semiconductors (DMS) (or semimagnetic semiconductors; SMSC) since the 60s. The family of magnetic semiconductors encompasses europium and chromium chalcogenides (rock-salt type: EuSe, EuO and spinels: $\mathrm{CdCr}_{2} \mathrm{~S}_{4}, \mathrm{CdCr}_{2} \mathrm{Se}_{4}$ ), which show ferromagnetic order at low temperatures with the Curie temperature $T_{\mathrm{C}} \leq 100 \mathrm{~K}$. They have been extensively studied, because of their peculiar properties resulting from the exchange interaction between itinerant electrons and localized magnetic spins (s-f and s-d exchange interactions) (Kasuya and Yanase 1968, Methfessel and Mattis 1968, Mauger and Gotard 1986). Owing to these interactions, magnetic semiconductors exhibit a rich variety of striking optical and transport phenomena, which are strongly affected by the magnetic field and the magnetic order, particularly near the metal-to-insulator transition (MIT). However, difficulties in material preparation and in fabrication of heterostructures make this family of compounds less attractive from the application point of view. Manganites (perovskite: (La, $\mathrm{Sr}) \mathrm{MnO}_{3}$ and related materials), which show colossal magnetoresistance (CMR), are magnetic semiconductors, whose studies have been particularly active over the recent years. Their ferromagnetic order, beginning at $\sim 350 \mathrm{~K}$, originates from the double-exchange interaction. Properties of manganites and their epitaxial heterostructures are currently studied aggressively (Coey et al. 1999, Tokura and Tomioka 1999, Tokura 2000). Their compatibility to the well-established electronic devices is an open issue because of the differences in both crystal structure and constituting elements.

DMS are based on non-magnetic semiconductors, and are obtained by alloying them with a sizable amount (a few percents or more) of magnetic elements, such as Mn. The studies of DMS and their heterostructures have offered a wide variety of materials and structures, making it possible to explore further the effect of the exchange interaction in semiconductors. Most of the work had been centered around II-VI based materials such as $(\mathrm{Cd}, \mathrm{Mn}) \mathrm{Te},(\mathrm{Zn}, \mathrm{Co}) \mathrm{S},(\mathrm{Hg}, \mathrm{Fe}) \mathrm{Se}$, where the valence of group II cations is identical to that of most magnetic transition metals (Furdyna and Kossut 1988, Kossut and Dobrowolski 1993, Dietl 1994). Although this made them relatively easy to prepare, difficulties in doping of II-VI-based DMS to either por n-type as well as relatively weak bonds made these materials less attractive for applications. The magnetic properties of II-VI
DMS are dominated by the antiferromagnetic super-exchange interactions among the localized spins, which result in paramagnetic, spinglass or antiferromagnetic behavior depending on the concentration of the magnetic ions and temperature. Recent progress in doping of II-VI materials is gradually changing this situation (Shibata et al. 1988, Baron et al. 1994), for example, hole mediated ferromagnetism was found in p-type II-VI DMS with $T_{\mathrm{C}}<10 \mathrm{~K}$ (Haury et al. 1997, Ferrand et al. 2001, Hansen et al. 2001). Understanding of the carrier-mediated ferromagnetism in semiconductors was put forward by a study of ferromagnetism in IV-VI DMS such as ( $\mathrm{Pb}, \mathrm{Sn}, \mathrm{Mn}) \mathrm{Te}$ (Story et al. 1986). However, IV-VI DMS and their heterostructures are again rather difficult to prepare.

An approach compatible with the present-day electronic materials is to make non-magnetic semiconductors magnetic, and even ferromagnetic, by introducing a high concentration of magnetic ions. III-V compound semiconductors are widely used for high-speed electronic devices as well as for optoelectronic devices. Moreover, heterostructures based on the $\mathrm{GaAs} /(\mathrm{Al}, \mathrm{Ga}) \mathrm{As}$ systems have proven to be a convenient test bench for a new physics and device concepts. Introduction of magnetic III-V compounds opens, therefore, up the possibility of using a variety of magnetic and/or spin-dependent phenomena, not present in the conventional non-magnetic III-Vs, in the optical and electrical devices already established (Fig. 1). The proposal of III-V based magnetic semiconductors with various sets of host materials and transition metals was put forward in 1970s (Gałąka 1977), and some experimental studies were then initiated. At that time, however, III-V materials with a sizable concentration of uniformly distributed magnetic elements were not obtained due to the low solubility of transition metals in III-V semiconductors (Aliyev et al. 1980). The application of non-equilibrium methods to grow III-V-based DMS was rewarded by successful molecular beam epitaxy (MBE) of uniform (In,Mn)As films on GaAs substrates (Munekata et al. 1989). Subsequent discovery of the hole-induced ferromagnetic order in p-type (In,Mn)As (Ohno et al. 1992) encouraged researchers to investigate GaAs-based

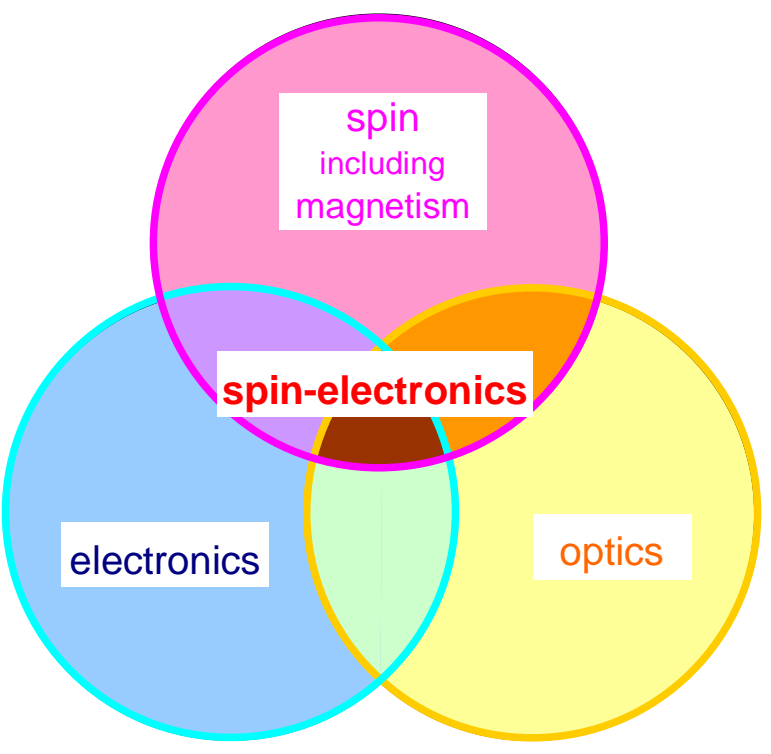

Fig. 1. Concept of spin-electronics (spintronics). In semiconductor spin-electronics spin properties as well as electronic and optical properties are utilized at the same time. 
system (De Boeck et al. 1996) and led to the successful growth of ferromagnetic (Ga,Mn)As (Ohno et al. 1996a). Currently, a number of groups is working on the MBE growth of (Ga,Mn)As and related heterostructures to advance the understanding of this new class of materials (Ohno et al. 1996a, Ohno 1999, Hayashi et al. 1997a, Nishikawa et al. 1997, Van Esch et al. 1997, Sadowski et al. 1998, Kawakami et al. 2000, Potashnik et al. 2001, Schott et al. 2001, Liu et al. 2001).

This chapter reviews the properties of ferromagnetic III-Vs, and is organized in the following way. Section 2 describes the preparation of ferromagnetic III-Vs, and is followed by the presentation of lattice properties in Section 3. In Section 4, spin and charge states of magnetic ions in III-V DMS are discussed. The magnetic and magnetotransport properties are presented in Section 5 and 6, respectively. Section 7 summarizes optical and magneto-optical properties. Possible origin of ferromagnetism in III-V-based DMS is discussed in Section 8. Properties of heterostructures are presented in Section 9 and recent developments of room-temperature ferromagnetic semiconductors are introduced in Section 10. Summary and outlook is given in Section 11.

\section{Preparation of III-V magnetic semiconductors by molecular beam epitaxy}

In order to observe magnetic cooperative phenomena in diluted magnetic systems, one needs to introduce a sizable amount of magnetic elements (a few percents or more), which is beyond their solubility limit in III-V semiconductors. Although non-equilibrium epitaxial growth methods such as molecular beam epitaxy (MBE) could offer doping in excess of the thermodynamic solubility limit, segregation of impurities during MBE growth was an obstacle in obtaining high concentrations of magnetic elements (DeSimone 1982). It was demonstrated that low temperature MBE (LT-MBE, growth temperature $T_{\mathrm{S}}<$ $300^{\circ} \mathrm{C}$ ) can lead to successful epitaxy of (In,Mn)As with a few percents of $\mathrm{Mn}$, in which the solubility limit is overcome as well as the segregation phenomena and the formation of unwanted second phases is suppressed (Munekata et al. 1989). InAs was chosen as a host compound because it was thought to be a suitable material for low temperature growth due to its relatively small bond energy.

\section{1. (Ga,Mn)As}

Typical MBE growth of (Ga,Mn)As is carried out by using solid source MBE with elemental sources Ga, Mn, In, Al and As, usually without intentional doping. Mn provides both localized spins and holes due to its acceptor nature. Epitaxial films of (Ga,Mn)As are grown on semi-insulating GaAs (001) substrates at a typical growth rate of 0.6-0.8 $\mu \mathrm{m} /$ hour under As-stabilized conditions. Normally, either a GaAs buffer layer or an (Al,Ga)As buffer layer is grown before epitaxy of (Ga,Mn)As. To control strain in the film, strain-relaxed thick (In,Ga)As $(\sim 1$ $\mu \mathrm{m})$ with the lattice constant $a_{\mathrm{o}}$ greater than the subsequent (Ga,Mn)As layer can be employed. The Mn composition $x$ in the $\mathrm{Ga}_{1-x} \mathrm{Mn}_{x}$ As films can be determined from measurements of $a_{\mathrm{o}}$ by x-ray diffraction (XRD), once the dependence $a_{0}(x)$ is calibrated by other means, such as electron probe micro-analysis
(EPMA) or secondary ion mass spectroscopy (SIMS).

The growth of (Ga,Mn)As can be initiated by simply commencing the Mn flow during the LT-GaAs growth and keeping the substrate temperature $T_{\mathrm{S}}$ constant at $250^{\circ} \mathrm{C}$. No special precautions are needed at the start of $(\mathrm{Ga}, \mathrm{Mn})$ As growth. However, properties of $(\mathrm{Ga}, \mathrm{Mn}) \mathrm{As}$ films do depend on the growth parameters such as As overpressure and $T_{\mathrm{S}}$ (Matsukura et al. 1998a, Shimizu et al. 1999, Schott et al. 2001). This may stem from the influence of these parameters on the degree of compensation of the Mn acceptors by deep donors, primarily As antisites which are known to be present with a high concentration in LT-GaAs (Look 1991, Luysberg et al. 1998). However, as long as the established growth procedure is followed, the properties of (Ga,Mn)As films are reproducible; for example, for a given Mn composition $x$, the ferromagnetic transition temperature $T_{\mathrm{C}}$ can always be maintained in the range of $2000 x \pm 10 \mathrm{~K}$ (Matsukura et al. 1998b).

Reflection high-energy electron diffraction (RHEED) patterns are used to monitor the surface reconstruction during the growth. RHEED of GaAs [or (Al,Ga)As] buffer layer grown at $T_{\mathrm{S}} \sim 570^{\circ} \mathrm{C}$ shows the well-know $(2 \times 4)$ pattern, which changes to the $\mathrm{c}(4 \times 4)$ pattern when $T_{\mathrm{S}}$ is lowered to $480-520^{\circ} \mathrm{C}$, and remains $\mathrm{c}(4 \times 4)$ below. Whereas the RHEED pattern of GaAs layers grown at $T_{\mathrm{S}}=250^{\circ} \mathrm{C}$ shows a $(1 \times 1)$ pattern (no reconstruction) (Fig. 2a), that of $(\mathrm{Ga}, \mathrm{Mn}) \mathrm{As}$ is $(1 \times 2)$ during and after the growth (Fig. 2b). The origin of this difference is not yet clear. When the Mn flux and/or $T_{\mathrm{S}}$ are too high, the RHEED pattern indicates the appearance of a second phase on the surface, which is MnAs in the hexagonal NiAs-structure (Fig. 2c). When $T_{\mathrm{S}}$ is too low, spotty RHEED pattern appears showing that the growth mode changes from the two-dimensional (2D) to three-dimensional (3D), resulting in a polycrystalline material (Fig. 2d). The maximum value of $x$ obtained so far is about 0.07 at $250^{\circ} \mathrm{C}$, and increases up to $\sim 0.10$ at $T_{\mathrm{S}}=200^{\circ} \mathrm{C}$ (Takamura et al. 2001). Attempts to increase $x$ even further have so far been unsuccessful because of the surface segregation that occurs even at low growth temperatures. At a fixed value of $x=0.035$, epitaxial (Ga,Mn)As films can be grown at $T_{\mathrm{S}}$ varying from 160 to $320^{\circ} \mathrm{C}$ (Shen et al. 1997a).

Clear RHEED oscillations are observed at the initial growth stage (also without $\mathrm{Mn}$ ) under the conditions appropriate for (Ga,Mn)As epitaxy. Thus, the growth is two-dimensional,
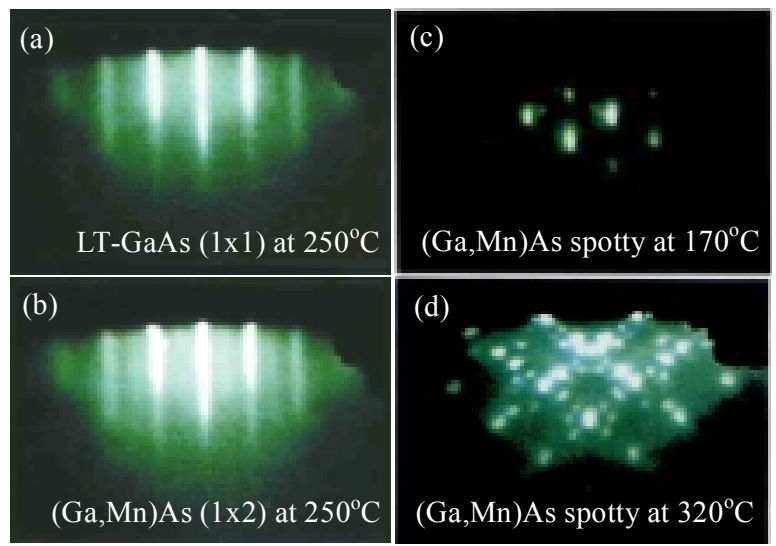

Fig. 2. Reflection high energy electron diffraction (RHEED) patterns taken from $\left[\begin{array}{ll}\overline{1} & 10\end{array}\right]$ azimuth. (a) Low-temperature grown $\mathrm{GaAs}$ at $250^{\circ} \mathrm{C}$, (b) $(\mathrm{Ga}, \mathrm{Mn}) \mathrm{As}$ at $250^{\circ} \mathrm{C}$, (c) $170^{\circ} \mathrm{C}$, and (d) $320^{\circ} \mathrm{C}$ (Shen et al. 1997a). 


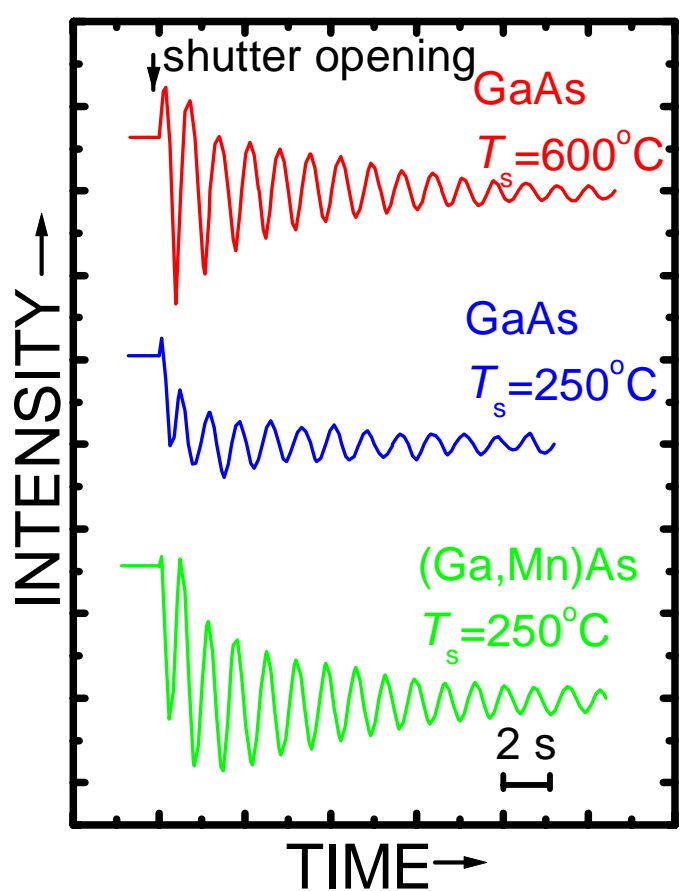

Fig. 3. Temporal evolution of RHEED specular beam intensity (along [110] azimuth) for $\mathrm{GaAs}$ at $600^{\circ} \mathrm{C}, \mathrm{GaAs}$ at $250^{\circ} \mathrm{C}$, and $(\mathrm{Ga}, \mathrm{Mn}) \mathrm{As}$ at $250^{\circ} \mathrm{C}$ from top to bottom (Shen et al. 1997a).

and its rate can be determined from the oscillation period (Fig. 3) (Shen et al. 1997a, b). Monte Carlo simulations of RHEED oscillations have been carried out for LT-GaAs employing the cubic solid-on-solid model (Yasuda and Ohno 1999). The results show that the RHEED oscillations are related to an enhancement in migration of Ga adatoms caused by a surfactant effect of the excess As overlayer. The surfactant effect of As is demonstrated also by the surface visualization (Guo et al. 2000, Tazima et al. 2001a). Scanning tunneling microscopy (STM) study of LT-GaAs shows that the step density on the surface increases monotonically with the decrease of $T_{\mathrm{S}}$, indicating the monotonous decrease of $\mathrm{Ga}$ migration length. This result suggests that the origin of RHEED oscillation may also relate to the decrease of the Schwoebel barrier height due to the formation of small growth islands (Tazima et al. 2001b). The enhancement of the RHEED oscillation of (Ga,Mn)As is observed, when the lattice-relaxed (In,Ga)As is used as a buffer layer (Matsukura 1997) or (Ga,Mn)As contains a few percents of In (Katsumoto 1999). In these cases, it is considered that In atoms act as additional surfactant.

A schematic phase diagram of MBE growth is depicted in Fig. 4 (Ohno 1998, Shen et al. 1999). Recently it was shown that metallic (Ga,Mn)As with $x=0.1$ can be obtained by the use of a modified $\mathrm{MBE}$ growth technique at $T_{\mathrm{S}}=150^{\circ} \mathrm{C}$, migration-enhanced epitaxy (MEE), where the beam fluxes of source materials are precisely controlled (Sadowski et al. 2001a, 2001b).

The surface morphology investigated by atomic-force microscopy (AFM) shows that (Ga,Mn)As with 2D growth mode has a flat surface being comparable with a GaAs surface [root-mean-square (RMS) of the roughness $<0.5 \mathrm{~nm}$ ]. On the o ther hand, (Ga,Mn)As with segregated RHEED pattern has a rather rough surface, characterized by about 10 times larger RMS, which is probably due to small MnAs clusters (Yang et al. 2000).

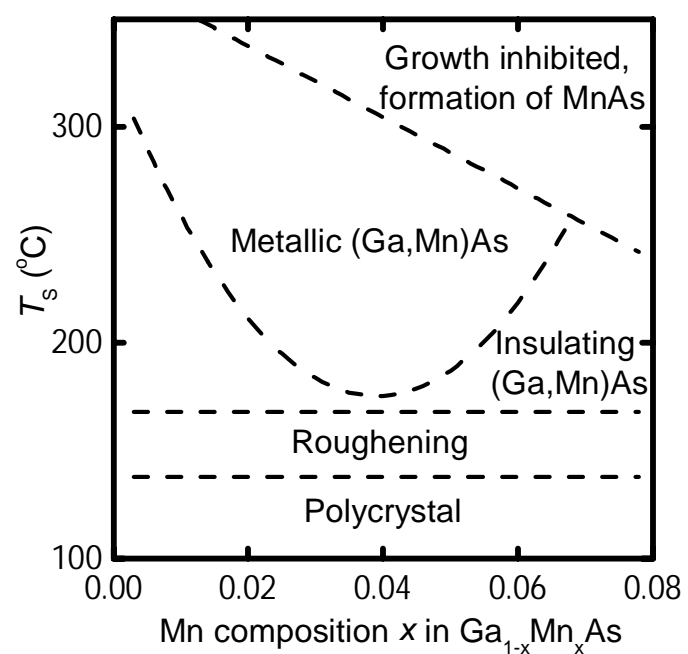

Fig. 4. Schematic diagram of properties of (Ga,Mn)As films in relation to the growth parameters. Lines provide a rough guide (Ohno 1998, Shen et al. 1999).

The homogeneity of as-grown (Ga,Mn)As and the precipitation of MnAs clusters after annealing at around $600^{\circ} \mathrm{C}$ are confirmed by transmission electron microscopy (TEM) (De Boeck et al. 1996). The disappearance of the (Ga,Mn)As phase by annealing at temperatures above $400^{\circ} \mathrm{C}$ is also confirmed by the disappearance of x-ray diffraction peaks of $(\mathrm{Ga}, \mathrm{Mn}) \mathrm{As}$ (Shen et al. 1997a). Low-temperature annealing at $\sim 300^{\circ} \mathrm{C}$ changes lattice constant, magnetic, and electrical properties of (Ga,Mn)As, which is thought to result from the evaporation of excess As atoms that form complexes with Mn acceptors (Hayashi et al. 2001, Potashnik et al. 2001). By additional doping of high concentration of Sn, n-type (Ga,Mn)As can be also grown (Satoh et al. 1997, 2001).

It has been shown that digital alloys, multilayer structures consisting of GaAs and less-than 1 monolayer (ML)-thick MnAs, which exhibit the ferromagnetic order at low temperatures, can be grown by atomic-layer epitaxy (ALE) (Chen et al. 2000, Kawakami et al. 2000). The growth of (Ga,Mn)As with nominal $\mathrm{Mn}$ composition up to 0.04 by metal-organic vapor-phase epitaxy (MOVPE) has also been carried out. MOVPE-grown (Ga,Mn)As includes MnAs microclusters due to the high-growth temperature of $400-600^{\circ} \mathrm{C}$, which is necessary for the decomposing of precursor compounds (Hartmann et al. 2000, Heimbrodt et al. 2001). MBE growth of (Ga,Mn)As on GaAs (411)A and Si (001) substrates and (In,Ga,Mn)As on InP (001) substrates and the observation of their ferromagnetism also have been carried out (Omiya et al. 2002, Zhao et al. 2001a, Ohya et al. 2001, Slupinski et al. 2002a).

\section{2. (In,Mn)As grown on GaAs}

The overall trend in the properties of (In,Mn)As grown by MBE can be summarized in terms of $x$ and $T_{\mathrm{S}}$ (Munekata et al. 1989, 1990, 1991, Ohno et al. 1991). When (In,Mn)As layers are grown directly on the GaAs substrate, either n- or p-type conduction is observed depending on $x$ and $T_{\mathrm{S}}$. At $T_{\mathrm{S}}$ of $200^{\circ} \mathrm{C}$, thick (In,Mn)As layers ( $>1 \mu \mathrm{m})$ grown on GaAs (001) substrates are n-type. The anomalous Hall effect, which is proportional to the perpendicular component of the magnetization of the film, has not been observed in n-type films. 


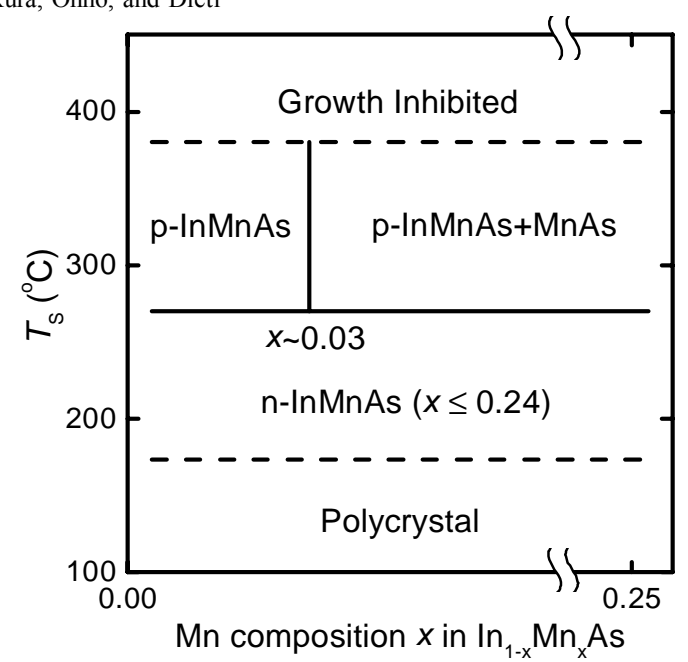

Fig. 5. Schematic diagram of properties of (In,Mn)As films grown directly on GaAs (001) substrate in relation to the growth parameters (Ohno 1991).

Donor-type defect formation in the InAs host lattice at growth temperature is most probably responsible for the n-type conduction in such (In,Mn)As layers. Defect formation may also be enhanced by the high density of mismatch dislocations in the (In,Mn)As layers; the lattice mismatch in question is about $7 \%$. When $T_{\mathrm{S}}<200^{\circ} \mathrm{C}$, polycrystalline growth occurs.

At higher $T_{\mathrm{S}}$ of $400^{\circ} \mathrm{C}$, the layers are p-type at low $x(<$ 0.03 ). MnAs ferromagnetic clusters start to form above $x=0.03$, and the films become eventually n-type at high $x$ values of 0.18 . The hole concentration is identical to the Mn concentration up to $x=0.004\left(p=7 \times 10^{19} \mathrm{~cm}^{-3}\right)$, at which the hole concentration peaks and then rolls off with the increase of $x$. Thus, in the low $x$ region of thick p-type layers grown at $300^{\circ} \mathrm{C}$, Mn behaves as a shallow acceptor. At $x=0.026$ the hole concentration is $3 \times 10^{18}$ $\mathrm{cm}^{-3}$. No epitaxy is observed at $T_{\mathrm{S}}>400^{\circ} \mathrm{C}$. The phase diagram showing the relation between film properties and growth parameters is shown in Fig. 5 (Ohno et al. 1991). Low-temperature annealing of (In, Mn)As results in an increase of $p$ and $T_{\mathrm{C}}$, similar to (Ga,Mn)As (Endo et al. 2001, Hashimoto et al. 2002).

The homogeneity of (In,Mn)As films grown at $\sim 200^{\circ} \mathrm{C}$ and the existence of MnAs clusters in the samples grown at $\sim 300^{\circ} \mathrm{C}$ is confirmed by TEM observation. The annealing at $450^{\circ} \mathrm{C}$ modifies the size of the clusters (Guha and Munekata 1993, Van Esch et al. 1995).

InAs quantum dots (QDs) fabricated by the self-organized growth mode (Stranki-Krastanov mode) have attracted a great deal of interest. The interplay between dimensional confinement and magnetism is certainly an interesting topic to pursue. The formation of self-organized (In,Mn)As dots was demonstrated by growing (In,Mn)As on (001), (211)B, (311)B GaAs substrates by $\mathrm{MBE}$ at $T_{\mathrm{S}}=350^{\circ} \mathrm{C}$ (Guo et al.1998, 1999). In order to obtain uniform structures, the growth temperature should be kept as high as possible, close to the point of the phase separation. Photoluminescence from (In,Mn)As QDs has been observed (Sadowski et al. 2001c).

\section{3. (In, Mn)As grown on $(\mathrm{Al}, \mathrm{Ga}) \mathrm{Sb}$}

Contrary to the results of thick layers directly grown on GaAs substrate, thin (In,Mn)As layers $(<30 \mathrm{~nm})$ grown at

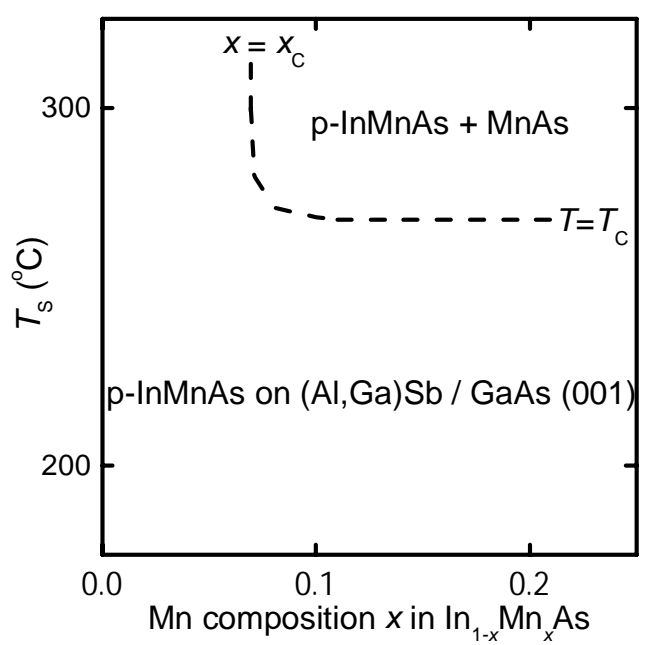

Fig. 6. Schematic diagram of properties of (In,Mn)As films grown on GaAs substrate with thick $(\mathrm{Al}, \mathrm{Ga}) \mathrm{Sb}$ buffer layer between the two in relation to the growth parameters (Munekata 1995).

$200^{\circ} \mathrm{C}$ pseudomorphically on thick (Al,Ga)Sb buffer layers (200 500 nm grown on (001) GaAs substrates) are p-type (Ohno et al. 1992, Munekata et al., 1992). A schematic phase diagram of thin (In,Mn)As layers on $(\mathrm{Al}, \mathrm{Ga}) \mathrm{Sb}$ buffer layers is shown in Fig. 6 (Munekata 1995). The p-type conduction results, most probably, from the acceptor nature of $\mathrm{Mn}$ and low dislocation density in the pseudomorphic thin layers. Although the lattice mismatch between the (In,Mn)As layers and the (Al,Ga)Sb buffer layers is small, depending on the growth procedure different initial growth modes have been observed (Shen et al. 1997c). Careful minimization of As flux is required to obtain 2D growth at the initial stage of (In,Mn)As epitaxy ( $x$ $=0.02 \sim 0.04)$ on $(\mathrm{Al}, \mathrm{Ga}) \mathrm{Sb}$, otherwise $3 \mathrm{D}$ growth takes place, as witnessed by RHEED patterns. It is interesting to note that despite streaky RHEED patterns that appear after 10 20 nm growth of $(\mathrm{In}, \mathrm{Mn}) \mathrm{As}$, the magnetic properties, probed by the anomalous Hall effect, show a correlation with the initial growth mode. In particular, the $3 \mathrm{D}$ nucleation results in superparamagnetism, whereas the 2D nucleation leads to ferromagnetism with square hystreresis (Shen et al. 1997c). The tensile strain associated with the lattice mismatch between ( $\mathrm{In}, \mathrm{Mn}) \mathrm{As}$ and $(\mathrm{Al}, \mathrm{Ga}) \mathrm{Sb}(0.6 \sim 1.3 \%)$ makes the easy axis to be perpendicular to the film plane (Munekata et al. 1993). The increase of the thickness beyond the critical value (about $55 \mathrm{~nm}$ for (In,Mn)As/AlSb) causes lattice relaxation, which affects magnetic anisotropy. The thickness of the (In,Al)As spacer layer (Al composition $\sim 0.15$ ) between ( $\mathrm{In}, \mathrm{Mn}$ )As layer and $\mathrm{AlSb}$ buffer also influences the magnetic properties of (In,Mn)As (Munekata et al. 1992).

\subsection{Other III-V magnetic semiconductors}

With the advances of the non-equilibrium crystal growth techniques, other III-V magnetic semiconductors than (Ga,Mn)As and (In,Mn)As with different host semiconductors and different transition metals have appeared and the investigation of properties of these new materials are underway.

- $(\mathrm{Ga}, \mathrm{Fe})$ As: The growth of $(\mathrm{Ga}, \mathrm{Fe})$ As with $\mathrm{Fe}$ compositions up to $\sim 0.1$ was carried out by LT-MBE at $T_{\mathrm{S}}=260-350^{\circ} \mathrm{C}$ on GaAs substrate (Haneda et al. 2000a). The lattice constant of 
$(\mathrm{Ga}, \mathrm{Fe})$ As becomes smaller with the increase of $\mathrm{Fe}$ composition, reflecting the shorter bond length of Fe-As than that of Ga-As. The elevation of $T_{\mathrm{S}}$ leads to the precipitation of Fe-related clusters. Extended x-ray absorption fine structure (EXAFS) analysis revealed that $\mathrm{Fe}$ atoms in $(\mathrm{Ga}, \mathrm{Fe})$ As grown at low $T_{\mathrm{S}}$ substitute into Ga sites in the zinc-blende structure, whereas that $\mathrm{Fe}$ atoms in high- $T_{\mathrm{S}}$ grown samples form Fe clusters and/or Fe-As complexes (Soo et al. 2001a). Van-Vleck type paramagnetism occurs in $(\mathrm{Ga}, \mathrm{Fe}) \mathrm{As}$, as the temperature dependence of magnetization is rather weak at low temperatures $(<10 \mathrm{~K})$. The conduction type is n-type and a photo-induced MR effect is observed below $100 \mathrm{~K}$. In GaAs-Fe composite structures (GaAs including $\mathrm{Fe}$ clusters), photo-induced magnetization as well as MR effect is observed even at room temperature (Haneda et al. 2000b, 2001). The properties of ( $\mathrm{Ga}, \mathrm{Fe}, \mathrm{Mn})$ As with transition metal compositions up to 0.04 have been investigated. The results show that $(\mathrm{Ga}, \mathrm{Fe}, \mathrm{Mn}) \mathrm{As}$ becomes insulating with the increase of $\mathrm{Fe}$ content, and that there are contributions of ferromagnetic and paramagnetic terms to the film magnetization. The ratio of paramagnetic part to ferromagnetic part increases with the increase of $\mathrm{Fe}$ content (Moriya et al. 2002).

- (Ga,Cr)As: (Ga,Cr)As layers with $\mathrm{Cr}$ compositions up to 0.1 were grown by LT-MBE at $T_{\mathrm{S}}=250^{\circ} \mathrm{C}$ on GaAs substrates (Saito et al. 2001). The magnetization measurements show that ( $\mathrm{Ga}, \mathrm{Cr}$ )As is superparamagnetic and the Curie-Weiss plot has a positive Curie temperature, indicating that the dominant interactions between the $\mathrm{Cr}$ ions are ferromagnetic. The value of the effective magnetic moment of the $\mathrm{Cr}$ ions is consistent with that of the divalence state of $\mathrm{Cr}$, suggesting that the $\mathrm{Cr}$ atoms act as acceptors. Whereas there is a report of p-type conduction of (Ga,Cr)As (Okazawa et al. 1999, 2001), the determination of the conduction type by Hall measurements is rather difficult due to a small Hall voltage, suggesting that the conduction is dominated by hopping. Ferromagnetism below $\sim 45 \mathrm{~K}$ of ( $\mathrm{Ga}, \mathrm{Cr}$ )As with higher $\mathrm{Cr}$ composition (0.14) is also observed. From the photoemission spectroscopy, $(\mathrm{Ga}, \mathrm{Cr})$ As with $\mathrm{Cr}$ compositions larger than 0.25 is metallic (Yamada et al. 2001). Ferromagnetic properties of $(\mathrm{Ga}, \mathrm{Cr}, \mathrm{Mn}) \mathrm{As}$ grown by $\mathrm{MBE}$ are reported (Akinaga et al. 2000a). However, $T_{\mathrm{C}}$ is lower than that of $(\mathrm{Ga}, \mathrm{Mn})$ As with the same Mn composition, which may be due to the compensation effect.

- (Ga,Mn)N: bulk crystals with $x$ up to 0.02 were grown by a resublimation method (Gebicki et al. 2000), and microcrystals with $x$ up to 0.005 were obtained by an ammonthermal method (Zając et al. 2001a). Both compounds show extra Raman peaks induced by disorder stemming from $\mathrm{Mn}$ incorporation. Magnetization measurements reveal that these compounds are paramagnetic and from its temperature dependence antiferromagnetic nearest-neighbor interaction coupling $(\sim-2 \mathrm{~K})$ is obtained (Zając et al. 2001b).

Structural properties of MBE-grown $(\mathrm{Ga}, \mathrm{Mn}) \mathrm{N}$ with $x$ up to 0.09 on AlN buffer onto $\mathrm{Al}_{2} \mathrm{O}_{3}$ substrate grown at $T_{\mathrm{S}}=$ $650-750^{\circ} \mathrm{C}$ by $\mathrm{MBE}$ using RF-nitrogen plasma source have been investigated (Kuwabara et al. 2001a, 2001b). Both cross-sectional TEM and plane-view scanning electron microscopy (SEM) images show no visible second phase. Extended $\mathrm{x}$-ray absorption fine structure (EXAFS) analysis indicates that the Mn atoms are incorporated in the Ga sites. Magnetization measurement revealed that there exist contributions from both ferromagnetic (even at room temperature) and paramagnetic phases. Kuwabara et al. suppose that the ferromagnetic part may originate from the presence of ferromagnetic or ferrimagnetic second phases of Ga-Mn and/or $\mathrm{Mn}-\mathrm{N}$, since the samples are highly resistive and thus no carrier-induced ferromagnetism is expected. According to the Curie-Weiss analysis of the paramagnetic part, the paramagnetic Curie-Weiss temperature $\theta_{\mathrm{p}}$ is negative in the very dilute $\mathrm{Mn}$ composition regime $\left(\theta_{\mathrm{p}} \sim-8 \mathrm{~K}\right.$ with $S \sim 2.5$ for $[\mathrm{Mn}]=7 \times 10^{19}$ $\left.\mathrm{cm}^{-3}\right)$, and changes into positive when the Mn composition is increased $\left(\theta_{\mathrm{p}} \sim 20 \mathrm{~K}\right.$ with $S \sim 2.5$ for $\left.[\mathrm{Mn}]=8 \times 10^{20} \mathrm{~cm}^{-3}\right)$. The EXAFS and near-edge $\mathrm{x}$-ray absorption fine structure (NRXAFS) analyses on the samples prepared by the same authors indicate that the most part of Mn substitutes into the $\mathrm{Ga}$ sites, the valency of $\mathrm{Mn}$ is $2+$, and that there is a possible formation of Mn clusters (Soo et al. 2001b).

An n-type $(\mathrm{Ga}, \mathrm{Mn}) \mathrm{N}$ film with $x=0.07$ on $\mathrm{Al}_{2} \mathrm{O}_{3}$ substrate was grown by $\mathrm{MBE}$ at $865^{\circ} \mathrm{C}$ using a nitrogen plasma source (Overberg et al. 2001). The magnetization at $10 \mathrm{~K}$ is nonlinear as a function of the magnetic field and small hysteresis are visible, indicating that the film is ferromagnetic. Negative magnetoresistance and nonlinear dependence of the Hall resistance are assigned to spin effects, though the negative magnetoresistance is reminiscent of a weak localization effect.

MBE-grown (Ga,Mn)N films with $x=0.06$ and 0.09 were prepared using $\mathrm{NH}_{3}$ as nitrogen source (Sonoda et al. 2001). The results of magnetization measurements are similar to those of Kuwabara et al. However, Sonoda et al. claim that their (Ga,Mn)N films show the ferromagnetic behavior even at room temperature. Indeed, the estimation of $T_{\mathrm{C}}$ from the temperature dependence of magnetization is $940 \mathrm{~K}$, and no $\mathrm{Mn}-\mathrm{Ga}$ and Mn-N compounds with such high $T_{\mathrm{C}}$ have previously been found. (One should note that rather high $T_{\mathrm{C}}(\sim 750 \mathrm{~K})$ has been observed in Mn-Ga alloys (Bither and Cloud, 1965). Very recently, the room temperature ferromagnetism in $(\mathrm{Ga}, \mathrm{Cr}) \mathrm{N}$ (Hashimoto, M. et al. 2002) and (Ga,Mn)P:C (Theodoropoulou et al. 2002) has also been reported.)

The magnetic properties of $\mathrm{p}-\mathrm{GaN}$ implanted with high doses (3 5\%) of $\mathrm{Mn}$ (annealed at $700-1000^{\circ} \mathrm{C}$ after doses) have been investigated. The result shows that the sample is ferromagnetic with $T_{\mathrm{C}} \sim 250 \mathrm{~K}$ (Theodoropoulou et al. 2001a).

$(\mathrm{Ga}, \mathrm{Mn}) \mathrm{N}$ film prepared by post growth Mn doping using solid state diffusion shows ferromagnetic behavior at room temperature, which is confirmed by the observation of an anomalous Hall effect (Reed et al. 2001).

It seems that a considerable amount of work is needed to clarify the structural and magnetic properties of (Ga,Mn)N.

- $(\mathrm{Ga}, \mathrm{Fe}) \mathrm{N}$ : GaN films doped with $\mathrm{Fe}$, with concentrations up to $\sim 3 \times 10^{19} \mathrm{~cm}^{-3}$ were grown by MBE at several $T_{\mathrm{S}}$ from 380 to $520^{\circ} \mathrm{C}$ directly on sapphire (0001) substrates. Ferromagnetic behavior with $T_{\mathrm{C}} \sim 100 \mathrm{~K}$ is observed only in the sample grown at $\sim 400^{\circ} \mathrm{C}$, in spite of quite a low concentration of $\mathrm{Fe}$ (Akinaga et al. 2000b). GaN:Fe films (Fe up to $6 \times 10^{21} \mathrm{~cm}^{-3}$ ) grown by MBE at $T_{\mathrm{S}}=500 \sim 800^{\circ} \mathrm{C}$ show a superparamagnetic behavior (Kuwabara et al. 2001a, 2001b) together with superparamagnetic contributions of possible Ga-Fe and/or Fe-N 
inclusions. The EXAFS analysis suggests that the decrease of $T_{\mathrm{S}}$ leads to a structural transition from wurtzite to zinc-blende structure, and this transition may be related to the origin of ferromagnetism in GaN film with Fe (Ofuchi et al. 2001a). The emission channeling result on annealed Fe implanted $\mathrm{GaN}$ (Fe concentration $10^{17} \sim 10^{18} \mathrm{~cm}^{-3}$ ) shows that the majority of $\mathrm{Fe}$ (80\%) occupies substitutional Ga sites (Wahl et al. 2001). p-GaN implanted with a high dose of $\mathrm{Fe}$ (3 5\%) shows ferromagnetic behavior, $T_{\mathrm{C}} \sim 250 \mathrm{~K}$ (Theodoropoulou et al. 2001b).

- (Ga,Mn)Sb and ( $\mathrm{Ga}, \mathrm{Cr}) \mathrm{Sb}$ : The growth of bulk GaSb crystals heavily doped by $\mathrm{Mn}$ was reported earlier, but whether an alloy between $\mathrm{GaSb}$ and $\mathrm{Mn}$ is formed remains unclear (Aliyev et al. 1980, Adhikari and Basu 1994). GaSb films with a few percent of $\mathrm{Mn}$ or $\mathrm{Cr}$ were also grown by MBE at $T_{\mathrm{S}}=250-560^{\circ} \mathrm{C}$ (Abe et al. 2000, 2001). The surface morphology of MBE-grown samples observed by atomic force microscopy (AFM) shows that these films contain clusters, which may be transition-metal-antimonide compounds. The size of the clusters becomes smaller with the decrease of $T_{\mathrm{S}}$.

For $\mathrm{GaSb}$ with $\mathrm{Mn}$, magnetization measurements show a ferromagnetic behavior even at room temperature, indicating the existence of ferromagnetic $\mathrm{Mn}-\mathrm{Sb}$ clusters. The larger coercive force for higher $T_{\mathrm{S}}$ may reflect the larger size of these clusters. The increase of the magnetization at low-temperatures suggests the formation of ferromagnetic zinc-blende $(\mathrm{Ga}, \mathrm{Mn}) \mathrm{Sb}$. According to the saturation value of magnetization, only about $10 \%$ of the nominal $\mathrm{Mn}$ concentration contributes to the formation of $(\mathrm{Ga}, \mathrm{Mn}) \mathrm{Sb}$ grown at $T_{\mathrm{S}}=560^{\circ} \mathrm{C}$, but $30 \%$ of $\mathrm{Mn}$ contributes to $(\mathrm{Ga}, \mathrm{Mn}) \mathrm{Sb}$ if $T_{\mathrm{S}}=$ $250^{\circ} \mathrm{C}$. Magnetotransport measurements determine the properties of $(\mathrm{Ga}, \mathrm{Mn}) \mathrm{Sb}$ without the influence of $\mathrm{Mn}-\mathrm{Sb}$ clusters, and the data show a ferromagnetic behavior with $T_{\mathrm{C}} \sim$ $10 \mathrm{~K}$ and $\sim 30 \mathrm{~K}$ for $T_{\mathrm{S}}=560^{\circ} \mathrm{C}$ and $250^{\circ} \mathrm{C}$, respectively (Abe et al. 2000).

In the case of $\mathrm{GaSb}$ with $\mathrm{Cr}$, both antiferromagnetic and ferromagnetic contributions are detected. Due to an antiferromagnetic nature of $\mathrm{Cr}$-Sb compounds, the magnitude of the magnetization decreases with the increase of $\mathrm{Cr}$ content, whereas ferromagnetic hysteresis persist even at room temperature. The result of magnetotransport measurements shows that $(\mathrm{Ga}, \mathrm{Cr}) \mathrm{Sb}$ grown at $T_{\mathrm{S}}=550^{\circ} \mathrm{C}$ is antiferromagnetic at $1.5 \mathrm{~K} .(\mathrm{Ga}, \mathrm{Cr}) \mathrm{Sb}$ grown at $250^{\circ} \mathrm{C}$ is highly resistive at low temperatures $(<100 \mathrm{~K})$, which may be due to the compensation of intrinsic holes in epitaxial GaSb films by the $\mathrm{Cr}$ ions (Abe et al. 2001).

Mn doped InSb: The properties of InSb:Mn single crystals with $[\mathrm{Mn}]<3.5 \times 10^{17} \mathrm{~cm}^{-3}$ grown by the Czochralski method have been investigated extensively (Obukhov and Pepic 1989, Obukhov 1993, 1996, Henriques et al. 1999). Mn in InSb behaves as a shallow acceptor (activation energy $E_{\mathrm{a}}=7 \mathrm{meV}$ ), and a metal-insulator transition (MIT) occurs at low Mn concentrations $\left(2 \times 10^{17} \mathrm{~cm}^{-3}\right)$ due to a relatively large Bohr radius of the bound holes. The interactions between the $\mathrm{Mn}$ spins are predominately antiferromagnetic. A strong spin-dependent coupling between the Mn spins and holes gives rise to large magnetoresistance effects, leading to the field-induced insulator-to-metal transition in InSb:Mn at low temperatures (Obukhov 1996, Henriques et al. 1999). An anomalous Hall effect, whose coefficient is larger for lower Mn concentrations $\left(2 \times 10^{16}<[\mathrm{Mn}]<3 \times 10^{17} \mathrm{~cm}^{-3}\right)$, has been observed (Obukhov and Pepic 1989).

\section{Lattice properties}

\subsection{Lattice constants}

X-ray diffraction (XRD) measurements show that (Ga,Mn)As has the zinc-blende structure without detectable second phase. The results for the (004) reflection using $\mathrm{Cu}-\mathrm{K} \alpha$ radiation show that the lattice constant $a$ of $(\mathrm{Ga}, \mathrm{Mn}) \mathrm{As}$ increases with the increase of $x$ as shown in Fig. 7a (Ohno et al. 1996a). Asymmetric double-crystal XRD on (224) or (115) reflection demonstrates that the (Ga,Mn)As films are fully strained at least up to $2 \mu \mathrm{m}$ (Fig. 7c) (Shen et al. 1999). The reciprocal space mapping on the (004) plane confirms also that the (Ga,Mn)As layers are fully strained (Sadowski et al. 2000). This rather high critical thickness is probably due to the low growth temperature, which prevents dislocations from nucleating (Price 1991). The direction of the strain can be controlled by using a thick lattice-relaxed (In, Ga)As buffer layer, which has a larger lattice constant than that of (Ga,Mn)As as shown in Fig. 7b (Ohno et al. 1996b). The peak corresponding

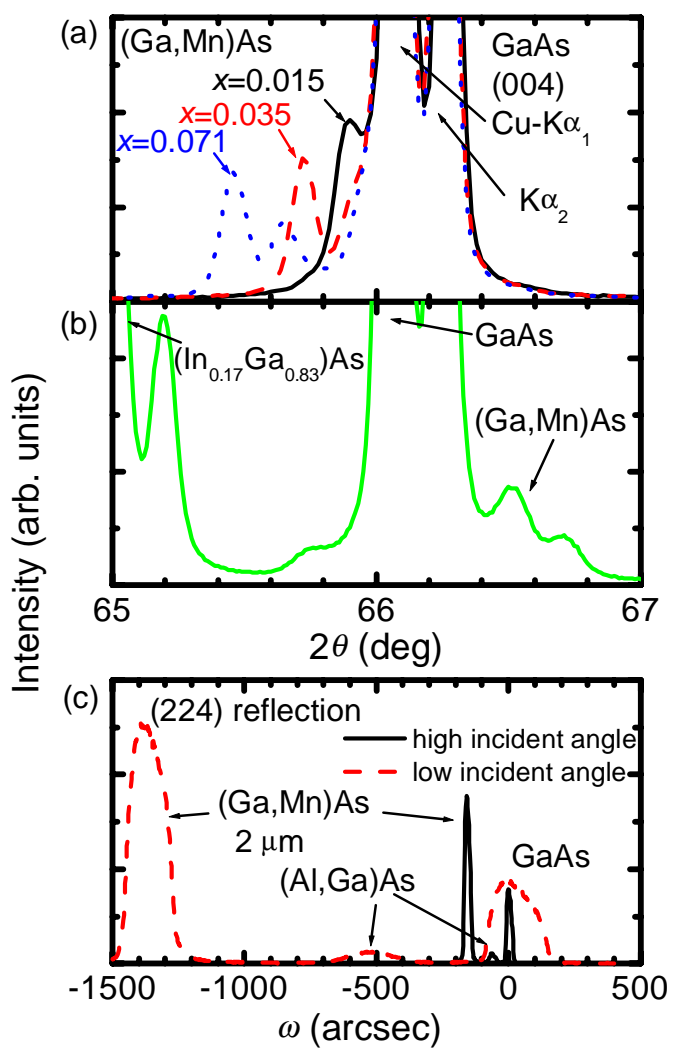

Fig. 7. X-ray diffraction curves for (Ga,Mn)As films obtained with $\mathrm{Cu} \mathrm{K} \alpha$ radiation. (a) $\mathrm{Mn}$ concentration dependence of peak positions [(004) reflection] of 150-nm thick (Ga,Mn)As grown on GaAs with compressive strain (Ohno et al. 1996a). (b) $(\mathrm{Ga}, \mathrm{Mn})$ As grown on $(\mathrm{In}, \mathrm{Ga})$ As buffer layer with tensile strain. (c) Double-crystal x-ray diffraction curves for a 2 mm-thick (Ga,Mn)As showing the asymmetric (224) reflection with high- and low-incident angle (Shen et al. 1999). 


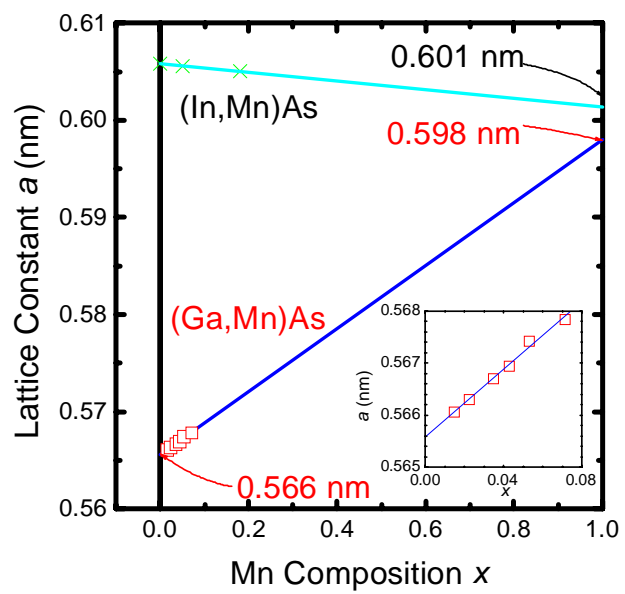

Fig. 8. Cubic lattice constant $a_{\mathrm{o}}$ versus Mn composition $x$ in $\mathrm{Ga}_{1-x} \mathrm{Mn}_{x}$ As and $\mathrm{In}_{1-\mathrm{x}} \mathrm{Mn}_{\mathrm{x}}$ As films. Inset shows the magnified view of the results for $(\mathrm{Ga}, \mathrm{Mn})$ As films (Munekata et al. 1999, Ohno et al. 1996a).

to $(\mathrm{Ga}, \mathrm{Mn})$ As on $(\mathrm{In}, \mathrm{Ga}) \mathrm{As}$ is located at the higher angle side of GaAs, indicating that the film is now under a tensile strain. In order to calculate the relaxed lattice constant $a_{0}$, it is assumed that elastic constants of $(\mathrm{Ga}, \mathrm{Mn}) \mathrm{As}$ are the same as in GaAs (Poisson ratio: $v=0.311$ ). On the other hand, since thick (In,Mn)As films on GaAs are fully relaxed, $a_{0}$ of (In,Mn)As can be directly determined from the positions of the diffraction peaks.

The dependencies $a_{0}(x)$ for $(\mathrm{Ga}, \mathrm{Mn}) \mathrm{As}$ and (In,Mn)As, as determined by XRD, are summarized in Fig. 8. In both materials, $a_{\mathrm{o}}$ depends linearly on $x$ following Vegard's law, which for $(\mathrm{Ga}, \mathrm{Mn}) \mathrm{As}$ assumes the form $a_{0}=0.566(1-x)+$ $0.598 x(\mathrm{~nm})$ (Ohno et al. 1996). The lattice constant is known to depend on the growth conditions such as As pressure and/or growth temperature due to the corresponding excess of As (Shimizu et al. 1999, Haneda et al. 2000a). A growth of (Ga,Mn)As under other conditions gives $a_{0}=0.5654(1-x)+$ $0.5901 x(\mathrm{~nm})$ (Sadowski et al. 2001a). It is shown that the lattice constant of $(\mathrm{Ga}, \mathrm{Mn})$ As depends on the growth condition, probably due to the excess As incorporation and the formation of a Mn-As complex (Schott et al. 2001). The extrapolated values of $a_{\mathrm{o}}(x)$ for $x \rightarrow 0$ are in good agreement with the actual GaAs and InAs lattice constants, respectively. The extrapolated lattice constants for hypothetical zinc-blende MnAs determined from (Ga,Mn)As and (In,Mn)As data show a good correspondence. This suggests that virtually all $\mathrm{Mn}$ atoms occupy the substitutional sites. The lattice constant of hypothetical zincblende MnAs in the ferromagnetic state has been predicted to be $0.59 \mathrm{~nm}$ by first-principle calculations (Shirai et al. 1998, Ogawa et al. 1999).

\subsection{Local lattice configuration (EXAFS)}

An extended x-ray absorption fine structure (EXAFS) study, carried out using the Mn K-edge, of thick ( $\geq 1$ $\mu \mathrm{m})(\mathrm{In}, \mathrm{Mn})$ As reveals that $\mathrm{Mn}$ is incorporated substitutionally into the In sites. This is especially true for low $x(\sim 0.01)$ samples grown at low $T_{\mathrm{S}}\left(200^{\circ} \mathrm{C}\right)$. Although the substitutional signal is still dominant, either increasing of $x$ above 0.1 or raising $T_{\mathrm{S}}$ to $300^{\circ} \mathrm{C}$ results in a modification of the local structure. In the case of greater $x$ or higher $T_{\mathrm{S}}$, the local structure shows that $\mathrm{Mn}$ is incorporated in the form of hexagonal MnAs (NiAs-structure) (Krol et al. 1993, Soo et al. 1996).

Fluorescence EXAFS studies of a (In,Mn)As thin layer $(10 \mathrm{~nm})$ grown on a GaSb buffer layer and of (In,Mn)As quantum dots (QDs) on GaAs were also performed. The results show that in the thin (In,Mn)As layer, the In-site substitutional $\mathrm{Mn}$ and the NiAs-type MnAs coexist, whereas the majority of $\mathrm{Mn}$ atoms are substituted in the In-sites of InAs in (In,Mn)As QDs. It is argued that the difference of the strain deformation between the thin layer (with strain) and thick layer and QDs (strain relaxed) is responsible for the differences in the local structure of the Mn atoms (Ofuchi et al. 2001b).

EXAFS measurements of (Ga,Mn)As $(x=0.005$ and 0.074) also indicate that $\mathrm{Mn}$ atoms are substitutionally incorporated into the Ga sublattice (Shioda et al. 1998). The Mn-As bond length is found to be $0.249-0.250 \mathrm{~nm}$, longer than the host Ga-As bond length $(0.244 \mathrm{~nm})$ and shorter than the expected bond length of Mn-As in the hypothetical zinc-blende MnAs (0.259 nm).

3.3. Atomic-scale observations by scanning tunneling microscopy (STM)

The cross-sectional scanning tunneling microscopy (XSTM) measurements were carried out for a cleaved (110) surface of GaAs doped with Mn. The samples were cleaved in an ultrahigh vacuum (UHV) chamber in order to expose the atomically flat (110) surface. The measurements have been done in the UHV chamber at room temperature.

The defects and impurities of Mn-doped GaAs (Mn composition $<0.001$ ) layers grown at $400^{\circ} \mathrm{C}$ are identified in the XSTM images. The results show that Mn impurities have negative charge, indicating that $\mathrm{Mn}$ acts as an acceptor. The $\mathrm{Mn}$ acceptor concentration deduced from the XSTM images is in a good agreement with the hole concentration determined by Hall measurements at room temperature. As-vacancy defects are also observed. Since the number of the vacancies increases with time, they are presumably formed after the cleavage due to the desorption of As atoms from the surface. No As-antisite related defects are detected in the samples grown at $400^{\circ} \mathrm{C}$ (Tsuruoka et al. 2000, 2002).

XSTM images for Mn-doped GaAs (Mn composition $=0.005$ ) grown at $255^{\circ} \mathrm{C}$ show that there are numerous As-antisites, the concentration of which is $\sim 1 \times 10^{20}$ $\mathrm{cm}^{-3}$, similar to LT-GaAs. The Mn concentration determined from the images is $7 \times 10^{19} \mathrm{~cm}^{-3}$, which is consistent with the nominal Mn composition $\sim 1 \times 10^{20} \mathrm{~cm}^{-3}$. The tunneling spectrum reveals the presence of a state in the midgap region caused by the As-antisite donors and a shift of the Fermi energy brought about by the incorporation of $\mathrm{Mn}$ because of its acceptor nature (Grandidier et al. 2000).

\section{Spin and charge states of Mn in III-V magnetic semiconductors}

Various properties of Mn impurity centers have been investigated by many methods, such as magnetic resonance and magnetization measurements, for a long time. It is expected that there are three possible electronic states of the Mn impurity 
substituting a trivalent cation: $A^{0}\left(\mathrm{~d}^{4}\right)$ and $A^{0}\left(\mathrm{~d}^{5}+\mathrm{h}\right)$ for $\mathrm{Mn}^{3+}$, and $A^{-}\left(\mathrm{d}^{5}\right)$ for $\mathrm{Mn}^{2+} \cdot A^{0}$ denotes the neutral center, $A^{-}$is the negatively charged center, and the notation in parentheses is the electronic configuration of the d electrons. There have been no reports on the observation of $A^{0}\left(\mathrm{~d}^{4}\right)$ neutral centers in GaAs. In contrast, the anisotropy of some of electron spin resonance (ESR) lines in the illuminated n-type GaP:Mn can be explained in terms of $A^{0}\left(\mathrm{~d}^{4}\right)$ centers (Kreisel et al. 1996) that undergo a Jahn-Teller distortion, as observed for $\mathrm{Cr}\left(3 \mathrm{~d}^{4}\right)$ in GaAs (Krebs and Stauss 1977).

In the case of the $A^{0}\left(\mathrm{~d}^{4}\right)$ center the hole resides in the $3 \mathrm{~d}$ shell. However, strong Hund's intra-site exchange interaction may favor a state having five $\mathrm{d}$ electrons and a loosely bound hole. This is the case of the $A^{0}\left(\mathrm{~d}^{5}+\mathrm{h}\right)$ configuration, where the $A^{0}\left(\mathrm{~d}^{4}\right)$ center traps tightly an electron in the $3 \mathrm{~d}$ shell forming the high spin, $S=5 / 2,3 \mathrm{~d}^{5}$ configuration, and this negatively charged $\mathrm{Mn}$ ion binds the hole in an effective mass state. Experimental results discussed below indicate that the ground state of the $\mathrm{Mn}$ impurity in III-V compounds corresponds to such $A^{0}\left(\mathrm{~d}^{5}+\mathrm{h}\right)$ configuration.

\subsection{Electron spin resonance (ESR)}

The result of ESR measurements for bulk Mn doped GaAs (GaAs:Mn) with Mn concentration of $\sim 1 \times 10^{17} \mathrm{~cm}^{-3}$ is interpreted in terms of the $A^{0}\left(\mathrm{~d}^{5}+\mathrm{h}\right)$ acceptor state. Owing to the antiferromagnetic exchange coupling between the d-electrons and the p-like hole, the total angular momentum is $J=1$ (Schneider et al. 1987, Masterov et al. 1988). The ESR spectra for bulk GaAs:Mn with a Mn concentration of $10^{17} \sim 10^{18} \mathrm{~cm}^{-3}$ show resonance with unresolved hyperfine structure even at $4 \mathrm{~K}$, which can be attributed to the existence of $A^{-}\left(\mathrm{d}^{5}\right)$ centers (Almelsh and Goldstein 1962). Results of low-temperature magnetization measurements on GaAs:Mn with a hole concentration of $5 \times 10^{18} \mathrm{~cm}^{-3}$ at $300 \mathrm{~K}$ are consistent with the coexistence of $A^{0}\left(\mathrm{~d}^{5}+\mathrm{h}\right)$ and $A^{-}\left(\mathrm{d}^{5}\right)$ centers (Mac et al. 1994).

Electron spin resonance (ESR) spectra for (Ga,Mn)As grown by LT-MBE show usually only one resonance corresponding to $g=2.0$ (Nojiri et al. 1998, Szczytko et al. 1999a). This resonance can be attributed to $A^{-}\left(\mathrm{d}^{5}\right)$ centers. The ESR intensity becomes weak around the Curie temperature; i.e., the observed signal is due to the ferromagnetic resonance (FMR). The description of the observed signal in terms of the standard FMR formula, in which the magnetic crystalline anisotropy is neglected, indicates that the magnitude of magnetization is smaller than that expected for the given $x$ value This suggests that only a part of the Mn spins contributes to the ferromagnetic order in (Ga,Mn)As. The conclusions consistent with results of other studies that will be discussed below. From the fine structure of the ESR spectrum of $(\mathrm{Ga}, \mathrm{Mn})$ As with $x<$ $1.5 \times 10^{-3}$, the single-ion crystal-field anisotropy of the Mn spin energy was determined (Fedorych et al. 2001).

No signal of $A^{0}\left(\mathrm{~d}^{5}+\mathrm{h}\right)$ centers is usually detected in (Ga,Mn)As grown by LT-MBE (Nojiri et al. 1998, Szczytko et al. 1999a). The reason can be either the compensation by the antisite donors in the low $x$ limit or the high hole concentration for larger $x$ values, which leads to screening of the Coulomb potentials of the $A^{-}\left(\mathrm{d}^{5}\right)$ centers, resulting in a low ionization energy of the holes and eventually to the insulator-to-metal transition. A similar situation occurs in the case of (In,Mn)As layers (Szczytko et al. 2001a). A negligible contribution of the centers other than $A^{-}\left(\mathrm{d}^{5}\right)$ suggests that the double-exchange mechanism of the coupling between the Mn spins is ineffective, as this mechanism requires the coexistence of the $\mathrm{Mn}$ ions with a different valence.

\subsection{Optical spectroscopy}

The results of infrared absorption measurements on GaAs:Mn prepared by the solid-state diffusion method are also in good agreement with the $A^{0}\left(\mathrm{~d}^{5}+\mathrm{h}\right)$ center model (Linnarsson et al. 1997). According to infrared spectroscopy and photoluminescence (PL) measurements for GaAs:Mn with a Mn concentration of $\sim 10^{18} \mathrm{~cm}^{-3}$, this acceptor level is located 113 $\mathrm{meV}$ above the top of the valence band (Chapman and Hutchinson 1967, Ilegems et al. 1975). Two photoluminescence (PL) lines observed by Liu et al. (1995) in magnetic fields up to $30 \mathrm{~T}$ were identified as radiative recombination of the hole bound to $\mathrm{Mn}$ with conduction band and donor electrons, respectively. From the field-induced PL line splittings, an effective Landé factor of the neutral acceptor $g=2.47$ was determined, confirming qualitatively the ESR results discussed above. This work corroborated also an earlier conclusion (Schairer and Schmidt 1974) about the absence of transitions involving excitons bound to neutral Mn acceptors in GaAs:Mn. This surprising result was explained by Bhattacharjee and Benoit a la Guillaume (2000) taking into account the presence of a strong exchange interaction between the holes and $\mathrm{Mn}$ ions. In contrast, Sapega et al. (2001) assigned a weak line in their PL spectrum to such a transition. At the same time, this transition energy corresponds to a resonant enhancement of spin-flip Raman scattering studied in detail by Sapega et al. (2001). However, to interpret their Raman spectra, a coupling of bound excitons to more than one $\mathrm{Mn}$ ion had to be invoked by Sapega et al. (2001). It appears probable, therefore, that the PL line and spin-flip Raman scattering in question originate from complexes involving the hole interacting with a pair of nearest neighbor $\mathrm{Mn}$ ions or with other types of Mn clusters. Isolated neutral acceptors, in turn, being unable to bind any exciton, are invisible in spin-flip Raman spectroscopy. This would explain why the p-d exchange energy determined by Sapega et al. (2001) is much smaller than those imply by other experiments.

\subsection{X-ray magnetic circular dichroism (XMCD)}

X-ray absorption spectroscopy (XAS) provides direct information of the $3 \mathrm{~d}$ electronic structure of $\mathrm{Mn}$, since the $2 p$ electron is ensured to be excited into $3 d$ state because of the dipole selection rule. The x-ray magnetic-circular dichroism (XMCD) spectrum obtained for 150-nm thick (Ga,Mn)As with $x$ $=0.02$ in the photon energy region $630-660 \mathrm{eV}$ shows the two groups of rich peak structures associated with $2 p_{3 / 2}$ and $2 p_{1 / 2}$ final state holes. The rich structures are caused by the spin-orbit interaction of the holes and the Coulomb and exchange interactions between the $2 p$ core and $3 d$ shell (Ohldag et al. 2000). The temperature dependence of $\mathrm{XMCD}$ at $642.2 \mathrm{eV}$, at which the $2 p_{3 / 2}$-related signal has maximum intensity, can be well fit by a mean-field model with $T_{\mathrm{C}}$ of $37 \mathrm{~K}$, which is consistent with $T_{\mathrm{C}}$ of (Ga,Mn)As with $x=0.02$. Comparison of measured and calculated XMCD spectra shows that a linear 
combination of $\mathrm{Mn} 3 \mathrm{~d}^{5} \quad(80 \%)$ and $\mathrm{Mn} 3 \mathrm{~d}^{6} \quad(20 \%)$ configurations gives the best agreement. This configuration appears to occur for all $\mathrm{Mn}$ atoms whose spins are, therefore, highly localized and the magnetic moment is about $4.5 \mu_{\mathrm{B}}$. From the ratio of the observed MCD to the calculated MCD, only $13 \%$ of $\mathrm{Mn}$ in $(\mathrm{Ga}, \mathrm{Mn})$ As contributes to the ferromagnetic order. This is consistent with the magnetization measurement for (Ga,Mn)As with $x=0.023$, where considerable amounts of Mn spins behave as paramagnetic spins (Oiwa et al. 1998a). For as-grown samples, XAS shows two-component contributions of $\mathrm{Mn}$ to the spectrum. Since one of them disappears after low-temperature heat-treatment $\left(<300^{\circ} \mathrm{C}\right)$, it may be related to Mn-As complexes (Katsumoto et al. 2001). The analysis of XAS and XMCD based on the cluster model shows that the orbital momentum of Mn electrons is small and that the sign of the p-d interaction is antiferromagnetic (Ueda et al. 2001). The result shows that the $3 \mathrm{~d}$ electron count of $\mathrm{Mn}$ is $\sim 5\left(A^{0}\left(\mathrm{~d}^{5}+\mathrm{h}\right)\right.$ or $\left.A^{-}\left(\mathrm{d}^{5}\right)\right)$, which is consistent with the lack of the $A^{0}\left(\mathrm{~d}^{4}\right)$ centers in the ESR signals.

\subsection{Photoemission}

The core-level x-ray photoemission spectrum of the Mn 2p core level for (Ga,Mn)As with $x=0.074$ was measured and was analyzed by configuration interaction (CI) cluster-model assuming a $\mathrm{Mn}^{2+}$ and $\mathrm{Mn}^{3+}$ ground state (Okabayashi et al. 1998). For the $d^{5}$ configuration, the $\mathrm{p}-\mathrm{d}$ exchange energy (which is conventionally referred to as $N_{0} \beta$ for DMS) should be negative and $N_{0} \beta \sim-1.2 \mathrm{eV}$ is obtained for $A^{-}\left(d^{5}\right)$ centers with an optimized parameter set.

The resonant photoemission technique was used for several (Ga,Mn)As layers to elucidate the nature of the Mn 3d partial density of states (DOS) (Okabayashi et al. 1999, 2001a, 2001b). The main structure of Mn partial DOS extends from the Fermi energy $E_{\mathrm{F}}$ down to $-4.5 \mathrm{eV}$ below it. The configuration interaction (CI) model calculation indicates an enhanced Mn 3d electron count of 5.3. The largest contribution to the DOS at $E_{\mathrm{F}}$ comes from As $4 p$ states. However, the Mn partial DOS extends up to the top of the valence band, suggesting partial $d$ character of the conducting holes. On the other hand, in-situ measurement on an as-grown sample shows a suppression of the $d$ character of the holes at Fermi level, suggesting that the observed d-character of the conducting holes may be related to the experimental error due to the surface condition (Okabayashi et al. 2001c). In the region extending from the Fermi energy $E_{\mathrm{F}}$ down to $0.5 \mathrm{eV}$ below it, an increase in emission intensity due to Mn-induced states is observed in $\mathrm{Ga}_{1-x} \mathrm{Mn}_{x} \mathrm{As}$ with $x=0.035$ and 0.069 (Okabayashi et al. 2001a, 2001b). Since an insulator-to-metal transition occurs in the vicinity of this $\mathrm{Mn}$ concentration (Oiwa et al. 1997, Matsukura et al. 1998b), it is tempting to assign this DOS to Mn acceptor states merging with the valence band. No clear Fermi edge is observed, which may be due to a relatively low hole concentration and poor metallic conduction.

\section{Magnetic properties}

\subsection{Magnetization}

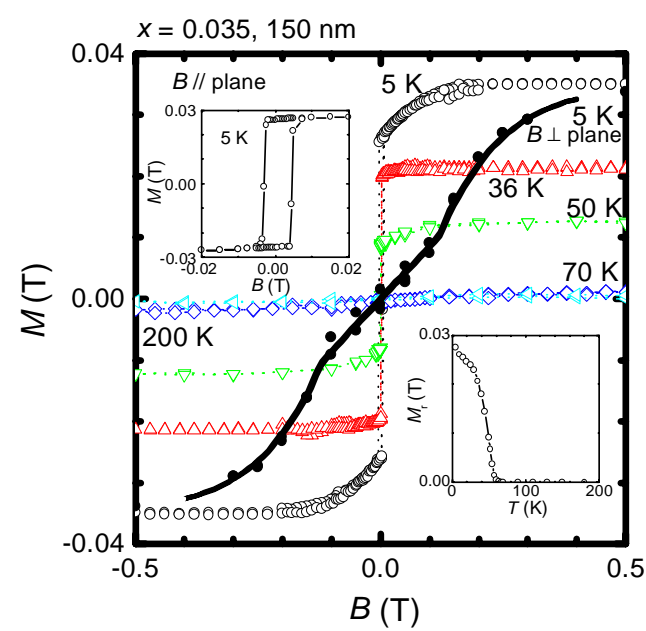

Fig. 9. Magnetic field dependence of the magnetization at selected temperatures for a 150 -nm thick $\mathrm{Ga}_{1-x} \mathrm{Mn}_{x}$ As film with a Mn composition $x=0.035$. The magnetic field is applied parallel to the sample surface (direction of magnetic easy axis) except for the closed circles at $5 \mathrm{~K}$ taken in perpendicular geometry. The solid line for $5 \mathrm{~K}$ shows the magnetization determined from transport measurements. The upper left inset shows a magnified view of the magnetization in the parallel field at $5 \mathrm{~K}$. The lower right inset shows the temperature dependence of the remanent magnetization. (Ohno et al. 1996a).

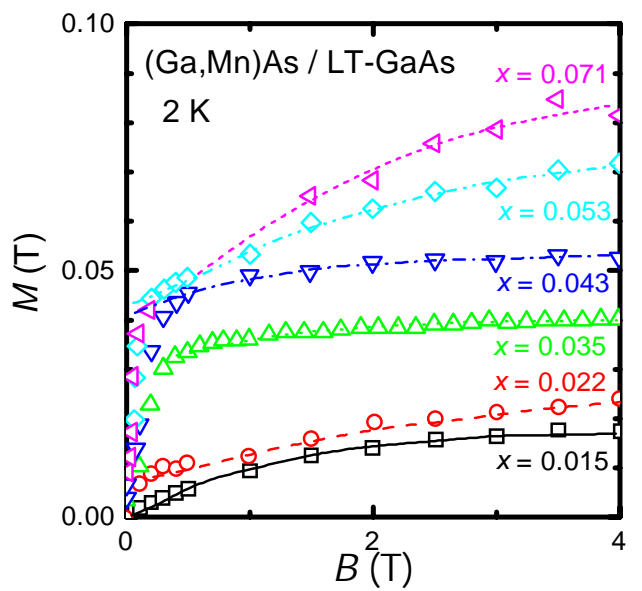

Fig. 10. Magnetization for six samples of $\mathrm{Ga}_{1-x} \mathrm{Mn}_{x} \mathrm{As} / \mathrm{GaAs}$ with Mn compositions $x$ ranging from 0.015 to 0.071 at $2 \mathrm{~K}$. The magnetic field is perpendicular to the sample surface for $x$ $=0.035 \sim 0.071$ and parallel to it for $x=0.015$ and 0.022 . The dashed lines show fit to the mean-field Brillouin function and the solid line for $x=0.015$ (paramagnetic sample) a fit to the Brillouin function (Oiwa et al. 1998a).

Magnetic properties of III-V DMSs can be measured by direct magnetization measurements as well as magnetotransport measurements. In this chapter, we focus on the magnetic properties of $(\mathrm{Ga}, \mathrm{Mn}) \mathrm{As}$ obtained by direct magnetization measurements. For (In,Mn)As, since there are only limited magnetization measurements, we will describe magnetic and magnetotransport properties together in the next chapter.

Direct measurements of the magnetization $M$ of (Ga,Mn)As layers as a function of magnetic field $B$ and temperature $T$ have been done using a commercially available superconducting quantum interference device (SQUID) magnetometer. The temperature-independent diamagnetic response of the thick GaAs substrate (which could occasionally show a slight temperature dependence of unknown origin) can 


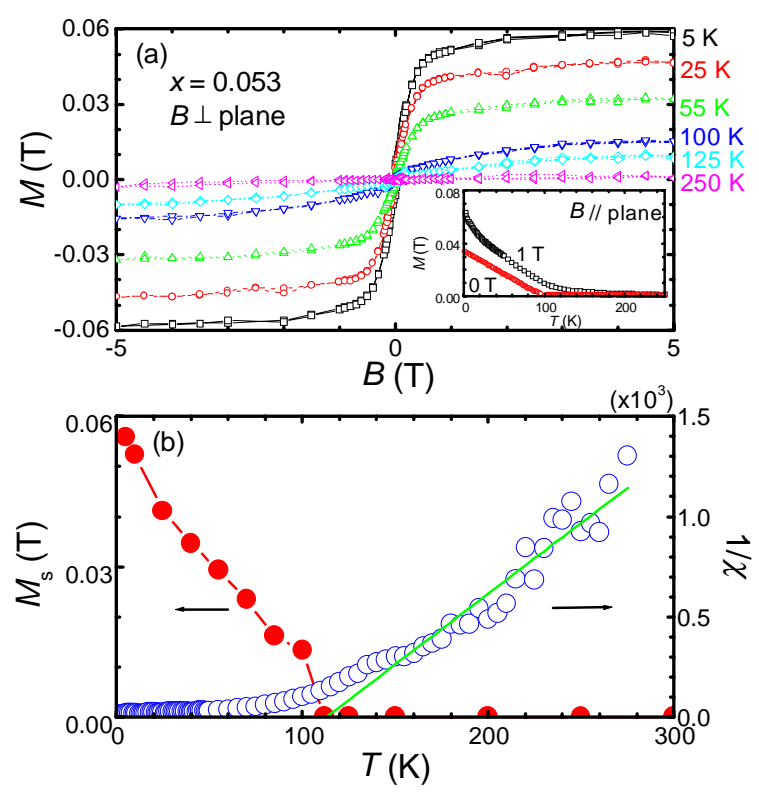

Fig. 11. (a) Temperature dependence of the magnetization for 200-nm thick $\mathrm{Ga}_{1-x} \mathrm{Mn}_{x}$ As with $x=0.053$. The magnetic field is applied perpendicular to the sample surface (hard axis). The inset shows temperature dependence of the remanent magnetization $(0 \mathrm{~T})$ and the magnetization at $1 \mathrm{~T}$ in a field parallel to the film surface. (b) Temperature dependence of the saturation magnetization $M_{\mathrm{S}}$ determined from the data shown in (a) by using Arrott plots (closed circles). Open circles show the inverse magnetic susceptibility and the Curie-Weiss fit is depicted by the solid straight line. (Ohno and Matsukura 2001).

be determined from a separate measurement of only the same GaAs substrate used for the epitaxial growth. It can also be determined from the low-temperature high-filed magnetization measurements, where the magnetization of the (Ga,Mn)As layer should saturate, or from high-temperature measurements, where the magnetization of the (Ga,Mn)As of the epitaxial layer on the substrate should be negligible. The diamagnetic component is then subtracted from the total response to obtain the magnetization of the magnetic layer.

Figure 9 shows magnetization curves at several temperatures of a 150 -nm thick $\left(\mathrm{Ga}_{0.965} \mathrm{Mn}_{0.035}\right)$ As layer grown on GaAs, where $B$ is applied parallel and perpendicular to the plane. As shown in the inset, when $B$ is applied parallel to the plane, the $M-B$ curve shows a clear hysteresis as in the upper right inset, which indicates the presence of a ferromagnetic order. A paramagnetic response is often observed after closure of the hysteresis in the magnetization curves, as seen in the $5 \mathrm{~K}$ curve of Fig. 9 and shown for (Ga,Mn)As with several Mn compositions in Fig. 10. This paramagnetic response is correlated with the transport properties of the film, the more metallic sample (in terms of the metal-insulator transition (MIT)) the less the portion of paramagnetic response (Oiwa et al 1998a, 1999).

Shown in the lower left inset of Fig. 9 is the temperature dependence of remanence of $M$ after switch off of parallel $B$, which reveals that the $T_{\mathrm{C}}$ of the film is $\sim 60 \mathrm{~K}$. Note also that there is no indication of inclusion of MnAs with NiAs-structure $\left(T_{\mathrm{C}} \sim 310 \mathrm{~K}\right)$ in the trace, as only a negligible $M$ is present above the $T_{\mathrm{C}}$ of $(\mathrm{Ga}, \mathrm{Mn}) \mathrm{As}$. This, however, is not always the case, and a small non-zero $M$ up to $300 \mathrm{~K}$ is occasionally observed, especially in samples grown at higher $T_{\mathrm{S}}$ or having large $x$.
The solid line in Fig. 9 shows $M$ determined by the transport measurements, where the Hall resistance is almost proportional to the perpendicular component of $M$, as described in the next section. The good agreement between $M$ determined by SQUID and transport measurements indicates that one can correctly determine $M$ of $(\mathrm{Ga}, \mathrm{Mn})$ As by magnetotransport measurements.

The saturation magnetization $M_{\mathrm{Sat}}(M$ at $T=5 \mathrm{~K}$ and $B>5 \mathrm{~T})$ of the samples shown in Figs. 9-11 indicates that $S=$ 2.0 2.5 when calculated using $M_{\text {Sat }}=x N_{0} g \mu_{\mathrm{B}} S$ and nominal $x$ as well as neglecting the hole contribution (Dietl et al. 2001c). Although $S$ is related to the valence of $\mathrm{Mn}$ and thus to the mechanism of ferromagnetism, a more accurate determination of $S$ from magnetization measurements is not possible because of the error involved in the determination of the $x$ value $( \pm$ $10 \%)$.

Figure 11 (a) presents magnetization determined for a 200-nm thick film of $\mathrm{Ga}_{0.947} \mathrm{Mn}_{0.053}$ As. Here almost no hysteresis is observed because $B$ is applied perpendicular to the sample plane along the magnetic hard axis. The inset displays the temperature dependence of the remanent magnetization for $B / /$ plane, showing that $T_{\mathrm{C}}$ is above $100 \mathrm{~K}$. Using Arrott plots to minimize the effect of magnetic anisotropy and domain rotation (Arrott 1957), the spontaneous magnetization $M_{\mathrm{S}}$ at each temperature is determined from the curves in Fig. 11 (a) and plotted in Fig. 11 (b). The $T_{\mathrm{C}}$ of this sample is about $110 \mathrm{~K}$. The non-standard temperature dependence of $M_{\mathrm{S}}$ shows a rather steep increase at low $T$. The temperature dependence of the inverse susceptibility $1 / \chi$ shown in the same figure gives a paramagnetic transition temperature $\theta$ consistent with the $T_{\mathrm{C}}$ determined from $M_{\mathrm{S}}$. For the extrapolation of $1 / \chi$, data points above $150 \mathrm{~K}$ were used.

The Curie constant $C=g^{2} \mu_{\mathrm{B}}^{2} x N_{0} S(S+1) / 3 k_{\mathrm{B}} T$, determined from $1 / \chi$ - $T$ curve shown in Fig. 11 (b) gives a spin of $S \cong 3$, when the nominal value of the Mn concentration, $x=$ 0.053 , is used. Here $N_{0}$ is the cation density, $g(=2.0)$ the Landé factor of the Mn ions, $\mu_{\mathrm{B}}$ is the Bohr magneton, $k_{\mathrm{B}}$ is the Boltzmann constant. Judging from this value of $S$, some ferromagnetic clusters exist already above $T_{\mathrm{C}}$.Magnetization measurements on semi-insulating $(\mathrm{Ga}, \mathrm{Mn}) \mathrm{As}: \mathrm{Sn}$, in which the holes are compensated by Sn donors, point to a paramagnetic behavior. The Curie-Weiss $(1 / \chi-T)$ plot gives $T_{\mathrm{AF}} \sim 2 \mathrm{~K}$, indicating that the "intrinsic" mechanism of the interaction between the Mn spins is the antiferromagnetic superexchange (Satoh et al. 1997, 2001). This means that the ferromagnetic coupling in $(\mathrm{Ga}, \mathrm{Mn})$ As is induced by the presence of the holes.

\subsection{Magnetic anisotropy}

As shown in Fig. 9 for the magnetization at $5 \mathrm{~K}$, the magnetic easy axis is in the plane of the film, and shows a weak four-fold symmetry within the plane. The anisotropy energy $K$ calculated from the difference between the two magnetization curves at $5 \mathrm{~K}$ with $B \perp$ plane and $B / /$ plane is $K=2.9 \times 10^{3} \mathrm{~J} / \mathrm{m}^{3}$. This anisotropy energy of (Ga,Mn)As is strain dependent (Ohno et al. 1996b, Shen et al. 1997a) and is also expected to depend on the hole concentration (Abolfath et al. 2001, Dietl et al. 2001c). The strain in the film shown in Fig. 9 is $-0.24 \%$. The magnetic easy axis can be made perpendicular to the plane by the reversing the sign of strain in the film. 


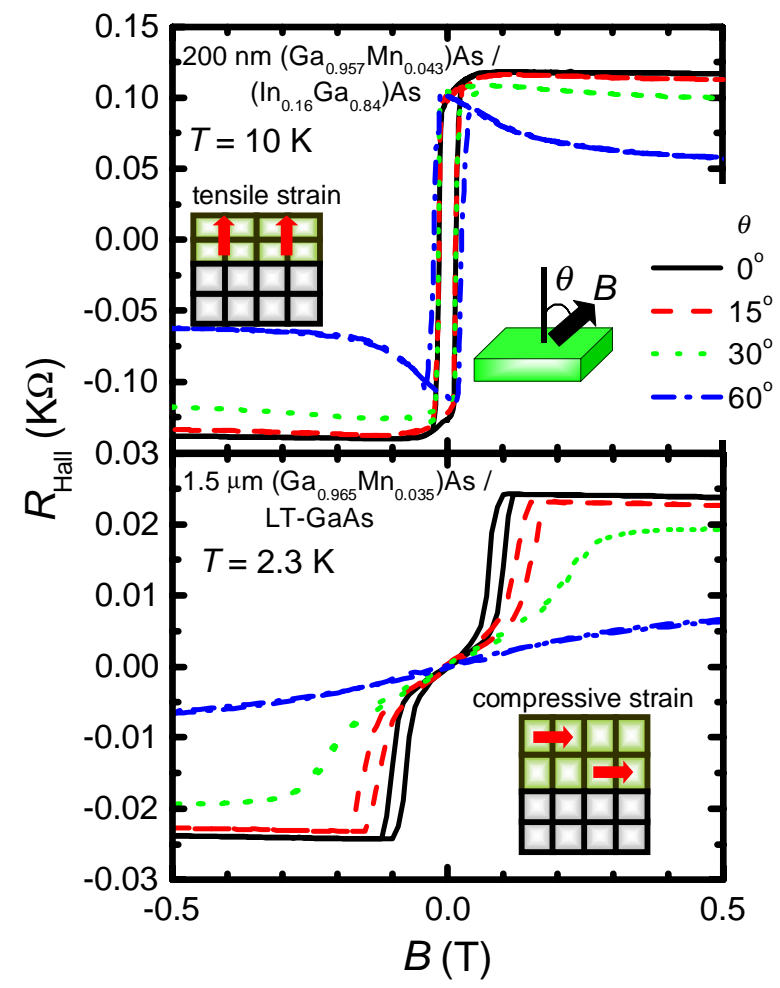

Fig. 12. Hall resistance $R_{\text {Hall }}$ of (a) $(\mathrm{Ga}, \mathrm{Mn}) \mathrm{As} /(\mathrm{In}, \mathrm{Ga}) \mathrm{As}$ and (b) $(\mathrm{Ga}, \mathrm{Mn}) \mathrm{As} / \mathrm{GaAs}$ as a function of the magnetic field for various angles between the field and the sample surface normal. (Ga,Mn)As films in (a) and (b) are under tensile and compressive strain, respectively. Clear hysteresis and angle independent heights of the hysteresis in (a) show that magnetic easy axis is perpendicular to the sample surface, whereas the easy axis in (b) is in-plane. (Shen et al. 1997a).

Figure 12 shows clearly the different directions of the magnetic easy axis for the different buffer layers. Figure 12 (a) shows the direction of the $B$ dependence of the Hall resistance $R_{\text {Hall }}$ for $(\mathrm{Ga}, \mathrm{Mn})$ As with tensile strain on a thick lattice-relaxed $\left(\mathrm{In}_{0.16} \mathrm{Ga}_{0.84}\right)$ As buffer layer and Fig. 12 (b) for $(\mathrm{Ga}, \mathrm{Mn})$ As with compressive strain on a GaAs buffer layer. Only the (Ga,Mn)As with tensile strain shows a clear hysteresis which suggests a change of the direction of the magnetic easy axis. The height of the hysteresis is almost independent of the direction of the magnetic field, which shows that the direction of the easy axis is perpendicular to the plane. On the other hand, $(\mathrm{Ga}, \mathrm{Mn})$ As with compressive strain shows only a small hysteresis and no perpendicular component of the remanent magnetization. The result confirms that the direction of the magnetic easy axis of (Ga,Mn)As with compressive strain is in-plane (Ohno et al. 1996b).

The significant increase of coercive force and $T_{\mathrm{C}}$ of (Ga,Mn)As with spinglass $(\mathrm{Zn}, \mathrm{Mn}) \mathrm{Se}$ overlayer on it are observed. The observed effects are technological important to control the hardness of ferromagnetism. Although the origin of which is not clear yet, it is most probably related to the exchange coupling between (Ga,Mn)As and (Zn,Mn)Se (Liu, X. et al. 2001).

\subsection{Magnetic domains}

Using a scanning Hall microscope, a stripe-shaped domain structure has been observed in a $(\mathrm{Ga}, \mathrm{Mn})$ As sample with tensile strain and perpendicular easy axis (Shono et al.
2000, Fukumura et al. 2001). The Baukhausen noise due to the scattering from the domain wall movement has also been observed in magnetotransport measurements (Hayashi et al. 2000).

Ac-susceptibility measurements on (Ga,Mn)As with $x=0.042$ have been performed in alternating $B$ from $0.1 \sim 4 \mathrm{mT}$. In the temperature dependence of the susceptibility, there is a sharp peak at about $48 \mathrm{~K}$ at $0.1 \mathrm{mT}$, which suggests a ferromagnetic phase transition. The temperature and magnetic field dependence is rather complicated and an increase of $B$ involves additional peaks (the number of which up to 4), which may be due to domain formation and domain wall movement (Sadowski et al. 2000). There is also a report about ac-susceptibility measurements on (Ga,Mn)As with $x=0.07$, which shows that there is no difference between the field-cooled and zero-field cooled ac-susceptibilities measured with $B=10$ mT (Van Esch et al. 1997).

\subsection{Cantilever magnetometry}

Torsional magnetometry using submicron GaAs micromechanical cantilevers is useful for measuring the magnetic properties of small samples over a wide range of applied magnetic fields and temperatures. Since (Ga,Mn)As can be grown epitaxially on GaAs, it can be incorporated in a GaAs cantilever using photolithography. 15- $\mu \mathrm{m}$-radius disk-shape mesas of $\left(\mathrm{Ga}_{0.962} \mathrm{Mn}_{0.038}\right)$ As samples with $6 \mathrm{~nm}$ and $20 \mathrm{~nm}$ of thickness have been fabricated at the end of the cantilevers and their magnetic properties have been investigated (Harris et al. 1999). The cantilevers are $50 \mu \mathrm{m}$ wide and $100 \mathrm{~nm}$ thick and the longest lever is $\sim 400 \mu \mathrm{m}$ long. The cantilever with (Ga,Mn)As shows a twist of several degrees in the last $50 \mu \mathrm{m}$ due to the lattice mismatch between $\mathrm{GaAs}$ and $(\mathrm{Ga}, \mathrm{Mn})$ As. From the measurement, it is found that the torsional magnetometers have quite a high sensitivity of $3 \times 10^{6} \mu_{\mathrm{B}}$ at $0.1 \mathrm{~T}$. The obtained magnetization curves for 20-nm thick (Ga,Mn)As shows ferromagnetic behavior with an in-plane easiy axis, being consistent with the results obtained with a SQUID magnetometer for much larger samples. From the temperature dependence of the remanent moment, $T_{\mathrm{C}}$ is determined as $39 \mathrm{~K}$. Illumination of the sample with a blue light-emitting diode (LED) has no measurable effect on the magnetization of (Ga,Mn)As either above or below $T_{\mathrm{C}}$. Measurement on 6-nm thick (Ga,Mn)As shows no ferromagnetism down to $350 \mathrm{mK}$, which is consistent with SQUID results (Tanaka 1998).

\section{Magnetotransport properties}

Due to the presence of the anomalous Hall effect (known also as the extraordinary or spin Hall effect), magnetotransport measurements provide valuable information on the magnetism of thin films. The Hall resistance $R_{\text {Hall }}$ is empirically known to be a sum of ordinary and anomalous Hall terms,

$$
R_{\text {Hall }}=\frac{R_{0}}{d} B+\frac{R_{S}}{d} M_{\perp}
$$


Here, $R_{0}$ and $R_{\mathrm{S}}$ are the ordinary and anomalous Hall coefficients, respectively; $d$ is the thickness of the conducting channel; $M_{\perp}$ is the component of magnetization perpendicular to the sample surface (Chien and Westgate 1980), and $R_{\mathrm{S}}$ is proportional to $R_{\text {sheet }}{ }^{\gamma}$ with temperature-independent proportionality constant, where $R_{\text {sheet }}$ is the sheet resistance. Usually, $\gamma$ is either 1 or 2 depending on the origin of the effect; the skew-scattering mechanism results in $\gamma=1$, whereas for the side-jump mechanism $\gamma=2$. The proportionality constant, which determines the overall magnitude of the anomalous Hall effect, scales with the strength of the spin-orbit coupling for the carriers at the Fermi level as well as with the exchange energy describing the ratio of carrier spin polarization to magnetization $M_{\perp}$. Accordingly, at given $M_{\perp}$, the effect is expected to be much stronger for the holes than for the electrons in the tetrahedrally coordinated semiconductors. Furthermore, since its magnitude depends on the degree of spin polarization of the carrier liquid, it ceases to be proportional to the magnetization when the carrier spin-splitting becomes comparable to the Fermi energy.

Because of the high sensitivity, the determination of the magnetization by magnetotransport studies is the important technique for thin films of diluted magnets, in which the magnitude of the total magnetic moment is rather small. Accordingly, recent years have witnessed a renewed interest in the theory of the anomalous Hall effect (Hirsch 1999, Ye et al. 1999, Zhang 2000, Ferrand et al. 2000, Crépieux and Bruno 2001, Jungwirth et al. 2001). Theoretical calculations for p-type DMS, based on the side-jump mechanism, suggests an increase of the Hall conductivity with the decrease of the carrier concentration (Jungwirth et al. 2001). Experimental results discussed below: (i) demonstrate the critical importance of the Hall effect in the assessment of the magnetic properties of III-V ferromagnetic semiconductors; (ii) suggest that both side-jump and skew-scattering mechanisms operate (iii) point to various effects that can lead to differences between magnetizations determined by standard and Hall magnetometry. Furthermore, the accumulated magnetoresistance data indicate a significance of the spin-disorder scattering as well as reveal various effects associated with the interplay between spin and localization phenomena, specific to doped DMS in the vicinity of the metal-insulator transition (Dietl 1994).

\section{1. $(\mathrm{Ga}, \mathrm{Mn}) \mathrm{As}$}

\subsubsection{The Hall effect}

Figure 13 (a) presents the Hall resistance $R_{\text {Hall }}$ at various temperatures plotted as a function of the magnetic field for the same sample, for which magnetization data were collected in Fig. 11 (200-nm thick $\left.\mathrm{Ga}_{0.947} \mathrm{Mn}_{0.053} \mathrm{As}\right)$. The inset shows the temperature dependence of $R_{\text {sheet }}$. A close similarity between the results of Figs. 13 (a) and 11 (a) indicates that the contribution from the ordinary Hall term is rather small in the displayed field and temperature range. Thus, if skew-scattering is assumed, $R_{\text {Hall }} \approx c R_{\text {sheet }} M$, where $c$ is a temperature-independent constant. Actually, a comparison of magnetization and magnetotransport data suggests the value of the exponent $\gamma$ to be between 1 and 2 at low temperatures, and that the assumption of either mechanism leads to virtually identical conclusions about the magnetic properties. In
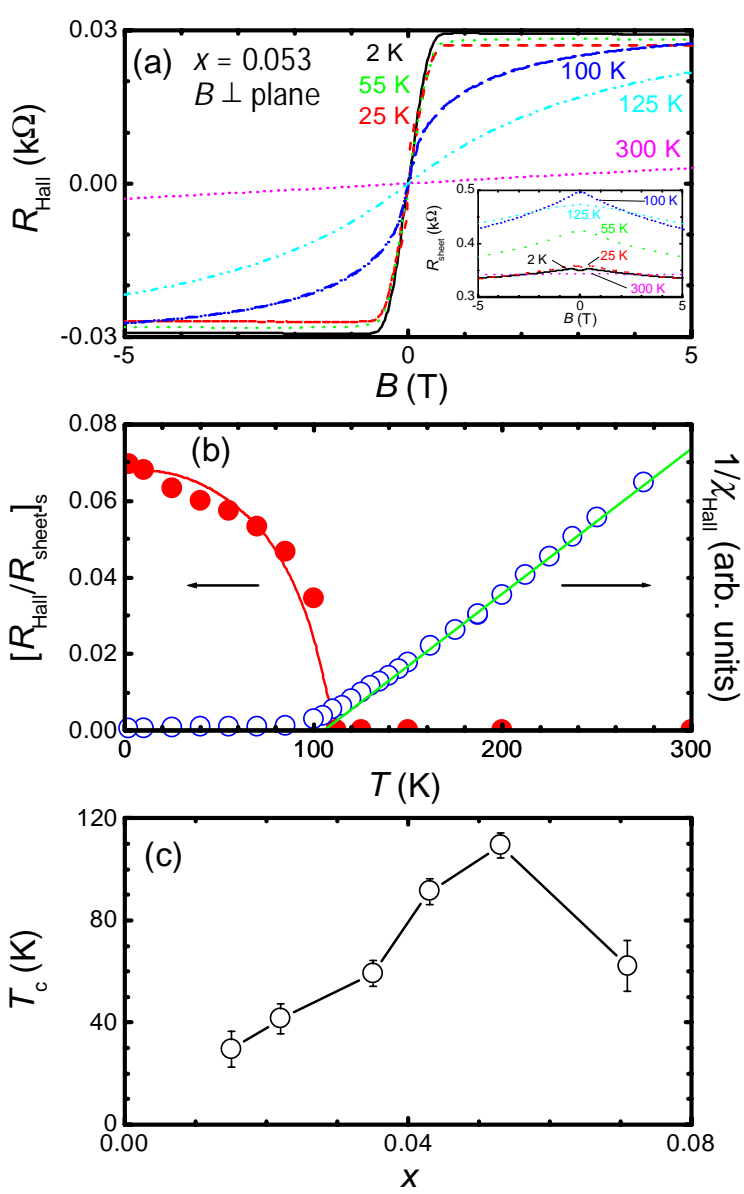

Fig. 13. (a) Temperature dependence of the Hall resistance $R_{\text {Hall }}$ for the same sample as in Fig. 11. The inset shows the temperature dependence of the sheet resistance $R_{\text {sheet. }}$ (b) Temperature dependence of the saturation magnetization $\left[R_{\text {Hall }} / R_{\text {sheet }}\right]_{\mathrm{S}}$ obtained using Arrott plots (closed circles) and inverse susceptibility $1 / \chi_{\text {Hall }}$ (open circles), both from the transport data shown in (a). Solid lines depict $\left[R_{\text {Hall }} / R_{\text {sheet }}\right]_{\mathrm{S}}$ and $1 / \chi_{\text {Hall }}$ calculated assuming the mean-field Brillouin behavior for the $\mathrm{Mn}$ spin $S=5 / 2$ and the Curie-Weiss law, respectively. (c) Mn composition dependence of the magnetic transition temperature $T_{\mathrm{C}}$, as determined from transport data. (Ohno and Matsukura 2001).

particular, since $R_{\text {Hall }} / R_{\text {sheet }} \propto M$, Arrott's plots can be employed to determine the temperature dependence of spontaneous magnetization $M_{\mathrm{S}}$.

The outcome of transport measurements is summarized in Figs. 13 (b) and 13(c). The values of $T_{\mathrm{C}}$ are in good agreement with those determined from the direct magnetization measurements. As shown in Fig. 13 (c), the value of the Curie temperature is almost proportional to $x$ up to $x$ of about $5 \%$ according to $T_{\mathrm{C}} \approx 2000 x \pm 10 \mathrm{~K}$. However, a further increase of $x$ decreases $T_{\mathrm{C}}$. The origin of this behavior is unclear; it may result from the compensation by interstitial $\mathrm{Mn}$ donors (Yamamoto and Katayama-Yoshida 1999, Mašek and Máka 2001) and/or from changes in the local spin configurations (van Schifgaarde and Mryasov 2001).

The temperature dependence of $M_{\mathrm{S}}$ determined by the magnetotransport measurements can be fitted rather well by the mean-field Brillouin function, as shown by the solid line in Fig. 13 (b) (Matsukura et al. 1998b). Owing to a moderate temperature and magnetic field dependence of $R_{\text {sheet, }}$ this conclusion remains valid assuming $R_{\text {Hall }} \approx c^{\prime} R_{\text {sheet }}^{2} M$. However, 
a rather different temperature dependence stems from the direct magnetization measurements (Fig. 11 (b)). To conjecture about the origin of this difference one should note that the anomalous Hall effect scales with the spin polarization of the carrier liquid. This polarization is proportional to the magnetization only if (i) the spin-splitting is much smaller than the Fermi energy and (ii) the contribution of the carriers to the total magnetization can be disregarded. Furthermore, the anomalous Hall effect does not provide information about the magnetization of the whole samples but only about its value in regions visited by the carriers. Just in these regions the carrier-mediated ferromagnetic interactions are strong. Thus, since near the metal-insulator boundary the carrier distribution is highly non-uniform, magnetotransport and direct magnetic measurements may provide different magnetization values (Dietl et al. 2000).

Parenthetically, no clear indication of the presence of MnAs clusters has been observed in the transport results, even in the cases where direct magnetization measurements detect their presence. One of possibilities is that the Schottky barrier formation around the MnAs clusters prevents their interaction with the carriers.

\subsubsection{Temperature and magnetic field dependence of resistivity}

The temperature dependencies of the resistivity $\rho$ in 200-nm thick layers of $\mathrm{Ga}_{1-x} \mathrm{Mn}_{x}$ As with different Mn content $x$ are displayed in Fig. 14. In terms of the metal-insulator transition (MIT), these films can be cost into two categories. Low- and high-Mn composition samples $(x<0.03, x>0.06)$ are on the insulator side of the MIT, whereas the layers containing intermediate $\mathrm{Mn}$ concentrations $(0.03 \leq x \leq 0.06)$ are metallic. All samples exhibit a negative magnetoresistan ce (MR) at low temperatures. Quite generally, the MIT occurs if the kinetic energy of holes at the Fermi level is high enough to prevent localization by disorder associated with the presence of $\mathrm{Mn}$ acceptors and compensating donors.

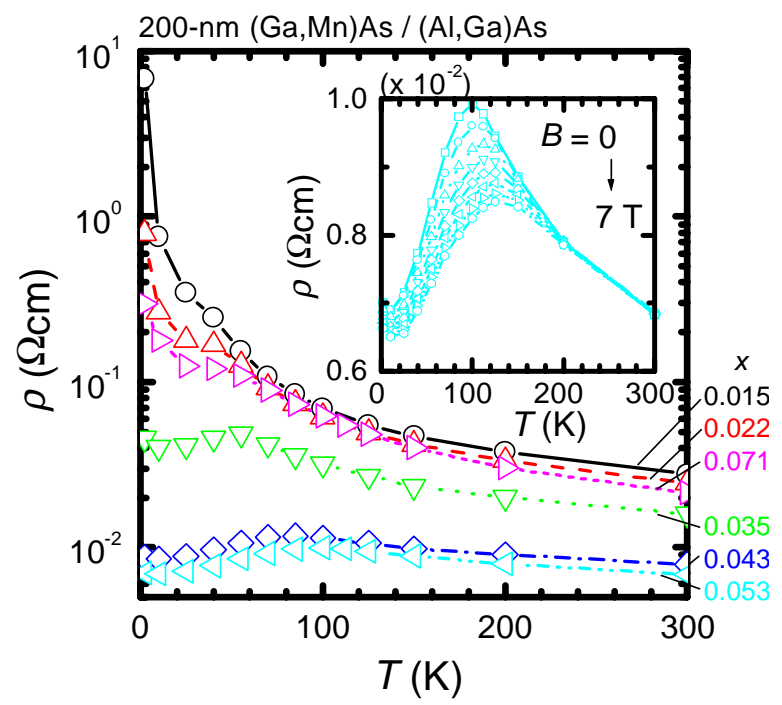

Fig. 14. Temperature dependence of the resistivity $\rho$ at zero magnetic field for $\mathrm{Ga}_{1-x} \mathrm{Mn}_{x}$ As films with $x=$ $0.015 \sim 0.071$. Samples with $x=0.035 \sim 0.053$ exhibit metallic behavior. The inset shows an expanded view for the sample with $x=0.053$ together with the dependence on the magnetic field (Matsukura et al. 1998b).
The temperature dependence of $\rho$ in the metallic (Ga,Mn)As samples show a maximum around $T_{\mathrm{C}}$ (the inset to Figs. 14), where the negative magnetoresistance also peaks; $\rho$ decreases by $20 \%$ in $B=7 \mathrm{~T}$ (at $100 \mathrm{~K}$ ) (see the insets to Figs. 13 (a) and 14). Such behavior suggests the presence of spin disorder scattering by thermodynamic fluctuations of the magnetization. In particular, the peak around $T_{\mathrm{C}}$ can be interpreted in terms of a critical scattering by packets of ferromagnetically coupled spins, whose correlation length is comparable to the wavelength of the carriers at the Fermi level (Matsukura et al. 1998b, Omiya et al. 2000). Such spin-disorder scattering may also perturb the position of the mobility edge, affecting the temperature dependence of the conductivity (Van Esch et al. 1997). The negative MR occurs because the field-induced spin-alignment reduces the spin-disorder scattering.

The effect of critical scattering on the resistance was evaluated assuming that the holes reside in a simple parabolic band (Omiya et al. 2000). The corresponding contribution to the resistivity has to take into account the presence of correlation between neighboring spins, $\left\langle\mathbf{S}_{i} \mathbf{S}_{j}\right\rangle \neq\left\langle\mathbf{S}_{i}^{2}\right\rangle \delta_{i j}$ (Dietl 1994),

$$
\rho_{\mathrm{S}}=2 \pi^{2} \frac{k_{\mathrm{F}}}{p e^{2}} \frac{m^{2} \beta^{2}}{h^{3}} \frac{k_{\mathrm{B}} T}{g^{2} \mu_{\mathrm{B}}^{2}}\left[2 \chi_{\perp}(T, B)+\chi_{/ /}(T, B)\right] .
$$

Here $k_{\mathrm{F}}$ is the Fermi wave vector determined from the value of the hole concentration $p$ assuming a spherical Fermi surface, $m$ is the hole effective mass taken as $0.5 m_{0}\left(m_{0}\right.$ is the free electron mass), $\beta$ is the exchange integral between the holes and the Mn spins, and $h$ is Planck's constant. The transverse and longitudinal magnetic susceptibilities are determined from the magnetotransport data according to $\chi_{\perp}=\partial M / \partial B$ and $\chi_{/ /}=M / B$.

As shown in Fig. 15, eq. (2) reproduces the high-temperature part of the temperature dependence of $\rho$ (Omiya et al. 2000); the data can be fitted by $\chi T$, the

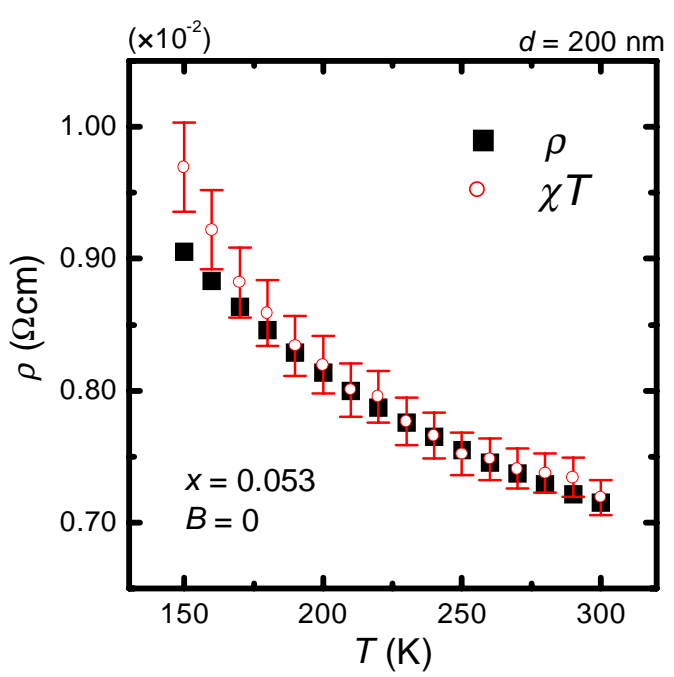

Fig. 15. Temperature dependence of the resistivity for a 200 -nm thick film of $\mathrm{Ga}_{1-x} \mathrm{Mn}_{x}$ As with $x=0.053$ in the high-temperature paramagnetic region. Solid squares and open circles show experimental data and the fitting using eq. (2), respectively (Omiya et al. 2000). 


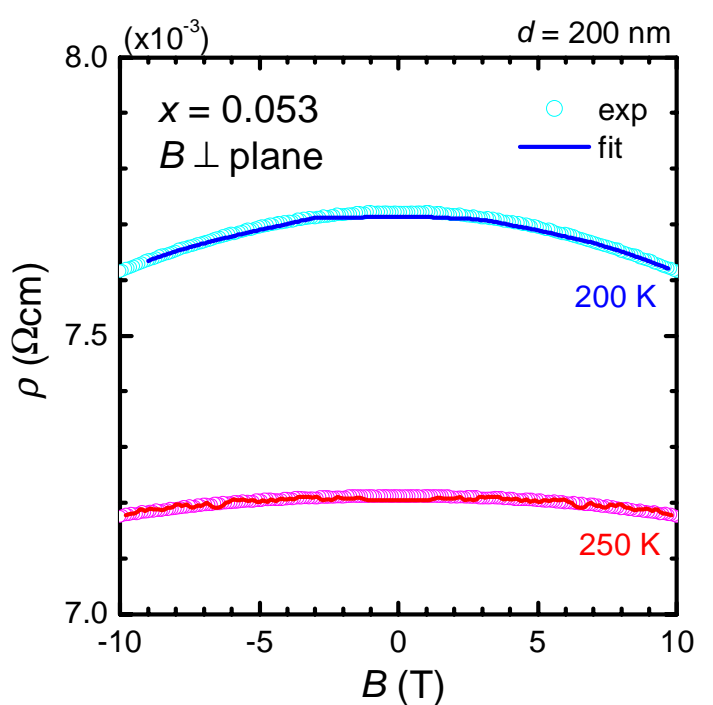

Fig. 16. Magnetic field dependence of the resistivity for a 200-nm thick film of $\mathrm{Ga}_{1-x} \mathrm{Mn}_{x}$ As with $x=0.053$ in the high-temperature paramagnetic region. The solid lines show the fitting using eq. (2) (Omiya et al. 2000).

temperature dependence characteristic for critical scattering. On approaching $T_{\mathrm{C}}$, however, the $\chi T$ fit deviates from the experimental points. This is, presumably, because no dependence of the magnetic susceptibility $\chi$ on the wave vector $q$ is taken into account. Since $\chi(q)$ is a decreasing function of $q$ in ferromagnetic materials, eq. (2) overestimates the critical scattering, particularly near $T_{\mathrm{C}}$, where $\chi(q=0)$ diverges. As shown in Fig. 16, the negative MR can be explained by a reduction of spin-dependent scattering in the magnetic field, according to eq. (2).

From the fit of eq. (2) to the temperature and field dependencies of $\rho$, the exchange energy $\left|N_{0} \beta\right|$ was evaluated by Omiya et al. (2000). Both $T$ and $B$ fits yield $\left|N_{0} \beta\right|$ of $1.5 \pm 0.2 \mathrm{eV}$. Though this value of the exchange energy compares favorably with that determined by photoemission experiments on (Ga,Mn)As, 1.0 1.2 eV (Okabayashi et al. 1998), it should be stressed that it has been obtained disregarding effects of localization. Another possible source of ambiguity is the complex valence band structure, not accounted for in eq (2).

A rather large negative MR, with a substantial anisotropy, has been observed in 'reentrant' insulating samples with high $x$ (Oiwa et al. 1998b, Katsumoto et al. 1998). A destruction of bound magnetic polarons, as invoked in an earlier work on (In,Mn)As (Ohno et al. 1992), and an increase in the hole kinetic energy due to giant spin-splitting, are suggested as mechanisms leading to the enhanced conductivity in the magnetic field. The latter can be particularly strong in p-type materials, in which the exchange splitting mixes heavy and light hole subbands (Wojtowicz et al. 1986).

The theoretical model describes transport properties of (Ga,Mn)As ( $T$ and $B$ dependence) by using Zubarev's double-time Green's function rather well (Kuivalainen 2001). The results show that the spin scattering (spin disorder scattering and critical scattering) as well as ionized impurity scattering have significant contributions to the total carrier mobility. For better understanding, an improvement of the theory is necessary, which includes the effect of disorder, the formation of polarons, etc.

Anisotropic magnetoresistance has also been

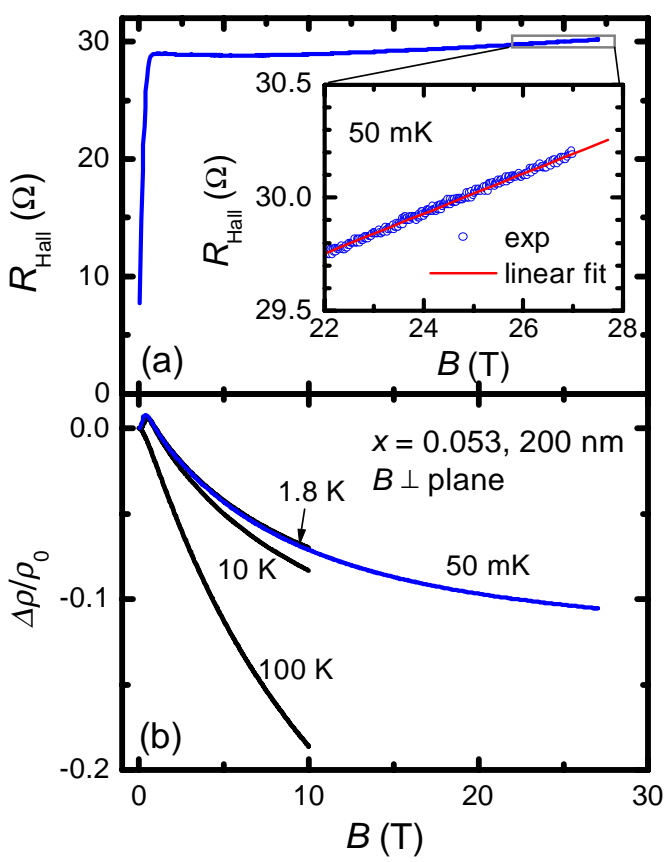

Fig. 17. Magnetotransport properties of a 200-nm thick film of $\mathrm{Ga}_{1-x} \mathrm{Mn}_{x}$ As with $x=0.053$ at $50 \mathrm{~m} \mathrm{~K}$ in high magnetic fields. (a) Hall resistance, which is a linear function of the magnetic field in the high-field region (inset). (b) Sheet resistance; negative magnetoresistance tends to saturate in the high-field region (Omiya et al. 2000).

observed in metallic ( $\mathrm{Ga}, \mathrm{Mn}) \mathrm{As}$, which depends on the relative direction between the magnetic field and the current. For (Ga,Mn)As with compressive strain grown on a GaAs buffer layer, the resistance is higher when the magnetic field is perpendicular to the measuring current than when the two are mutually parallel (Baxter et al. 2002). On the other hand, for $(\mathrm{Ga}, \mathrm{Mn}) \mathrm{As}$ with tensile strain grown on an (In,Ga)As buffer layer, the resistance is lower when a magnetic field perpendicular to the current (Sugawara et al. 1997). The origin of the anisotropic magnetoresistance is not clear yet.

Because of the dominance of the anomalous Hall term in wide temperature and field ranges, it is not straightforward to determine the carrier type and concentration in ferromagnetic semiconductors. Only at low temperatures and under very high fields, the anomalous Hall term saturates, so that the ordinary Hall coefficient can be determined from the remaining linear change of the Hall resistance in the magnetic field. Note that although $M$ saturates in relatively low magnetic fields, the negative MR usually persists, and generates the field dependence of the anomalous Hall coefficient.

Measurement of $R_{\text {Hall }}$ at $50 \mathrm{mK}$ in the field range of 22 27 $\mathrm{T}$ on the sample with $x=0.053$ revealed that the conduction is of $\mathrm{p}$-type, consistent with the acceptor character of $\mathrm{Mn}$. The determined hole concentration was $p=3.5 \times 10^{20} \mathrm{~cm}^{-3}$, about $30 \%$ of the nominal concentration of Mn (Fig. 17) (Omiya et al. 2000). A similar value of the hole concentration, which is almost independent of $x$, has been obtained from the Seebeck coefficient assuming a simple model of the valence band (Osinniy et al. 2001).

If all $\mathrm{Mn}$ centers are acting as acceptors in the metallic sample described above, $70 \%$ of them must have been compensated by donors. The most natural candidates for these donors are As antisite defects, which act as deep donors in GaAs. 
Accordingly, (Ga,Mn)As should become insulating at room temperature when the density of As antisites exceeds the density of shallow acceptors. Because the magnitudes of these densities are comparable and moreover fluctuate from run to run depending on subtleties of the growth conditions, we expect the overcompensation to occur occasionally. However, no such 'overcompensated' sample has been obtained so far. This seems to call for mechanisms controlling the upper limit of the excess As concentration (Luysberg et al. 1998) and/or leading to autocompensation of $\mathrm{Mn}$ but not to overcompensation (Walukiewicz 1988). One candidate for the latter might be the Mn interstitial, which is a donor according to first principles calculation (Yamamoto and Katayama-Yoshida 1999, Mašek and Máka 2001). Another candidate is the formation of disordered sixfold-coordinated centers with As, which would act as a donor (Van Esch et al. 1997).

Since the As antisite is anyway one of the most important defects acting as a compensating donor, the excess As influences substantially the magnetic and transport properties of (Ga,Mn)As. The increase of substrate temperature and the decrease of the As pressure reduces the density of excess As, which result in a decrease of the lattice constant and an increase in both the hole concentration and conductivity. Importantly, this generates a raise of $T_{\mathrm{C}}$ (Shimizu et al. 1999), confirming the critical role of band holes in the ferromagnetism of ( $\mathrm{Ga}, \mathrm{Mn}) \mathrm{As}$. The annealing of (Ga,Mn)As at relatively low temperatures ( $\leq$ $300 \mathrm{~K}$ ) leads to similar results due to the evaporation of excess As (Hayashi et al. 2001, Potashnik et al. 2001).

\section{2. (In,Mn)As}

\subsection{1. n-type (In,Mn)As}

Magnetization measurements reveal that n-type (In,Mn)As layers with $x=0.046 \sim 0.18$ grown at $200^{\circ} \mathrm{C}$ are paramagnetic. The analysis in terms of the Curie-Weiss law shows that the interaction between the $\mathrm{Mn}$ ions is antiferromagnetic with the nearest-neighbor exchange $J_{\mathrm{nn}}=-1.9$ K (von Molnár et al. 1991). The effective magnetic moment of Mn is determined as $p_{\text {eff }}(=g \sqrt{S(S+1)})=5.49$ for $x=0.046$, indicating that the Mn electrons are mostly in the high spin $\mathrm{d}^{5}$ configuration.

Magnetotransport measurements on n-type samples show no evidence for carrier-Mn spin interactions (Ohno et al. 1991). In particular, no magnetization-dependent anomalous Hall effect is observed within the experimental error.

\subsection{2. p-type (In,Mn)As}

p-type (In,Mn)As shows ferromagnetism at low temperatures. Magnetotransport measurements of thick p-type (In,Mn)As films grown on (001) semi-insulating GaAs reveals that the temperature dependence of the Hall coefficient, $R_{\mathrm{H}}$, can be expressed, as is the case of (Ga,Mn)As, by eq. (1) (Ohno et al. 1992). Since $M=\chi H, B=\mu_{0} H, \chi=C /(T-\theta)$ in the paramagnetic temperature region, and assuming $R_{\mathrm{S}}=c \rho$ one obtains the Hall coefficient as,

$$
R_{\mathrm{H}}\left(=\rho_{\text {Hall }} / B\right)=R_{0}+c \rho \chi / \mu_{0},
$$

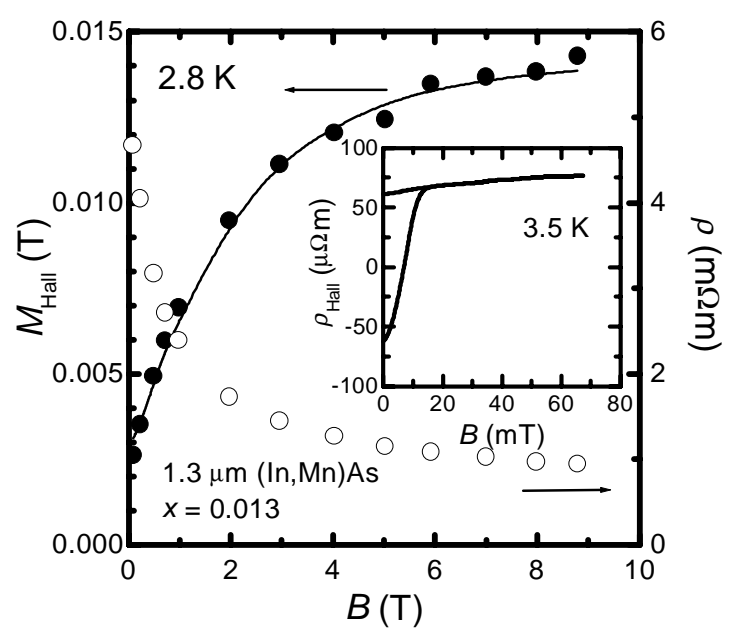

Fig. 19. Magnetic field dependence of the diagonal resistivity $\rho$ (open circles) and magnetization $M_{\text {Hall }}$ (close circles) determined from the ratio of the Hall and diagonal resistivities, $M_{\text {Hall }}=\rho_{\text {Hall }} / c \rho$, where $c=6.3$, for a $1.3-\mu \mathrm{m}$ thick film of $\operatorname{In}_{1-x} \mathrm{Mn}_{x}$ As with $x=0.013$. The solid line is a fit by the modified Brillouin function $B_{\mathrm{S}}(y)$, where $S=5 / 2$ and $y=$ $S g \mu_{\mathrm{B}} B /\left(T+T_{\mathrm{o}}\right)$ with $T_{\mathrm{o}}=1.5 \mathrm{~K}$. The inset shows the hysteresis observed in the Hall resistivity at 3.5 K (Ohno et al. 1992).

where the Hall resistivity $\rho_{\text {Hall }}=d R_{\text {Hall }}$. Equation (3) can describe the temperature dependence of $R_{\mathrm{H}}$ of a $1.3-\mu \mathrm{m}$ thick (In,Mn)As sample $(x=0.013)$ grown directly on a GaAs substrate at $275^{\circ} \mathrm{C}$, especially from 20 to $200 \mathrm{~K}$, as shown in Fig. 18. The fit carried out by adopting the calculated value of the Curie constant (using $x=0.013$ and assuming Mn spin $S=5 / 2$ ) yields $R_{0}, c$ and $\theta$, which are $p=2.2 \times 10^{19} \mathrm{~cm}^{-3}$ (determined for $\left.\mathrm{m} R_{0}\right), c=5.6$, and $\theta=3.8 \mathrm{~K}$. The sign of $\theta$ demonstrates that the interaction between the $\mathrm{Mn}$ spins is ferromagnetic. A ferromagnetic Mn-Mn interaction has only been observed in p-type samples so far, proving that this interaction is induced by the presence of the holes. Equation (1) is shown to be valid at low temperatures also, where hysteresis appear in the field dependence of $\rho_{\text {Hall, }}$, as shown in the inset to Fig. 19. This points to the presence of ferromagnetic order. By comparing the

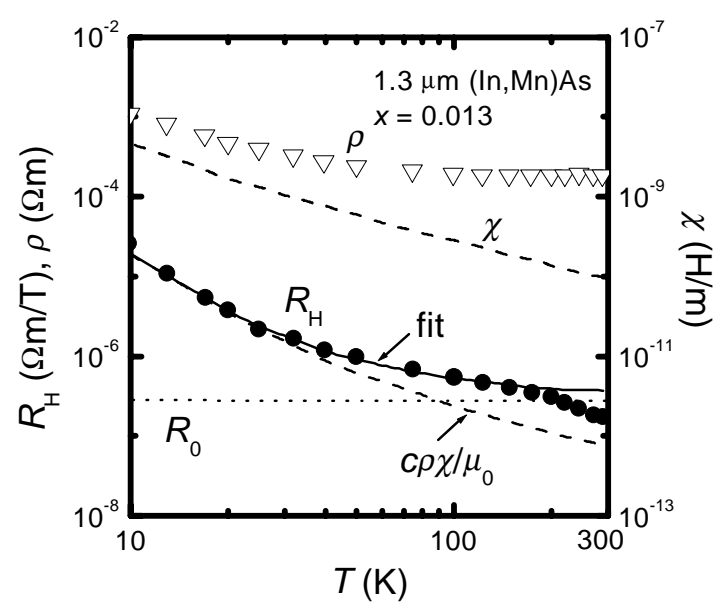

Fig. 18. Temperature dependence of the Hall coefficient $R_{\mathrm{H}}$ and resistivity $\rho$ of a $1.3-\mu \mathrm{m}$ thick film of $\operatorname{In}_{1-x} \mathrm{Mn}_{\mathrm{x}} \mathrm{As}$ with $x=$ 0.013. $R_{\mathrm{H}}$ can be modeled over a wide range of temperatures as $R_{0}+c \rho \chi / \mu_{0}$, which is shown by the solid line assuming $c=5.6$. The susceptibility $\chi$ (depicted by the dashed line) is calculated assuming the Curie-Weiss law with $x=0.013$, Mn spin $S=5 / 2$, and the Curie-Weiss temperature $\theta=3.8 \mathrm{~K}$ (Ohno et al. 1992). 
remanent magnetization determined from SQUID measurements at low temperatures with the temperature dependence of the remanent part of $\rho_{\text {Hall }} \rho\left(\rho_{\text {Hall }} \approx R_{\mathrm{S}} M=c \rho M\right)$ as shown in Fig. 20, one can again evaluate the proportionality constant $c$ as 6.3 , in good agreement with $c=5.6$ obtained from the $\mathrm{p}$ aramagnetic temperature region. This indicates that the mechanism responsible for the anomalous Hall effect does not change between the two temperature ranges.

The magnetic field dependence of $M_{\text {Hall }}\left(=\rho_{\text {Hall }} / R_{\mathrm{S}}\right)$ (i. e., magnetization measured by the anomalous Hall effect) can be expressed as a sum of ferromagnetic and paramagnetic terms. The latter can be described by a modified Brillouin function (Gaj et al. 1979), as shown in Fig. 19. The magnitude of saturation magnetization (the sum of the two responses at $B=$ $0.015 \mathrm{~T}$ at $T=3.5 \mathrm{~K}$ ) is $0.014 \mathrm{~T}$, just the value expected for $x=$ 0.013 and $S=5 / 2$.

\subsubsection{Pseudomorphic p-type (In,Mn)As}

The (In,Mn)As layers grown on quasi-lattice matched buffer layer ( $\mathrm{GaSb}$ or $(\mathrm{Al}, \mathrm{Ga}) \mathrm{Sb})$ films are either superparamagnetic or ferromagnetic at low temperatures depending on the initial growth mode (Shen et al. 1997c). They have rather high $T_{\mathrm{C}}(=50 \mathrm{~K})$ with $x \sim 0.07$ and relatively low $p$ $\left(\sim 2-3 \times 10^{19} \mathrm{~cm}^{-3}\right.$, determined from Hall coefficient at room temperature) (Slupinski et al. 2002b). The magnetotransport properties show no clear evidence for partial ferromagnetism, that is for the co-existence of ferromagnetism and paramagnetism in the same films. A comparison of transport and magnetization measurements revealed that the anomalous Hall coefficient $R_{\mathrm{S}}$ is rather proportional to $\rho^{2}$ than to $\rho$ in the low-temperature regime (Oiwa et al. 1999). Surprisingly, in contrast to the case of (Ga,Mn)As and (In,Mn)As with high hole concentrations, the sign of the anomalous Hall effect was found to be negative in (In,Mn)As films with low hole concentrations (Munekata et al. 1997). Such a sign reversal appears to be possible if skew-scattering by compensating impurities dominates (Leroux-Hugon and Ghazali 1972, Chazalviel 1975, Dorleijn 1976).

The magnetic anisotropy in (In,Mn)As has been investigated for various strains in the film, by means of varying the lattice constant of the buffer layer (Munekata et al. 1993). To increase the tensile strain, $(\mathrm{Al}, \mathrm{Ga}) \mathrm{Sb}$ with high $\mathrm{Al}$ composition was employed, whereas to decrease its magnitude, $\mathrm{Al}(\mathrm{Sb}, \mathrm{As})$ was used as a buffer layer. The corresponding reduction of the tensile strain diminishes the perpendicular magnetic anisotropy, which demonstrates that strain is indeed responsible for the magnetic anisotropy. At the same time, no changes in $T_{\mathrm{C}}$ are observed upon changing strain (Fumagalli and Munekata 1996). The thickness of the (In,Mn)As film affects also the magnitude of the magnetic anisotropy. For thick samples there is no magnetic anisotropy due to the relaxation of the lattice mismatch, whereas for very thin samples there is no ferromagnetic order due to the depletion of the holes. The thick (In,Mn)As films without anisotropy exhibit long-term magnetization relaxation at low temperatures, which suggests that a cluster-glass state is formed (Oiwa et al. 1999).

The magnetic properties of structures consisting of an (In,Mn)As layer grown onto an (In,Al)As with an composition of $\mathrm{Al} \sim 0.15$ and an AlSb buffer layer were examined by means of the anomalous Hall effect. A weakening of hysteresis with the increase of the intermediary (In,Al)As layer thickness was observed. This may result from a decrease of overlap between the Mn spins in the (In,Mn)As layer and the holes accumulated at the (In, Al)As/AlSb heterointerface (Munekata et al. 1992).

\subsection{Infrared and far infrared optical conductivity}

Infrared (IR) and far infrared (FIR) transmission spectra were collected between 10 and $12000 \mathrm{~cm}^{-1}$ for two (Ga,Mn)As samples with metallic dc conductivity (Nagai et al. 2001). The thickness and Mn concentration of these two films were $2 \mu \mathrm{m}, x=0.034$ and $0.4 \mu \mathrm{m}, x=0.050$, respectively. In the whole spectral region studied, the absorption coefficient is larger for the sample with the higher Mn composition. In the absorption spectrum of the sample with $x=0.034$, a broad peak is observed around $1600 \mathrm{~cm}^{-1}(200 \mathrm{meV})$. Most probably, this peak reflects both photo-ionization transitions between $\mathrm{Mn}$ impurity states and the valence band as well as inter-valence band transitions, which have been also observed in the IR absorption spectrum of bulk GaAs:Mn with a Mn concentration of $4 \times 10^{17} \mathrm{~cm}^{-3}$ (Chapman and Hutchinson 1967). There exists also quite a different interpretation, which assigns the peak to the formation of polarons (Katsumoto et al. 2001). This peak is less visible in the sample with $x=0.050$, presumably because of a greater degree of overlap among the Mn impurity states, which leads to hole delocalization. In the FIR region, the absorption increases monotonously with the photon energy, and no clear Drude conductivity is observed down to $10 \mathrm{~cm}^{-1}$. This behavior may reflect the influence of quantum localization effects on the Drude conductivity as well as the coexistence of the hopping and Drude terms in the vicinity of the MIT (Liu et al. 1993, Gaymann et al. 1995).

The dependence of the absorption coefficient on temperature and the magnetic field in the FIR region is consistent with the corresponding behavior of the d.c. conductivity. In particular, the optical density shows both a minimum around $T_{\mathrm{C}}$ and an increase in the magnetic field. When the absorption spectrum at $T_{\mathrm{C}}$ is subtracted from the spectra below $T_{\mathrm{C}}$, a Drude-like absorption is obtained below 400 $\mathrm{meV}$, whose magnitude increases as temperature decreases. The Drude-like absorption and localization-induced effects are observed also in (In,Mn)As (Hirakawa et al. 2001). A detected shift of the spectral density towards higher energies was taken as an evidence for the double exchange spin-spin interaction mechanisms (Hirakawa et al. 2001). This conclusion awaits a verification by considering the behavior of the sum rule in the case of the narrow-gap and complex valence band structure, specific to p-type (In,Mn)As.

Theoretical simulations for the optical spectra were also been performed (Sakai et al. 2001, Sakai and Suzuki 2001).

\subsection{Cyclotron resonance}

Magneto-transmission spectra of GaAs:Mn with $\mathrm{Mn}$ a concentration of $\sim 9 \times 10^{17} \mathrm{~cm}^{-3}$ grown by MBE were taken at several temperatures in magnetic fields up to $110 \mathrm{~T}$ by the use of a FIR laser line of $119 \mu \mathrm{m}(10.4 \mathrm{meV})$ and $70.5 \mu \mathrm{m}(17.6$ meV) (Matsuda et al. 1998). It was found that the carrier 
mobility is too low above $270 \mathrm{~K}$ to observe the cyclotron resonance $(\mathrm{CR})$ and that carrier freeze-out occurs below $140 \mathrm{~K}$. In the intermediate temperature region a $\mathrm{CR}$ peak was observed in $\sim 40 \mathrm{~T}$ at $119 \mu \mathrm{m}$. The mobility determined from the $\mathrm{CR}$ linewidth at $220 \mathrm{~K}$ is $780 \mathrm{~cm}^{2} / \mathrm{Vs}$. The cyclotron mass is $\sim 0.42$ $m_{0}$ and $\sim 0.47 m_{0}$ at $119 \mu \mathrm{m}$ and $70.5 \mu \mathrm{m}$, respectively.

On the other hand, 150-nm thick (Ga,Mn)As with $x$ $=0.053\left(T_{\mathrm{C}}=88 \mathrm{~K}\right)$ shows no $\mathrm{CR}$ up to $150 \mathrm{~T}$ at $119 \mu \mathrm{m}$, which may result from too low mobility value. In the investigated temperature range from 20 to $150 \mathrm{~K}$, the transmission decreases with the magnetic field, this behavior being consistent with the negative magnetoresistance observed in such samples. The spin origin of the effect is corroborated by the fact that the magnetic field at which the transmission ceases to change corresponds with to the saturation field of the magnetization (Matsuda et al. 1998).

The CR of n-type (In,Mn)As layers with $x=0,0.08$, and 0.12 was observed at $5.5 \mu \mathrm{m}(224 \mathrm{meV})$ and $10.6 \mu \mathrm{m}(117$ $\mathrm{meV})$. The cyclotron mass at $224 \mathrm{meV}$ decreases from $0.054 m_{0}$ to $0.048 m_{0}$ for $x$ increasing from 0 to 0.12 . The conduction-band effective mass depends also on temperature. For example, for the $x=0.08$ sample, it decreases from $0.051 m_{0}$ at room temperature to $0.045 m_{0}$ at $18 \mathrm{~K}$. For $1-\mu \mathrm{m}$ thick (In,Mn)As with $p \sim 10^{19} \mathrm{~cm}^{-3}$, the $\mathrm{CR}$ of the light-hole was detected (Matsuda et al. 2001).

\section{Magneto-optical properties}

Magneto-optical properties of (In,Mn)As and (Ga,Mn)As have been studied in order to elucidate the origin of ferromagnetism as well as to explore the possibility of using these materials as Faraday isolators, suitable for monolithic integration with the existing semiconductor lasers.

The absorption edge of (Ga,Mn)As is not sharp, as shown in Fig. 20 (Kuroiwa et al. 1998, Szczytko et al. 1999b). This is probably due to impurity band formation caused by the high concentration of ionized $\mathrm{Mn}$ and compensating donors (Kuroiwa et al. 1998). Even below the fundamental absorption edge, the absorption coefficient is rather large due to free-carrier

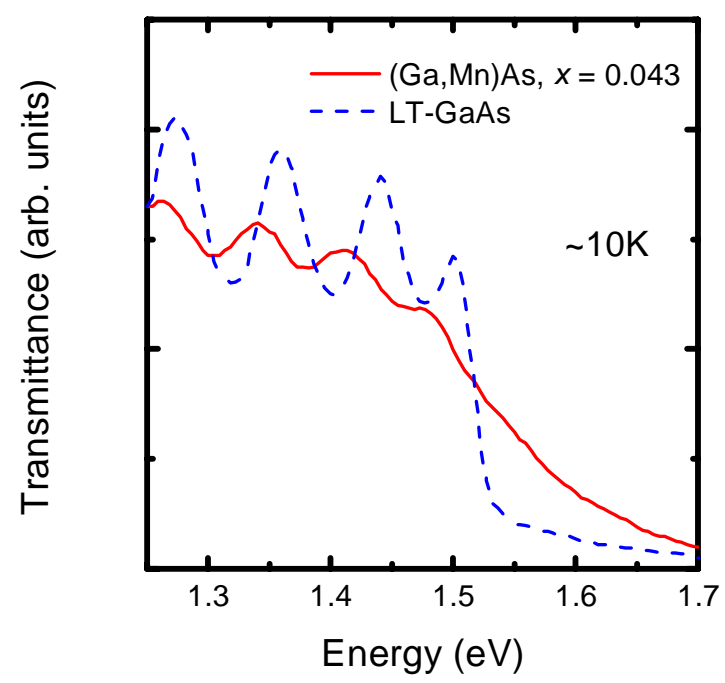

Fig. 20. Optical transmission spectra of $2-\mu \mathrm{m}$ thick films of $\mathrm{Ga}_{1-x} \mathrm{Mn}_{x}$ As with $x=0.043$ and low-temperature grown GaAs at $\sim 10$ K. (Kuroiwa et al. 1998).
(Casey et al. 1975) and intra-Mn absorption. There is no report on the observation of exciton states or photoluminescence, which is probably due to non-radiative recombination, carrier screening, and the formation of an impurity band (Ando et al. 1999).

\subsection{Faraday rotation}

Figure 21 (a) shows Faraday rotation spectra of (Ga,Mn)As $(2 \mu \mathrm{m}, x=0.043)$ at 10 and $300 \mathrm{~K}$ in the vicinity of the band gap energy (Kuroiwa et al. 1998). Compared to GaAs, the magnitude of the Faraday rotation in $(\mathrm{Ga}, \mathrm{Mn}) \mathrm{As}$ is very large and proportional to the magnetization, as shown in Fig. 21 (b). The observed value of the Faraday rotation is $6 \times 10^{4} \% \mathrm{~cm}$ in $0.1 \mathrm{~T}$ at $10 \mathrm{~K}$, and the room temperature Verdet constant is $9 \times 10^{-2} \% / \mathrm{G} \mathrm{cm}$. Oscillations seen in the low-energy region at 10 $\mathrm{K}$ are caused by interference originating from internal multiple reflections.

The Faraday rotation angle $\theta_{\mathrm{F}}$ can be expressed as,

$$
\theta_{\mathrm{F}} \approx \frac{E d}{2 \hbar c} \frac{\partial n}{\partial E} \Delta E,
$$

where $E$ is the photon energy, $c$ is the light velocity, $n$ is the refractive index, and $\Delta E$ is the energy difference of the transitions for the two circular light polarizations. The value of $\Delta E$ inferred from eq. (4), using experimentally determined
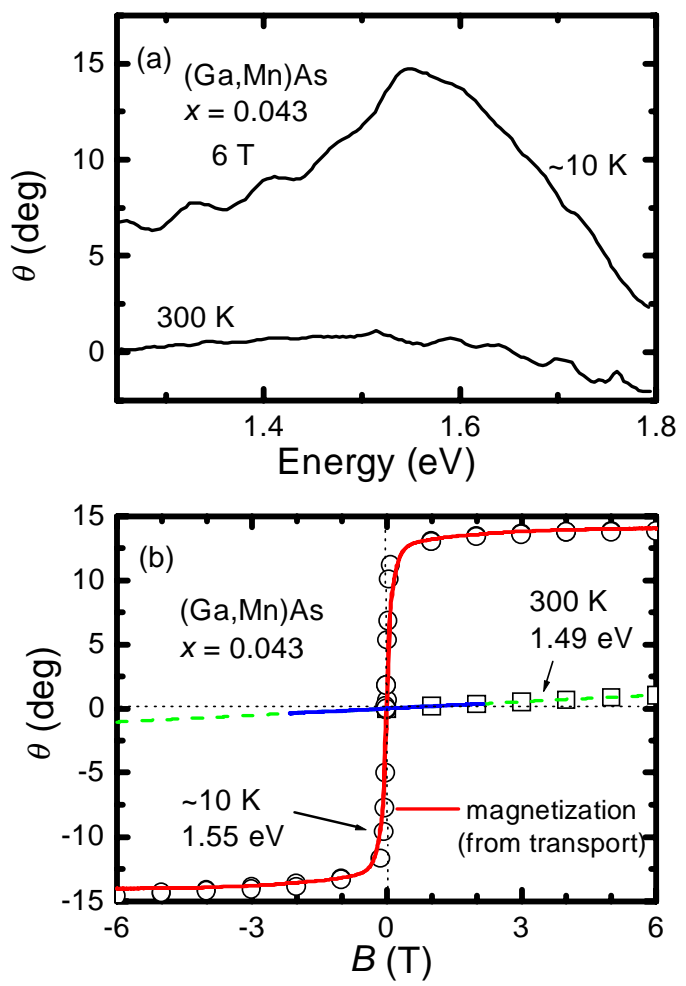

Fig. 21. Faraday rotation for a $2-\mu \mathrm{m}$ thick film of $\mathrm{Ga}_{1-x} \mathrm{Mn}_{x}$ As with $x=0.043$ measured as a function of the photon energy in a magnetic field of $6 \mathrm{~T}$ at $\sim 10$ and $300 \mathrm{~K}$ (a) and as a function of the magnetic field at $\sim 10 \mathrm{~K}, 1.55 \mathrm{eV}$ and at $300 \mathrm{~K}, 1.49 \mathrm{eV}$ (b). Solid lines show the magnetization determined from magnetotransport measurements at the given temperatures (scaled to match the open symbols) (Kuroiwa et al. 1998). 
$\partial n / \partial E$, is about $35 \mathrm{meV}$, fairly independent of $E$ between 1.25 and $1.6 \mathrm{eV}$. If one uses $\Delta E=\left(N_{0} \beta-N_{0} \alpha\right) x\left\langle S_{z}\right\rangle$, which is valid only at the band edge, then $\left(N_{0} \beta-N_{0} \alpha\right) \approx 1 \mathrm{eV}$ is obtained. Here $N_{0} \alpha$ is the exchange integral for the conduction band, and $\left\langle S_{z}\right\rangle$ the thermal average of the Mn spins in the direction of $B$, determined by an independent magnetization measurement. The positive value of $\left(N_{0} \beta-N_{0} \alpha\right)$ reflects the positive sense of the Faraday rotation, which is opposite to that of II-VI DMSs like undoped $(\mathrm{Cd}, \mathrm{Mn}) \mathrm{Te}$. This surprising result is explained if a large Burstein-Moss shift due to the high hole concentration specific to III-V DMS is taken into consideration (Szczytko et al. 1999b, Dietl et al. 2001c).

The magnetic field dependence of the Kerr-rotation in (Ga,Mn)As at low-temperature shows clear hysteresis reflecting the ferromagnetic order (Sadowski et al. 2001d). A study on reflectivity and polar Karr-rotation of thin (In,Mn)As films grown on $(\mathrm{Al}, \mathrm{Ga}) \mathrm{Sb}$ buffer layers showed that the squareness (the ratio of the Kerr-rotation angle at remanence to that at saturation) can be as high as $100 \%$ (Fumagalli and Munekata 1996).

\subsection{Magnetic circular dichroism (MCD)}

Magnetic circular dichroism (MCD) is caused by the difference in the magnitude of photon absorption for left-polarized $\left(\sigma^{-}\right)$and right-polarized $\left(\sigma^{+}\right)$light,

$$
\mathrm{MCD}=\left(\alpha^{-}-\alpha^{+}\right) /\left(\alpha^{-}+\alpha^{+}\right) \approx\left(T^{+}-T\right) /\left(T^{+}+T\right),
$$

where $\alpha^{+}\left(T^{+}\right)$and $\alpha^{-}(T)$ are the absorption (transmission) coefficients for right and left circularly polarized light, respectively. The incorporation of Mn enhances the MCD signal at optical critical points as shown in Fig. 22 (Ando et al. 1998). According to MCD studies of $(\mathrm{Ga}, \mathrm{Mn}) \mathrm{As}$ in the reflectivity mode near the $E_{0}$ critical point, the splitting of the valence band points to a negative $N_{0} \beta$ value, similarly to the case of II-VI DMS. On the other hand, a positive value of MCD deduced from absorption measurements appears to suggest that $N_{0} \beta$ is positive (Szczytko et al. 1996, Shimizu et al. 1998, Beschoten et al. 1999). As already mentioned, a larger absorption coefficient for the left light polarization can be reconciled with the negative value of $N_{0} \beta$ by taking into account the Moss-Burstein shift (Szczytko et al. 1999b, Dietl et al. 2001c). This mechanism operates also on the insulator side of the MIT, that is in the case of samples with small Mn concentrations, provided that the intra-center correlation energy $U$ of $\mathrm{Mn}$ acceptors is smaller than the Fermi energy in the impurity band.

In addition to the positive MCD, a negative contribution of a magnitude that increases with decreasing temperature, was observed in ferromagnetic (Ga,Mn)As (Beschoten et al. 1999). This peculiar temperature dependence can be explained in the framework of the standard model of interband transitions in doped DMS. It results from a non-linear dependence of MCD on magnetization at the Moss-Burstein edge (Dietl et al. 2001c). In order to describe the experimental data more quantitatively, a model taking into account the effect of disorder on the transition probability was also proposed (Szczytko et al. 2001b).

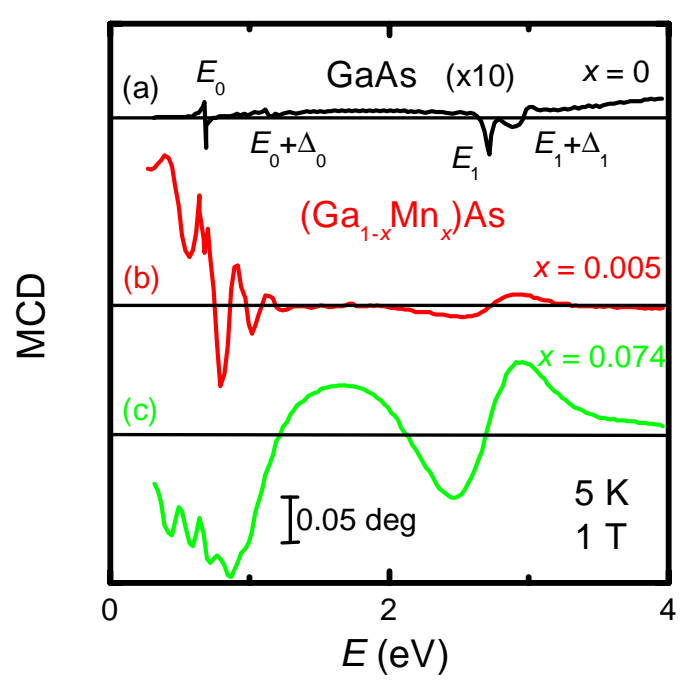

Fig. 22. Magnetic circular dichroism (MCD) spectra of (a) undoped semi-insulating GaAs substrate and (b), (c) of epitaxial $\mathrm{Ga}_{1-x} \mathrm{Mn}_{x}$ As films at $T=55 \mathrm{~K}$ and $B=1 \mathrm{~T}$. The spectrum of GaAs is magnified ten times because the signal is weaker than that of $\mathrm{Ga}_{1-x} \mathrm{Mn}_{x} \mathrm{As}$ (Ando et al. 1998).

\section{Origin of ferromagnetism}

Despite a considerable effort aiming at elucidating the nature of ferromagnetism in III-V magnetic semiconductors, the form of the relevant minimal Hamiltonian and its universality for all III-V compounds are still under an active debate. Such a situation reflects the multifaceted environment, in which the ferromagnetism appears. Indeed, conceptual and technical difficulties inherent to theory of strongly correlated and disordered transition metal compounds are combined-in III-V magnetic semiconductors-with the intricate physics of Anderson-Mott localization that is specific to heavily doped semiconductors. Moreover, low-temperature epitaxy, by which most of the materials is obtained, results in a large concentration of native defects such as antisites, which act as compensating donors. Another possible source of local bond reconstructions is the mechanism of self-compensation, occurring in heavily doped semiconductors once the Fermi level reaches the energy triggering defect reactions. Structural faults may form with neighbor transition metal impurities defect complexes exhibiting hitherto unexplored magnetic characteristics. At the same time, strong compensation by donor-like defects enhances the electrostatic disorder substantially, leading to deep and long-range potential fluctuations that result in significant band tailing.

In this section, we first discuss predictions of $a b$ initio computations in comparison to photoemission and $\mathrm{x}$-ray magnetic-circular dichroism data, discussed already in the previous sections. Theoretical models employing parameterized Hamiltonians are presented next emphasizing their successes and shortcomings in the description of magnetic phenomena.

\subsection{First-principles studies}

$A b$ initio computations of the band structure in zinc-blende ferromagnetic semiconductors were initiated by Shirai et al. (1998), who employed the full potential augmented plane wave (FLAPW) method within the local-spin density approximation (LSDA) for MnAs and for a supercell structure of $\mathrm{Ga}_{1-x} \mathrm{Mn}_{x} \mathrm{As}$ 
with $x=1 / 2$. The total energy calculation demonstrated that the ferromagnetic ground state has indeed lower energy comparing to competing magnetic states and led to the correct value of the lattice constant. This points to the usefulness of LDSA for the determination of the ground state properties of the compounds in question. Later, the same group (Ogawa et al. 1999) adopted the linear muffin-tin orbital (LMTO) atomic sphere approximation (ASA) and essentially reproduced the results for $x=1$ as well as extended the previous computations to ordered alloys with $x=1 / 8$ and $1 / 4$. It has been noted that the band structure is half-metallic for the ferromagnetic state, i.e., the Fermi level lies in-between the densities of states corresponding to the majority spin-down and minority spin-up states. Furthermore, owing to the p-d hybridization there appears a magnetic moment on the As sites. The As and Mn moments are antiparallel, which confirms that the coupling between the carriers residing on the anion orbitals and the Mn spins is antiferromagnetic, $N_{0} \beta<0$. At the same time, the magnetic moment within the Mn muffin-tin radius is reduced to about $4 \mu_{\mathrm{B}}$ This may suggest that the $\mathrm{Mn}$ ion is in $\mathrm{d}^{4}$ state, i.e. that the holes have largely d-like character. This is not the case, however, according to the population analysis carried out by Sanvito et al. (2001), who employed the linear combination of atomic orbitals (LCAO) within LSDA for ab intio studies of $\mathrm{Ga}_{1-x} \mathrm{Mn}_{x} \mathrm{As}$. The results of Sanvito et al. (2001) imply that the charge accumulated on the $\mathrm{Mn}$ d-shell is about 5.5 in the electron charge units. This demonstrates that a transfer of electrons from the $3 \mathrm{~d} \mathrm{Mn}$ shell to sp bonds is actually outweighed by a transfer in the opposite sense, resulting in a partial occupation of the $3 \mathrm{~d}^{6}$-like state and a corresponding reduction of the Mn magnetic moment.

The supercell sizes adopted by Sanvito et al. (2001) corresponded to a wide range of $\mathrm{Mn}$ concentrations in $\mathrm{Ga}_{1-x} \mathrm{Mn}_{x} \mathrm{As}$, for which $N_{\mathrm{o}} \beta$ was evaluated. A surprising result is that the apparent value of the $\mathrm{p}-\mathrm{d}$ exchange energy $N_{0} \beta^{*}$, determined from the computed spin splitting of the valence band at the $\Gamma$ point of the Brillouin zone, increases from $-5.5 \mathrm{eV}$ for $x$ $=0.06$ to $-8.2 \mathrm{eV}$ for $x=0.02$. This dependence Sanvito et al. (2001) interpret in terms of the model put forward by Benoit à la Guillaume et al. (1992), who considered the case of strong and attractive "chemical" and exchange potentials introduced by the $\mathrm{Mn}$ atoms in II-VI DMS. It has been found that since in such a case the hole wave function tends to concentrate around the Mn ions, the virtual-crystal and molecular-field approximations cease to be valid. As a result, a bowing of the energy gap and an enhancement of the spin splitting is expected, particularly in the range of small Mn concentrations $x$. In an extreme case, the Mn can localize a hole, like $\mathrm{Cu}$ ions in under-doped cuprate superconductors (Zhang and Rice 1988). By fitting the computed valence-band splitting to the model of Benoit à la Guillaume et al. (1992), Sanvito et al. (2001), evaluated the bare value of the exchange energy $N_{0} \beta=-4.5 \pm 0.3 \mathrm{eV}$ and the "chemical" (spin-independent) part of the total Mn potential to be close to zero. For these parameters, the holes are not yet localized in the Zhang-Rice states but certainly the local potential will significantly contribute to the binding energy of the Mn acceptor state. As discussed in Sec. 4, the experimental values of $\left|N_{0} \beta\right|$ are smaller, $N_{0} \beta \approx-1 \mathrm{eV}$.

More recently, Zhao et al. (2001a) employed the FLAPW method within the generalized gradient approximation (GGA) to investigate further electronic and magnetic properties of ordered $\mathrm{Ga}_{1-x} \mathrm{Mn}_{x}$ As alloys with low and high Mn concentrations, 0.031 $\leq x \leq 0.5$. The determined Mn moment in the muffin tin (MT) sphere at given $x$ was found to be in a good agreement with the results of Ogawa et al. (1998). This indicates that LSDA and GGA agree with each other very well in the description of magnetic properties for a given $\mathrm{Ga}_{1-x} \mathrm{Mn}_{x}$ As structure. At the same time, the occupancy analysis indicates that the charge accumulated on the d-shell in the MT sphere is 5.2, again greater than 5 , although slightly smaller than that obtained by Sanvito et al. (2001), as quoted above. Furthermore, the difference in total energies of antiferromagnetic and ferromagnetic ground states, normalized to one $\mathrm{Mn}$ atom, was found to increase from $0.12 \mathrm{eV}$ for $x=0.0625$ to $0.24 \mathrm{eV}$ for $x=$ 0.5. This confirms that enlargement of Mn content in III-V compounds might constitute a road towards higher Curie temperatures $T_{\mathrm{C}}$.

The question about the performance of other transition metals in synthesizing functional ferromagnetic III-V semiconductors was addressed by Shirai (2001), who extended FLAPW computation within LSDA to zinc blende arsenides, MAs, where $\mathrm{M}=\mathrm{Ti}, \mathrm{V}, \mathrm{Cr}, \mathrm{Mn}, \mathrm{Fe}, \mathrm{Co}$ and $\mathrm{Ni}$. It was found that the ferromagnetic order is more stable than the antiferromagnetic state for VAs, CrAs, and MnAs, the greatest energy gain being predicted for CrAs. Another interesting question concerns ground state properties of hosts other than GaAs. Kato and Katayama-Yosida (1999) carried out first principles studies of ordered $\mathrm{Ga}_{0.75} \mathrm{Mn}_{0.25} \mathrm{~N}$, $\mathrm{Ga}_{0.75} \mathrm{Mn}_{0.25} \mathrm{~N}_{0.75} \mathrm{O}_{0.25}$, and $\mathrm{Ga}_{0.75} \mathrm{Fe}_{0.25} \mathrm{~N}$ alloys within the LAPW-LSDA scheme. The hole-mediated ferromagnetic ordering is favored in $(\mathrm{Ga}, \mathrm{Mn}) \mathrm{N}$, as confirmed in the later work by Sato and Katayama-Yosida (2001c), but for the remaining two alloys, in which there are no carriers, an aniferromagnetic ground state is predicted. Ordered alloys of $\mathrm{Ga}_{1-x} \mathrm{Mn}_{x} \mathrm{~N}$ with several Mn concentrations were studied by Kulatov et al. (2001) employing the tight binding LMTO-ASA-LSDA approach. The Mn 3d band was found to reside inside the band gap and to hybridize rather weakly with the band states. Nevertheless, the ground state appears to be ferromagnetic, and its stability -in contradiction to results of Kato and Katayama-Yoshida (1999) mentioned above-increases with n-type doping but decreases with p-type doping.

The first principles studies referred to above have stimulated interesting discussions about the accuracy of the adopted approximations. In particular, a question arises to what extend supercell models as well as the LSD and scalar-relativistic approximations are valid in the case of magnetic alloys in question. Akai (1998), by using the Korringa-Kohn-Rostoker (KKR) within LSDA approximation and describing chemical disorder in the cation sublattice by the coherent-potential approximation (CPA), obtained information about the ground state energy of the random $\mathrm{In}_{1-x} \mathrm{Mn}_{x}$ As alloy with $x=0.06$. Again, the ferromagnetic phase was found to have lower energy than the local-moment disordered state. Similarly to the results of the supercell LSDA calculations discussed above, the ferromagnetic state is half metallic, and the states near the Fermi energy contain a d-like component. On this ground, Akai (1998) assigns the ferromagnetism to the double exchange mechanism (Zener 1951b). Another important aspect of Akai's work is the direct demonstration that ferromagnetism 
is unstable once holes introduced by the Mn acceptors become compensated by electrons originating from As antisites or $\mathrm{Sn}$ donors. Here, as in most magnetic materials, antiferromagnetic superexchange dominates in the absence of the carriers. Surprisingly, however, the Mn magnetic moment increases only slightly as a function of compensation, from 4.2 to $4.3 \mu_{\mathrm{B}}$. This appears to constitute an additional hint that the holes reside rather on $\mathrm{p}$ than on $\mathrm{d}$ shells.

A destructive influence of antisites on the ferromagnetic phase in ordered $\mathrm{Ga}_{1-x} \mathrm{Mn}_{x}$ As alloys has been demonstrated also by Sanvito and Hill (2001). In another work devoted to effects of disorder, Schulthess and Butler (2001) compare results for a $\mathrm{Ga}_{1-x} \mathrm{Mn}_{x} \mathrm{As}$ supercell, $x=0.0625$, computed employing the Vienna $a b$ intio simulation package (VASP), with the outcome of CPA computations for an equivalent random alloy with $x=$ 0.05. According to the VASP data, the difference in total energies of antiferromagnetic and ferromagnetic ground states, normalized to one $\mathrm{Mn}$ atom, is $\Delta E=0.076 \mathrm{eV}$. For unspecified reason, this stability energy is appreciably smaller than the values of 0.15 and $0.12 \mathrm{eV}$ determined for the same Mn content by Sanvito and Hill (2001) and Zhao et al. (2001a), respectively. According to the CPA results, the disorder does not qualitatively affect the density of states but reduces $\Delta E$ to $0.045 \mathrm{eV}$ (Schulthess and Butler 2001). Exchange energies of various molecular clusters of $\mathrm{Cr}, \mathrm{Mn}$, and Fe embedded in zinc blende GaAs, GaN, and AlN have been computed within the LSDA by van Schlifgaarde and Mryasov (2001). According to this work, magnetic energy leads to a strong short-range attractive force between the magnetic dopants but whether the corresponding clustering is kinetically allowed at growth temperatures has not been assessed.

In all works referred to above LSDA or GGA have been employed. These approximations are known (Kotani 2000) to underestimate the on-site correlation effects for $3 \mathrm{~d}$ electrons in non-metallic solids. This means, according to the Anderson model of magnetic impurities, that magnitudes of $p-d$ hybridization and related interactions will be overestimated. For instance, the values of Néel temperatures in zinc blende Mn chalcogenides, evaluated within LSDA (Wei and Zunger 1993), are about two times larger than those determined experimentally. This discrepancy is removed in a computation scheme, known as LSDA+U, in which an external parametric potential is added, whose role is to displace the $d$ band, hence change the $p-d$

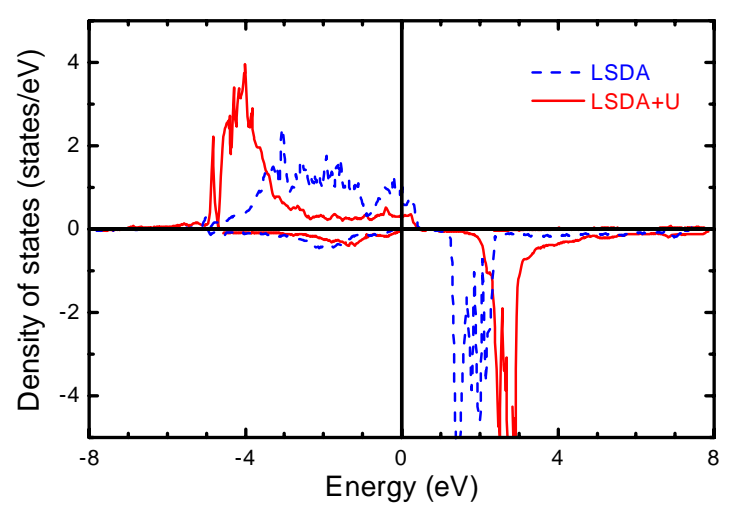

Fig. 23. The spin polarized Mn $3 \mathrm{~d}$ partial density of states of $\mathrm{Ga}_{0.938} \mathrm{Mn}_{0.063} \mathrm{As}$ from the LSDA and the LSDA+U (Park et al. 2000). coupling (Wei and Zunger, 1993). Figure 23 compares the partial density-of-states (DOS) for Mn d-electrons in an ordered $\mathrm{Ga}_{1-x} \mathrm{Mn}_{x}$ As alloy with $x=0.0625$ computed by Park et al. (2000) within LMTO-LSDA and LMTO-LSDA+U, respectively. In agreement with the Parmenter (1973) model, the parametric potential is characterized by two adjustable parameters, the correlation energy $U$ and Hund's intra-site exchange energy $J$. As expected, DOS from the LSDA+U model is sharper and located further off the Fermi energy than the LSDA d bands. Another interesting aspect of the theoretical results of Park et al. (2000) is the observation that the coupling of two Mn spins changes from ferromagnetic to antiferromagnetic when the distance between the pair increases, a behavior reminiscent of the Ruderman-Kittel-Kasuya-Yosida oscillations. It would be interesting to check the stability of the ferromagnetic phase and the magnitude of $N_{0} \beta$ within the LSDA+U model.

It is tempting to compare the computed DOS with photoemission spectra. Certainly, DOS obtained within the LSDA+U model agrees better with photoemission results (Okabayashi et al. 1998, 1999, 2001a, 2001b), though a direct comparison is hampered by the influence of electron-hole correlation and multiplet effects on the form of excitation spectra (Mizokawa and Fujimori 1993). According to the LSDA+U model, the Mn occupancy increases to 5.4, and the participation of $d$ states in the wave function of holes near the Fermi energy is reduced compared to the LSDA results (Park et al. 2000). The occupation number in excess of 5 is directly corroborated by x-ray magnetic circular dichroism (XMCD) studies (Ohldag et al. 2000, Ueda et al. 2001), and also, in a model dependent way, by photoemission experiments of Okabayashi et al. (1998, 1999), which lead to the value $5.3 \pm$ 0.1 , as discussed in Sec. 4. At the same time, the local magnetic moment, which amounts $4.1 \mu_{\mathrm{B}}$ in LSDA, increases to $4.4 \mu_{\mathrm{B}}$ in LSDA+U (Park et al. 2000). The latter compares favorably with the value $4.6 \mu_{\mathrm{B}}$ determined by fitting XMCD spectra (Ohldag et al. 2000). According to results presented in Sec. 5, the saturation values of the magnetization point to a magnitude of the magnetic moment between 4 and $5 \mu_{\mathrm{B}}$, its more accurate determination being precluded by difficulties in the evaluation of the Mn content $x$.

\subsection{Parameterized Hamiltonians}

Experimental and theoretical results presented in the previous sections make it possible to sketch a picture of interplay between electronic and magnetic properties in (Ga,Mn)As. This magnetic semiconductor can be classified as a charge transfer insulator, in which the high spin state, $S=5 / 2$, is stabilized by positive values of energies required to transfer an electron either from the Mn 3d shell to the Fermi level or vice versa. Since Mn atoms are divalent in the $S=5 / 2$ configuration, they act as effective mass acceptors when they substitute for trivalent Ga. The corresponding energy level should not be confused with the Mn 3d shells. At the same time, it is important to realize that the quasi-atomic Woodbury-Ludwig description of magnetic $d$ orbitals is by no means valid in the case of doping by $3 \mathrm{~d}$ transition metals. Actually, the position and origin of both band and 3d-like local states are determined-to a large extend-by their strong hybridization (Zunger 1986). In particular, the hybridization accounts for a 
large splitting between $t_{2 g}$ and $e_{g}$ components. Moreover, it leads to an admixture of the $\mathrm{d}^{6}$ configuration to the wave function of the occupied states visible in XMCD and $\mathrm{x}$-ray photoemission spectra (XPS), as discussed above. Furthermore, the p-d interaction tends to renormalize the energy of anion p-type orbitals adjacent to $\mathrm{Mn}$ atoms. This will contribute to the "chemical shift" of Mn acceptors ionization energy in GaAs:Mn and to the valence band off-set in (Ga,Mn)As. Importantly, a part of the p-d interaction is spin-dependent, which according to the Schrieffer-Wolf transformation, leads to a Kondo-like coupling between effective mass carriers and localized spins (Kacman 2001).

In view of the above discussion, the one-carrier effective mass Hamiltonian describing the interaction with a $\mathrm{Mn}$ atom located at $\boldsymbol{R}_{\mathrm{i}}$ assumes the form,

$$
H_{i}=H_{\mathrm{o}}(\boldsymbol{p})+V_{\mathrm{C}}\left(\boldsymbol{r}-\boldsymbol{R}_{i}\right)+V_{\mathrm{s}}\left(\boldsymbol{r}-\boldsymbol{R}_{i}\right)-I\left(\boldsymbol{r}-\boldsymbol{R}_{i}\right) \boldsymbol{s} \boldsymbol{S}_{i},
$$

where $H_{0}(\boldsymbol{p})$ is the $\boldsymbol{k} \cdot \boldsymbol{p}$ Hamiltonian for the crystal structure in question, $V_{\mathrm{C}}$ and $V_{\mathrm{s}}$ describe the Coulomb and short-range "chemical" part of the potential introduced by the Mn ion, respectively, and $I$ is the short-range exchange operator. It appears that as long as the magnetic ion occupies the tetrahedral position (so that there is no, e. g. static Jahn-Teller distortion), the Kondo form of the spin dependent interaction is valid even for magnetic ions with non-zero orbital momentum (Kacman 2001). However, the orbital momentum, and the associated spin-orbit interaction will affect the relation between magnetization $\boldsymbol{M}$ and expectation value of spin operator $\boldsymbol{S}$. At the same time, in order to describe correctly the influence of the exchange interaction on the effective mass states, it is essential to take the spin-orbit interaction into account in the $\boldsymbol{k} \cdot \boldsymbol{p}$ Hamiltonian $H_{0}(\boldsymbol{p})$.

It is convenient to replace, in the spirit of the Kohn-Luttinger effective mass theory for the valence subbands, short range potentials by appropriate matrix elements $W$ and $\beta$ according to $W=\left\langle X\left|V_{\mathrm{s}}\right| X>\right.$ and $\beta=\langle X|I| X>$, where $X$ denotes a $p_{x}$-like component of the Bloch wave functions for the $\Gamma_{8}$ point of the Brillouin zone. The parameter $\beta$ is the familiar exchange integral, whereas $W$ describes the valence band off set disregarding long-range Coulomb effects. Such a parameterized Hamiltonian (6) and its variants constitute a starting point for a number of ferromagnetism models, which will be outlined in this section. First, however, available information on the values of $W$ and $\beta$ is summarized.

As already mentioned, theoretical results of Sanvito et al. (2001) leads to $N_{0} \beta \approx-4.5 \mathrm{eV}$ and $W$ close to zero. Experimental source of information on $\beta$ and $W$ are parameters serving to describe photoemission and XMCD results. Both XPS (Okabayashi et al. 1998), and resonance photoemission spectra (Okabayashi et al. 1999) can be described with the same set of charge transfer energies in the cluster model (Mizokawa and Fujimori 1993), which result in $N_{0} \beta=-1.2 \pm 0.2 \mathrm{eV}$ for $\mathrm{Ga}_{1-x} \mathrm{Mn}_{x}$ As with $x=0.074$ (Okabayashi et al. 1998). At the same time, the values of the transfer energies inserted into the formula of Hass (1991) imply that the contribution of the p-d interaction to $\left|N_{0} W\right|$ is below $1 \mathrm{eV}$. However, the application of the model of Mizokawa and Fujimori (1993) to XMCD data leads to somewhat different values of the charge transfer energies, from which $N_{0} \beta \approx-0.34 \mathrm{eV}$ and $W_{\mathrm{pd}}>0$ in
$\mathrm{Ga}_{1-x} \mathrm{Mn}_{x}$ As with $x=0.025$ (Ueda et al. 2001).

Bhattacharjee and Benoit à la Guillaume (2000) adopted the Hamiltonian (6) in order to describe experimental values of spin-flip and ionization energies of holes localized on $\mathrm{Mn}$ acceptors in the case of weak doping, $x<0.1 \%$. Their results, obtained by employing the Baldereschi-Lipari effective mass theory, lead to $N_{0} \beta \approx-0.9 \mathrm{eV}$. However, for the assumed shape of the acceptor wave function, the magnitude of $\left|N_{0} \beta\right|$ would, presumably, be about two times smaller when taking a more recent value of the spin flip energy, as determined by Sapega et al. (2000, 2001). According to Bhattacharjee and Benoit à la Guillaume (2000), a positive value of $N_{0} W$ having a magnitude larger than $\left|N_{0} \beta\right|$ is necessary in order to explain the "chemical shift" of the Mn acceptor. The positive sign of $W$ is consistent with the existence of a barrier for the hole injection from (Ga,Mn)As to GaAs in p-i-n diodes (Arata et al. 2001). It has been concluded, by comparing results for three kinds of $\mathrm{p}-\mathrm{i}$ heterojunctions: (Ga,Mn)As/GaAs, GaAs:Be/GaAs, and $\mathrm{GaAs}: \mathrm{Be} /(\mathrm{Al}, \mathrm{Ga}) \mathrm{As}$ that the barrier height in $(\mathrm{Ga}, \mathrm{Mn}) \mathrm{As} / \mathrm{GaAs}$ is too large to be entirely assigned to band gap narrowing by many body effects within the carrier liquid. A barrier height of $100 \mathrm{meV}$ for $x=0.05$, would imply $N_{0} W=2 \mathrm{eV}$ in the virtual crystal approximation. Such a band off-set is consistent with a reduction of the energy gap in ( $\mathrm{Ga}, \mathrm{Mn})$ As comparing to GaAs, noted by Dietl et al. (2001c) and Szczytko et al. (2001b) when analyzing optical transmission data of Szczytko et al. (1999b) and Beschoten et al. (1999). Importantly, assuming a value of $N_{0} \beta=-1.2 \mathrm{eV}$, a consistent account of the MCD spectra of Beschoten et al. (1999) is possible within the $\boldsymbol{k} \cdot \boldsymbol{p}$ model of the valence band (Dietl et al. 2001c). More recently, Szczytko et al. (2001b) by taking the effect of disorder on selection rules into account obtained an accurate description of their magneto-transmission results (Szczytko et al. 1999b) with $N_{0} \beta$ $=-1.0 \mathrm{eV}$. Finally, we recall that a description of the temperature and field dependence of the resistance in terms of spin-disorder scattering yielded $\left|N_{0} \beta\right|$ of $1.5 \pm 0.2 \mathrm{eV}$ (Omiya et al. 2000), assuming a simple structure of the valence band and neglecting the $\boldsymbol{q}$-dependence of the magnetic susceptibility.

The body of findings presented above demonstrates that owing to the $\mathrm{p}-\mathrm{d}$ hybridization there exists an antiferromagnetic coupling between the hole and $\mathrm{Mn}$ spins in (Ga,Mn)As. If described in terms of the Kondo Hamiltonian, the magnitude of the corresponding exchange energy is, presumably, of the order of $-1 \mathrm{eV}, N_{0} \beta \approx-1.0 \mathrm{eV}$. Furthermore, in the GaAs/(Ga,Mn)As heterojunction, the valence band edge of $(\mathrm{Ga}, \mathrm{Mn}) \mathrm{As}$ resides higher than in GaAs, $N_{0} W>0$, so that the potential $V_{\mathrm{s}}$ in eq. (6) is attractive for the holes. However, to what extent this band offset is controlled by the $\mathrm{p}$-d hybridization and what is its exact magnitude is unknown at present.

\subsection{Hole states and hole mediated exchange interactions}

It is now well established that in the absence of free carriers the dominant exchange mechanism is the superexchange in zinc blende magnetic semiconductors. This mechanism leads to antiferromagnetic interactions, except perhaps for some Cr-based compounds, for which a ferromagnetic coupling is theoretically predicted (Blinowski et al. 1996). Remarkably, owing to the large exchange energy $\left|N_{0} \beta\right|$ and the high density of states, the hole-mediated ferromagnetic 
exchange interaction can overcome antiferromagnetic superexchange (Dietl et al. 1997). Indeed, as already emphasized, the presence of holes is essential for the existence of the ferromagnetic order in Mn-based semiconductors. The case of III-V magnetic semiconductors is particularly fortunate as $\mathrm{Mn}$ atoms act as acceptors. It should be recalled at this point that electronic states in doped semiconductors undergo dramatic changes as a function of the impurity concentration (Belitz and Kirkpatrick 1994, Edwards and Rao 1995). Hence, the hole states, and possibly hole-mediated exchange mechanisms, may a priori undergo dramatic changes as function of the Mn content $x$ and the concentration of compensating donors, $N_{\mathrm{D}}$ in III-V magnetic semiconductors.

The evolution of electronic states in doped semiconductors is governed by the ratio of the average distance between the carriers $r_{\mathrm{c}}$ to the effective impurity Bohr radius $a_{\mathrm{B}}$, determined by both Coulomb and short-range potentials of eq. (6). In the case of the holes in $(\mathrm{Ga}, \mathrm{Mn}) \mathrm{As}, r_{\mathrm{c}}=(3 / 4 \pi p)^{1 / 3}, p=x N_{0}-N_{\mathrm{D}}$, and $a_{\mathrm{B}} \approx 0.78 \mathrm{~nm}$ (Bhatt and Berciu 2001). In the range of small impurity concentrations, $r_{\mathrm{c}}>a_{\mathrm{B}}$, the holes are tightly bound to acceptors. Hence, the conductivity vanishes in the limit of zero temperature. At non-zero temperatures, the charge transport proceeds either via phonon-assisted hopping between occupied and empty acceptors or by means of thermal activation from the acceptor levels to the valence band. In a pioneering work Pashitskii and Ryabchenko (1979) evaluated the strength of exchange interactions between localized spins mediated by band carriers thermally activated from impurity levels. More recently, Wolff et al. (1996) considered carriers localized on impurities and forming bound magnetic polarons (BMP). It was found that there exists a range of parameters, in which the coupling between the BMP is ferromagnetic. This idea was further explored by Bhatt and Wan (1999), who examined by Monte Carlo simulations properties of a ferromagnetic phase transition driven by the interactions between BMP.

Two other groups noted that a long-range exchange interaction between $\mathrm{Mn}$ spins can be mediated by holes undergoing quantum hoping from the Mn-derived impurity states to the extended valence band states. Inoue et al. (2000) adopted the Slater-Koster approach, well known in the physics of resonant states, for the case of two magnetic impurities. It has been found, by a model calculation, that the pairs of Mn spins coupled to the valence band states have a lower energy in the ferromagnetic than in the antiferromagnetic configuration. Litvinov and Dugajev (2001) suggested than the ferromagnetic spin-spin interaction can originate from virtual excitations between the acceptor-like impurity level and the valence band, a variant of the Bloembergen-Rowland indirect exchange mechanism. They evaluated Curie temperatures by using a formula, derived originally for excitations between valence and conduction bands, without proving its correctness for the case in question.

With the increase of the net acceptor concentration, the impurity band merges with the valence band. For $r_{\mathrm{c}}<<a_{\mathrm{B}}$, the holes reside in the band, and their quasi-free propagation is only occasionally perturbed by scattering of Mn (eq. (6)) and other defect potentials, whose long-range Coulomb part is screened by the carrier liquid. Here, the celebrated Ruderman-Kittel-Kasuya-Yosida (RKKY) mechanism, driven by intraband virtual excitations, is expected to dominate. In the context of III-V magnetic semiconductors, this mechanism was discussed by Gummich and da Cunha Lima (1990) and Matsukura et al. (1998b). At the same time, Dietl et al. (1997) demonstrated the equivalence of the RKKY and Zener (1951a, 1951c) models, at least on the level of the mean-field and continuous medium approximations. However, with no doubts, beyond those approximations such equivalence can be questioned (Semenov and Ryabchenko 2001).

Within the Zener (1951a, 1951c) approach, and its nuclear spin variant (Fröhlich and Nabarro 1940), the degree of spin ordering, $\boldsymbol{M}_{q}$, at given temperature $T$ is found by minimizing the total free energy of the spin and carrier subsystems, $F\left[\boldsymbol{M}_{q}\right]$. Here $\boldsymbol{M}_{\boldsymbol{q}}$ denotes the Fourier components of localized spin magnetization $\boldsymbol{M}(r)$, so that the minimum of $F\left[\boldsymbol{M}_{\boldsymbol{q}}\right]$ for $\boldsymbol{M}_{\boldsymbol{q}=\mathbf{0}} \neq 0$ implies the ferromagnetic order. In general, however, other ground states, such as non-collinear structures or spin-density waves, described by $\boldsymbol{M}_{\boldsymbol{q} \neq 0}$ have to be considered (Dietl et al. 1999). This is a rather versatile scheme, to which carrier correlation and confinement (Dietl et al. 1997, 1999, Haury 1997, Jungwirth et al. 1998, Lee et al. 1999, Fernández-Rossier and Sham 2001), $\boldsymbol{k} \cdot \boldsymbol{p}$ and spin-orbit couplings (Dietl et al. 2000, 2001c, Abolfath et al. 2001, Fernández-Rossier and Sham 2001) as well as disorder and antiferromagnetic interactions (Dietl et al. 1997, Kossacki et al. 2000) can be introduced in a controlled way, and within which a quantitative comparison of experimental and theoretical results is possible (Dietl et al. 2001a, 2001c, Ferrand et al. 2001).

In view of the above discussion the question arises whether the hole-mediated ferromagnetism appears in the insulator or in the metallic phase. It is well established that the metal-insulator transition (MIT) occurs at $r_{\mathrm{c}} \approx 2.4 a_{\mathrm{B}}$ in doped non-magnetic semiconductors (Edwards and Sienko 1978). According to this criterion one gets the critical hole concentration $p_{\mathrm{c}}=4 \times 10^{19}$ $\mathrm{cm}^{-3}$ for $a_{\mathrm{B}}=0.78 \mathrm{~nm}$. Experimentally, the MIT occurs at about $3.5 \%$ of $\mathrm{Mn}$ in (Ga,Mn)As, i.e., for $N_{0} x=7 \times 10^{20} \mathrm{~cm}^{-3}$ (Oiwa et al. 1997, Matsukura et al. 1998b, Katsumoto et al. 1998). A large difference between these two values is presumably caused by the compensation (discussed above) as well as by the enhancement of localization by the sp-d exchange scattering (Dietl 1994). The latter is documented in (Ga,Mn)As by the presence of negative magnetoresistance and associated insulator-to-metal transition driven by the magnetic field (Katsumoto et al. 1998). In addition to the MIT at $x \approx 0.035$, the reentrant insulator phase is observed for $x>0.06$ (Matsukura et al. 1998b), as discussed in Sec. 6. Presumably, a self-compensation mechanism is involved but no microscopic model has been proposed so far.

Perhaps, the most intriguing property of the materials in question is that the ferromagnetism is observed on the both sides of MIT (Oiwa et al. 1997, Matsukura et al. 1998b). It is, therefore, interesting to contemplate the nature of electronic states in the vicinity of the MIT in doped semiconductors. Obviously, the random spatial distribution of acceptor and donor centers gives rise to strong spatial fluctuations in the carrier density and states characteristics. According to the phenomenological two-fluid model there exist two kinds of relevant states (Paalanen and Bhatt 1991). The first are strongly localized and thus singly occupied states associated with the attractive potential of a single majority impurity. The strongly localized carriers barely contribute to the conduction process. 
However, they produce a Curie-like component in the magnetic susceptibility and give rise to the presence of BMP in magnetic semiconductors. Obviously, the impurity-like states dominate deeply in the insulating phase but their presence is noticeable also in the metallic phase (Paalanen and Bhatt 1991, Głód et al. 1994). The second pool of states determines the conductivity, so that properties of these states are described by the scaling theory of MIT. Accordingly, the corresponding localization radius $\xi$ is rather controlled by interference of multi-scattering processes than by the attractive potential of a single impurity. Thus, $\xi$ of these weakly localized states is significantly larger than $a_{\mathrm{B}}$, and diverges on approaching the MIT from the insulator side. It is worth noting that such a two-fluid model is consistent with a.c. conductivity studies (Nagai et al. 2001), which show the coexistence of weakly and strongly localized states near the MIT in (Ga,Mn)As. Furthermore, the merging of impurity and band states in this range is substantiated by angle-resolved photoemission spectra in the same system (Okabayashi et al. 2001a, 2001b).

In order to tell the dominant mechanism accounting for the existence of long-range spin order in ferromagnetic semiconductors it is instructive to trace the evolution of their magnetic properties on crossing the MIT. Remarkably, in contrast to rather strong changes of resistivity, the evolution of magnetic properties is gradual. This substantiates the notion that thermodynamic properties do not exhibit any critical behavior at MIT as they are insensitive to large-scale characteristics of the wave functions. Importantly, the values of the Curie temperature are found to grow with the degree of the material metallicity (Matsukura et al. 1998b, Katsumoto et al. 2001, Potashnik et al. 2001). Moreover, the examination of the magnetization as a function of temperature and magnetic field indicates that virtually all $\mathrm{Mn}$ spins contribute to ferromagnetic order in the most metallic samples (Oiwa et al. 1997, Matsukura et al. 1998b, Potashnik et al. 2001). However, on crossing the MIT (by lowering $x$ ), the relative concentration of ferromagnetically coupled spins decreases substantially. According to XMCD results of Ohldag et al. (2000), about $10 \%$ of the $\mathrm{Mn}$ spins is involved in ferromagnetism of $\mathrm{Ga}_{1-x} \mathrm{Mn}_{x}$ As with $x=2 \%$. Also ferromagnetic resonance studies (Szczytko et al. 1999b) and direct magnetization measurements demonstrate that only a part of the spins contribute to spontaneous magnetization, while the alignment process of the remaining moments occurs according to a Brillouin function for a weakly interacting spin system (Oiwa et al. 1997). Remarkably, the anomalous Hall effect reveals clearly the presence of the first component but hardly points to the existence of any loose spins (Matsukura et al. 1998b).

The above findings indicate that Mn spins in the regions visited by itinerant holes are coupled ferromagnetically. These holes set long-range ferromagnetic correlations between the Mn spins, including those contributing to BMP that are formed around singly occupied local states. Obviously, the ferromagnetic portion of the material, and thus the magnitude of the spontaneous magnetization, grows with the dopant concentration, attaining $100 \%$ in the metallic phase. Such a trend is confirmed by the available data, as discussed above. Thus, the delocalized or weakly localized holes are responsible for ferromagnetic correlation in (Ga,Mn)As (Dietl et al. 2000). At the same time, mechanisms that involve strongly localized states, such as excitations from impurity levels or a direct coupling between BMP, appear to be of lesser importance.

\subsection{Mean-field Zener model and its application to (Ga,Mn)As}

In this section, theoretical foundations and application of the mean-field Zener (1951a, 1951c) model to III-V magnetic semiconductors are discussed in some detail. The capabilities of the model to describe various magnetic properties of $(\mathrm{Ga}, \mathrm{Mn}) \mathrm{As}$ are presented, too. In the final part, limitations of the model and its numerous refinements put recently forward are discussed.

As already mentioned, it is convenient to apply the Zener model (1951a, 1951c) by introducing the Ginzburg-Landau functional $F[\boldsymbol{M}(\boldsymbol{r})]$ of the free energy density (Ma 1976), where $\boldsymbol{M}(\boldsymbol{r})$ denotes local magnetization of the Mn spins. The choice of $\boldsymbol{M}(\boldsymbol{r})$ as the order parameter means that the spins are treated as classical vectors, and that spatial disorder inherent to magnetic alloys is neglected. In the case of magnetic semiconductors $F[\boldsymbol{M}(\boldsymbol{r})]$ consists of two terms, $F[\boldsymbol{M}(\boldsymbol{r})]=$ $F_{\mathrm{S}}[\boldsymbol{M}(\boldsymbol{r})]+F_{\mathrm{c}}[\boldsymbol{M}(\boldsymbol{r})]$, which describe, for a given magnetization profile $\boldsymbol{M}(\boldsymbol{r})$, the free energy densities of the Mn spins in the absence of any carriers and of the carriers in the presence of the Mn spins, respectively (Leroux-Hugon 1973, Dietl 1994). A visible asymmetry in the treatment of the carries and of the spins corresponds to an adiabatic approximation, the dynamics of the spins in the absence of the carriers being assumed to be much slower than that of the carriers. Furthermore, in the spirit of the virtual-crystal and molecular-field approximations, the classical continuous field $\boldsymbol{M}(\boldsymbol{r})$ controls the effect of the spins upon the carriers. Now, the system thermodynamics is described by the partition function $Z$, which can be obtained by a functional integral of the Boltzmann factor $\exp \left(-\int \mathrm{d} \boldsymbol{r} F[\boldsymbol{M}(\boldsymbol{r})] / k_{\mathrm{B}} T\right)$ over all magnetization profiles. In the mean-field approximation (MFA), a term corresponding to the minimum of $F[\boldsymbol{M}(\boldsymbol{r})]$ is assumed to determine $Z$ with a sufficient accuracy.

If energetics is dominated by a spatially uniform magnetization $\boldsymbol{M}$, the spin part of the free energy density in the magnetic field $\boldsymbol{H}$ can be written in the form

$$
F_{S}[\vec{M}]=\int_{0}^{\vec{M}} d \vec{M}_{o} \vec{h}\left(\vec{M}_{o}\right)-\vec{M} \vec{H}
$$

Here, $\boldsymbol{h}\left(\boldsymbol{M}_{\mathrm{o}}\right)$ denotes the inverse function to $\boldsymbol{M}_{\mathrm{o}}(\boldsymbol{h})$, where $\boldsymbol{M}_{\mathrm{o}}$ is the macroscopic magnetization of the spins in the absence of carriers at a field $\boldsymbol{h}$ and temperature $T$. It is usually possible to parameterize $\boldsymbol{M}_{\mathrm{o}}(\boldsymbol{h})$ by the Brillouin function $\mathrm{B}_{S}$ according to

$$
\boldsymbol{M}_{\mathrm{o}}(\boldsymbol{h})=x_{\mathrm{eff}} N_{\mathrm{o}} g \mu_{\mathrm{B}} S B_{S}\left[g \mu_{\mathrm{B}} \boldsymbol{h} / k_{\mathrm{B}}\left(T+T_{\mathrm{AF}}\right)\right]
$$

where two empirical parameters, the effective spin concentration $x_{\text {eff }} N_{\mathrm{o}}<x N_{\mathrm{o}}$ and the temperature $T_{\text {eff }}>T$, take the presence of the short-range superexchange antiferromagnetic interactions into account (Gaj et al. 1979). The dependencies $x_{\mathrm{eff}}(x)$ and $T_{\mathrm{AF}}(x)$ are known for II-VI DMS compounds. However, as argued by Dietl et al. (2000, 2001c), the antiferromagnetic short-range interaction is overcompensated by the ferromagnetic double exchange coupling in the case of III-V DMS. According to the 
two fluids model introduced in Sec. 8.3, a part of the carriers is trapped on strongly localized impurity states, and thus forms BMP. To gain the Coulomb energy, the singly occupied local states are preferentially formed around close pairs of ionized acceptors. In the case of III-V materials, one hole localized at two Mn ions generates, via Zener's (1951b) double exchange, a strong ferromagnetic coupling that overcompensates the intrinsic antiferromagnetic interaction (Blinowski et al. 1997). Accordingly, $x_{\text {eff }} \approx x$ and $T_{\text {eff }} \approx T$. By contrast, in II-VI compounds in which acceptor cores do not carry any spin, and the degree of compensation is low, BMP are not preferentially formed around Mn pairs, so that the close pairs remain antiferromagnetically aligned. The presence of a competition between the ferromagnetic and antiferromagnetic interactions in p-type II-VI DMS, and its absence in Mn-based III-V materials, constitutes the important difference between those two families of magnetic semiconductors.

It is clear from eqs. (7) and (8) that $F_{\mathrm{S}}[\boldsymbol{M}]$ monotonously increases with $|\boldsymbol{M}|$, so that-as expected-the minimum of $F_{\mathrm{S}}[\boldsymbol{M}]$ corresponds to $\boldsymbol{M}=0$, for which the spin entropy attains the highest value. It is convenient to introduce the spin susceptibility $\tilde{\chi}_{S}$ related to the magnetic susceptibility $\chi_{o}$ of the spins according to $\tilde{\chi}_{S}=\left(\mathrm{g} \mu_{\mathrm{B}}\right)^{2} \chi_{o}$. In the limit, where $M_{o}(T, h)=\chi_{o}(T) h$,

$$
F_{S}[\vec{M}]=\vec{M}^{2} /\left[2\left(g \mu_{B}\right)^{2} \tilde{\chi}_{S}(T)\right]
$$

which shows that the increase of $F_{\mathrm{S}}$ with $M$ slows down with lowering temperature. In contrast to $F_{\mathrm{S}}[\boldsymbol{M}]$, owing to Zeeman splitting of the bands imposed by the sp-d exchange interaction, the energy of the carriers, and thus $F_{\mathrm{c}}[\boldsymbol{M}]$, decreases with $|\boldsymbol{M}|$. Accordingly, a minimum of $F[\boldsymbol{M}]$ at non-zero $\boldsymbol{M}$ may develop in $H=0$ at sufficiently low temperatures.

In order to take into account the complex structure of the valence band, Dietl et al. $(2000,2001 \mathrm{c})$ and Abolfath et al. (2001) have computed hole energies by diagonalizing the 6x6 $\boldsymbol{k} \cdot \boldsymbol{p}$ Luttinger matrix together with the $\mathrm{p}-\mathrm{d}$ exchange contribution taken in the virtual crystal and molecular field approximation,

$$
H_{\mathrm{pd}}=\beta \boldsymbol{s} \boldsymbol{M} / g \mu_{\mathrm{B}}
$$

This term leads to spin splittings of the valence subbands, whose magnitudes - owing to the spin-orbit coupling - depends on the hole wave vectors in a complex way even for spatially uniform magnetization, as shown in Fig. 24. It would be technically difficult to incorporate such effects to the RKKY model, as the spin-orbit coupling leads to non-scalar terms in the spin-spin Hamiltonian. At the same time, the indirect exchange associated with the virtual spin excitations between the valence subbands, the Bloembergen-Rowland mechanism (Dietl 1994, Kacman 2001), is automatically included. The model allows for biaxial strain (Dietl et al. 2000, 2001c, Abolfath et al. 2001), confinement (Fernández-Rossier and Sham 2001), and is developed for both zinc blende and wurzite materials (Dietl et al. 2000, 2001c). Furthermore, Dietl et al. (2001c) take into consideration the direct influence of the magnetic field on the hole spectrum. The carrier-carrier spin correlation is described by introducing a Fermi-liquid-like parameter $A_{\mathrm{F}}$, which enlarges the Pauli susceptibility of the hole liquid (Altshuler and Aronov 1985, Dietl et al. 1997). No disorder effects are taken into account on the grounds that their influence on thermodynamic properties is relatively weak.

Having the hole energies, the Helmholtz free energy density $F_{\mathrm{c}}[\boldsymbol{M}]$ can be evaluated according to the standard procedure for the Fermi gas. By minimizing $F[\boldsymbol{M}]=F_{\mathrm{S}}[\boldsymbol{M}]+$ $F_{\mathrm{c}}[\boldsymbol{M}]$ with respect to $\boldsymbol{M}$ at given $T, \boldsymbol{H}$, and hole concentration $p$, one obtains $\boldsymbol{M}(T, \boldsymbol{H})$ as a solution of the mean-field equation,

$\boldsymbol{M}(T, \boldsymbol{H})=x_{\mathrm{eff}} N_{\mathrm{o}} g \mu_{\mathrm{B}} S B_{S}\left[g \mu_{\mathrm{B}}\left(-\partial F_{\mathrm{c}}[\boldsymbol{M}] / \partial \boldsymbol{M}+\boldsymbol{H}\right) / k_{\mathrm{B}}\left(T+T_{\mathrm{AF}}\right)\right]$,

where peculiarities of the valence band structure, such as the presence of various hole subbands, anisotropy, and spin-orbit coupling, are hidden in $F_{\mathrm{c}}[\boldsymbol{M}]$. Near the Curie temperature $T_{\mathrm{C}}$ and at $H=0$, where $M$ is small, we expect $F_{\mathrm{c}}[\boldsymbol{M}]-F_{\mathrm{c}}[0] \sim \boldsymbol{M}^{2}$. It is convenient to parameterize this dependence by a generalized carrier spin susceptibility $\tilde{\chi}_{c}$, which is related to the magnetic susceptibility of the carrier liquid according to $\chi=$ $A_{\mathrm{F}}\left(g^{*} \mu_{\mathrm{B}}\right)^{2} \tilde{\chi}_{c}$. In terms of $\tilde{\chi}_{c}$,

$$
F_{\mathrm{c}}[\boldsymbol{M}]=F_{\mathrm{c}}[0]-A_{\mathrm{F}} \tilde{\chi}_{c} \beta^{2} \boldsymbol{M}^{2} / 2\left(g \mu_{\mathrm{B}}\right)^{2} .
$$

By expanding $B_{\mathrm{S}}(\boldsymbol{M})$ one arrives at the well-known form for the mean-field value of $T_{\mathrm{C}}$ (Dietl et al. 1997, Jungwirth et al. 1999)

$$
T_{\mathrm{C}}=x_{\mathrm{eff}} N_{\mathrm{o}} S(S+1) A_{\mathrm{F}} \tilde{\chi}_{c}\left(T_{\mathrm{C}}\right) \beta^{2} / 3 k_{\mathrm{B}}-T_{\mathrm{AF}} .
$$

For a strongly degenerate carrier liquid $\left|\mathcal{E}_{\mathrm{F}}\right| / k_{\mathrm{B}} T \gg 1$, as well as neglecting the spin-orbit interaction, $\tilde{\chi}_{c}=\rho / 4$, where $\rho$ is the total density-of-states for intra-band charge excitations, which in the $3 \mathrm{D}$ case is given by $\rho=m^{*}{ }_{\mathrm{Dos}} k_{\mathrm{F}} / \pi^{2} \hbar^{2}$. In general, however, $\tilde{\chi}_{c}$ has to be determined numerically by computing $F_{\mathrm{c}}[\boldsymbol{M}]$. Large magnitudes of both density of states and exchange integral specific to the valence band make $T_{\mathrm{C}}$ much higher in p-type than in n-type materials with a comparable carrier concentration.

The above reasoning can easily be generalized to the case of a phase transition to a spatially modulated ground state,

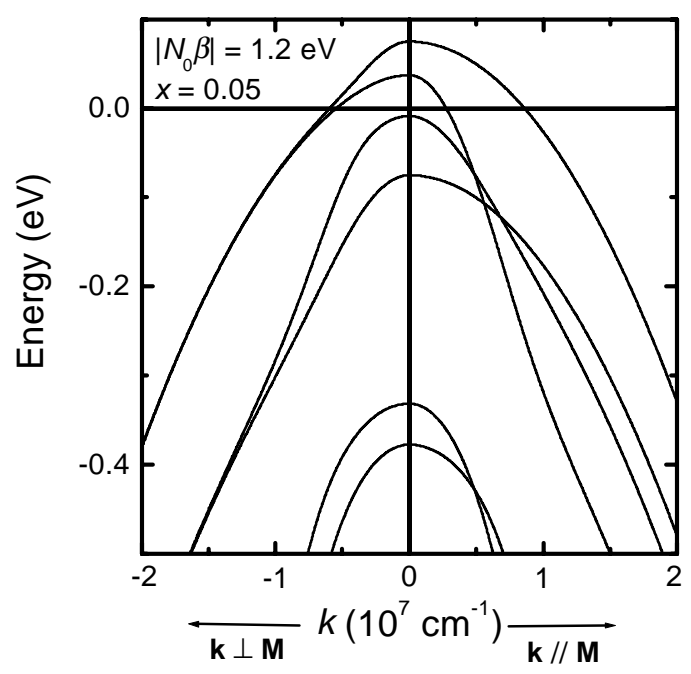

Fig. 24. The computed valence band dispersion $E(\boldsymbol{k})$ computed from the $6 \times 6$ Luttinger model for the wave vector parallel and perpendicular to the $\mathrm{Mn}$ spin magnetization in (Ga,Mn)As, assuming that the spin splitting of the heavy-hole band at the $\Gamma$ point is $0.15 \mathrm{eV}$. 
characterized by non-zero magnetization $\boldsymbol{M}_{\boldsymbol{q}}$. The corresponding mean-field value of the ordering temperature $T_{\mathrm{c}}(\boldsymbol{q})$ is given by the solution of the equation (Dietl et al. 1999)

$$
A_{F}(\vec{q}) \beta^{2} \tilde{\chi}_{c}(\vec{q}, T) \tilde{\chi}_{S}(\vec{q}, T)=1,
$$

where the carrier spin susceptibility can be determined from the standard linear-response expression,

$$
\tilde{\chi}_{c}^{(i i)}(\vec{q}, T)=\frac{2}{V} \sum_{\vec{k}, n, n^{\prime}}^{\prime} \frac{\left|\left\langle u_{\vec{k}+\vec{q}}^{\left(n^{\prime}\right)}\left|s_{i}\right| u_{\vec{k}}^{(n)}\right\rangle\right|^{2} f_{\vec{k}}^{(n)}\left(1-f_{\vec{k}+\vec{q}}^{\left(n^{\prime}\right)}\right)}{E_{\vec{k}+\vec{q}}^{\left(n^{\prime}\right)}-E_{\vec{k}}^{(n)}}
$$

Here $u_{\boldsymbol{k}}^{(n)}, f_{\boldsymbol{k}}^{(n)}$, and $E_{\boldsymbol{k}}^{(n)}$ are the periodic part of the Bloch function, energy and Fermi-Dirac distribution functions for the $n$-th carrier spin subband. In the case of cubic symmetry, the susceptibility tensor is isotropic, $\tilde{\chi}_{c}{ }^{(i j)}=\tilde{\chi}_{c} \delta_{i j}$. It has been checked within the $4 \times 4$ Luttinger model that the values of $T_{\mathrm{C}}$, determined from eqs. (13) and (12), which do not involve explicitly $u_{\boldsymbol{k}}^{(n)}$, and from eqs. (14) and (15) in the limit $q \rightarrow 0$, are identical (Ferrand et al. 2001). Such a comparison demonstrates that almost $30 \%$ of the contribution to $T_{\mathrm{C}}$ originates from interband polarization, i.e. from virtual transitions between heavy and light hole subbands.

It is possible to extend the above approach to the case of low dimensional structures (Dietl et al. 1997, 1999, Haury et al. 1997, Lee et al. 2000, Fernández-Rossier and Sham 2001). If the carriers occupy one electric subband, the mean-field value of ordering temperature $T_{\mathrm{c}}(\boldsymbol{q})$ is given by the formula that generalizes eq. (13) (Dietl et al. 1999)

$$
A_{F}(\vec{q}) \beta^{2} \tilde{\chi}_{c}(\vec{q}, T) \int \mathrm{d} \vec{\zeta}\left|\varphi_{o}(\vec{\zeta})\right|^{4} \tilde{\chi}_{S}(\vec{\zeta}, \vec{q}, T)=1,
$$

where $\varphi_{o}(\vec{\zeta})$ is the envelope function of the occupied subband in the confining potential, $\vec{\varsigma}$ and $\vec{q}$ are vectors in the $3-d$ and $d$ dimensional space, respectively.

We now turn to the ferromagnetic phase, $T<T_{\mathrm{C}}$. Here in addition to $\boldsymbol{M}(T, \boldsymbol{H})$, the evaluation of $F_{\mathrm{c}}[\boldsymbol{M}]$ makes it possible to determine the energy density of magnetic anisotropy, $K$ (Dietl et al. 2000, 2001c, Abolfath et al. 2001) as well as the hole spin polarization $\boldsymbol{P}=2 g \mu_{\mathrm{B}}\left(\partial F_{\mathrm{c}}[\boldsymbol{M}] / \partial \boldsymbol{M}\right) /(\beta p)$ and magnetic moment $\boldsymbol{M}_{\mathrm{c}}=-\partial F_{\mathrm{c}}[\boldsymbol{M}] / \partial \boldsymbol{H}$ (Dietl et al. 2001c). Another important characteristic of any ferromagnetic system is the magnetic stiffness $A$, which describes the energy penalty associated with a local twisting of the direction of magnetization. Actually, in the experimentally important case of a uniaxial ferromagnet, the energy functional is entirely described by $K_{\mathrm{u}}, A$, and the value of magnetization $M$ according to,

$$
E[\overrightarrow{\vec{n}}(\vec{r})]=E_{\mathrm{o}}+\int \mathrm{d} \vec{r}\left[K_{\mathrm{u}} \sin ^{2} \theta+A(\nabla \overrightarrow{\vec{n}}(\vec{r}))^{2}\right],
$$

where $\hat{\vec{n}}(\vec{r})$ is the unit vector that specifies the local Mn spin orientation, and $\theta$ is its angle with respect to the easy axis. The latter is controlled by biaxial strain in epilayers (Ohno et al. 1996b, Shen et al. 1997) and by confinement in quantum wells (Dietl et al. 1997, Haury et al. 1997). König et al. (2000, 2001) have developed a theory of magnetic stiffness $A$ in III-V ferromagnetic semiconductors. Remarkably, $A$ determines the magnitude and character of thermodynamic fluctuations of magnetization, the width and energy of domain walls as well as the spectrum of spin excitations. In particular, the quantized energies of long-wavelength spin waves are given by

$$
\Omega(\boldsymbol{q})=2 g \mu_{\mathrm{B}}\left(K_{\mathrm{u}}+A \boldsymbol{q}^{2}\right) / M .
$$

It is clear from eq. (17) that $A$ describes how the Ginzburg-Landau functional $F\left[\delta \boldsymbol{M}_{\boldsymbol{q}}\right]$ varies with $\boldsymbol{q}$. Here, $\delta \boldsymbol{M}_{\boldsymbol{q}}$ are the Fourier components of the difference between local and macroscopic magnetization $\boldsymbol{M}(T, \boldsymbol{H})$. In the long-wave limit, in which eq. (17) is valid, the magnitude of $A$ is expected to be primarily determined by the magnetic stiffness of the carrier subsystem, that is by $F_{\mathrm{c}}\left[\delta \boldsymbol{M}_{q}\right]$ and thus by $\tilde{\chi}_{c}^{(i i)}(q)$. Indeed, the distance between the spins is smaller that that between the carriers, and the intrinsic spin-spin interactions are short-range. It is seen, by comparing eqs. (12) and (17), that $\tilde{\chi}_{c}^{(i i)}(\vec{q}, T)$ for the direction $i$ perpendicular to $\boldsymbol{M}(T, \boldsymbol{H})$ is relevant, and its numerical evaluation from eq. (15) for a given $\boldsymbol{M}(T, \boldsymbol{H})$ will provide $A(T, \boldsymbol{H})$. By parametrizing $\tilde{\chi}_{c}^{\perp}(q)=\tilde{\chi}_{c}^{\perp}(q=0)-c_{\perp} q^{2}$ one gets

$$
A=c_{\perp} \beta^{2} M^{2} / 2\left(g \mu_{B}\right)^{2}
$$

As long as the valence band splitting $\Delta=A_{\mathrm{F}} \beta M / g \mu_{\mathrm{B}}$ is much smaller than the Fermi energy one expects the spin susceptibility, and thus $c$ to be independent of $\boldsymbol{M}$, and isotropic for the cubic symmetry $c_{\perp} \approx c_{\|}$. An important observation of König et al. (2001) is that the magnetic stiffness computed within the $6 \times 6$ Luttinger model is almost by a factor of 10 greater than that expected for a simple doubly degenerate band with the heave-hole band-edge mass. This enhancement, which stabilizes strongly the ferromagnetic order, stems presumably from p-like symmetry of the valence band wave functions as well as from interband $q$-dependent polarization.

\subsection{Comparison of theoretical and experimental results}

In this section, the mean-field Zener model discussed above is employed to describe experimental values of Curie temperature, spontaneous magnetization, anisotropy field, and domain stripe width. Standard values of band-structure parameters, elastic constants, and share deformation potentials of GaAs are adopted (Abolfath et al. 2001, Dietl et al. 2001c). The Mn spins are assumed to be in the $\mathrm{d}^{5}$ configuration, so that $S=5 / 2$ and the Mn Landé factor $g=2.0$. For the $\mathrm{p}$-d exchange energy $N_{\mathrm{o}} \beta=-1.2 \mathrm{eV}$ is taken (Okayabashi et al. 1998), which for the cation concentration of GaAs, $N_{\mathrm{o}}=2.21 \times 10^{22} \mathrm{~cm}^{-3}$, corresponds to $J_{\mathrm{pd}} \equiv-\beta=0.054 \mathrm{eVnm}^{3}$. The Fermi liquid parameter $A_{\mathrm{F}}=1.2$ (Jungwirth et al. 1999) enters the enhancement of $T_{\mathrm{C}}$ and of the valence band $\mathrm{p}-\mathrm{d}$ exchange splitting $\Delta=A_{\mathrm{F}} \beta M /\left(\mathrm{g} \mu_{\mathrm{B}}\right)$ at magnetization $M$ of the $\mathrm{Mn}$ spins (Dietl et al. 2001c).

The most interesting property of $\mathrm{Ga}_{1-x} \mathrm{Mn}_{x} \mathrm{As}$ epilayers is the large magnitude of $T_{\mathrm{C}}$, up to $110 \mathrm{~K}$ for the $\mathrm{Mn}$ concentration $x=5.3 \%$ (Ohno et al. 1996b, Matsukura et al. 1998b). Because of this high $T_{\mathrm{C}}$, the spin-dependent extraordinary contribution to the Hall resistance $R_{\mathrm{H}}$ persists up to $300 \mathrm{~K}$, making an accurate determination of the hole density difficult (Oiwa et al. 1997, Van Esch et al. 1997, Matsukura et al. 


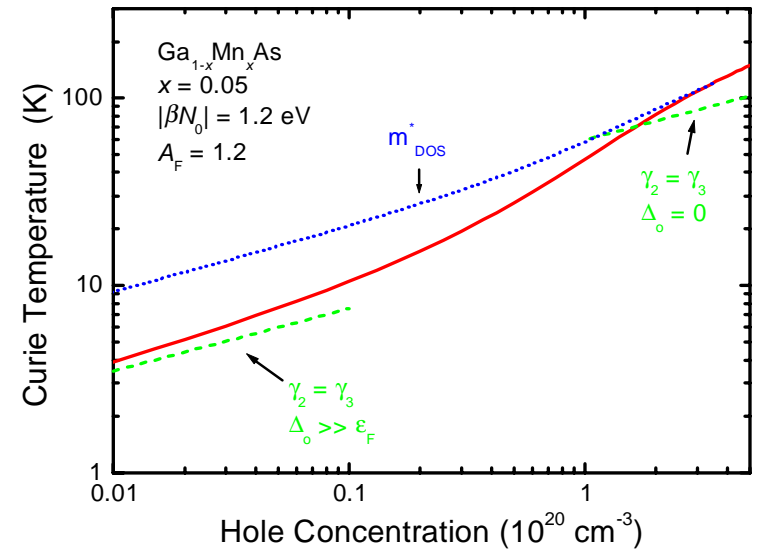

Fig. 25. Curie temperature as a function of the hole concentration for $\mathrm{Ga}_{0.95} \mathrm{Mn}_{0.05} \mathrm{As}$ computed from the $6 \times 6$ Luttinger model (solid line). Straight dashed lines represent results obtained assuming large and small values of the spin-orbit splitting $\Delta_{0}$, respectively. The dotted line is calculated neglecting the effect of the spin-orbit interaction on the hole spin susceptibility (Dietl et al. 2001c).

1998b, Shimizu et al. 1999). However, the recent measurement (Omiya et al. 2000) of $R_{\mathrm{H}}$ up to $27 \mathrm{~T}$ and at $50 \mathrm{mK}$ yielded an unambiguous value of $p=3.5 \times 10^{20} \mathrm{~cm}^{-3}$ for the metallic $\mathrm{Ga}_{0.947} \mathrm{Mn}_{0.053} \mathrm{As}$ sample, in which $T_{\mathrm{C}}=110 \mathrm{~K}$ is observed (Matsukura 1998b). The above value of $p$ is about three times smaller than $x N_{\mathrm{o}}$, confirming the importance of compensation in $\mathrm{Ga}_{1-x} \mathrm{Mn}_{x}$ As.

As shown in Fig. 25, the numerical results lead to $T_{\mathrm{C}}$ $=120 \mathrm{~K}$ for $x=0.05$, and thus, $T_{\mathrm{C}}=128 \mathrm{~K}$ for $x=0.053$ and $p=$ $3.5 \times 10^{20} \mathrm{~cm}^{-3}$. It seems therefore that the mean-field Zener model, with no adjustable parameters, can ecplain the high values of $T_{\mathrm{C}}$ found in $\mathrm{Ga}_{1-x} \mathrm{Mn}_{x}$ As. Furthermore, scaling theory of electronic states near the MIT, discussed in the previous sections, makes it possible to explain the presence of the ferromagnetism on the both sides of the MIT, and a non-critical evolution of $T_{\mathrm{C}}$ across the critical point (Matsukura et al. 1998b). A comparison between theoretical and experimental data in a wider range of $\mathrm{Mn}$ and hole concentrations requires reliable information on the hole density in particular samples, which is not presently available. In appears, however, than in the case of both $\mathrm{Ga}_{1-x} \mathrm{Mn}_{x} \mathrm{As}$ and $\mathrm{In}_{1-x} \mathrm{Mn}_{x} \mathrm{As}$ on the insulator side of the MIT, the experimental values of $T_{\mathrm{C}}$ are systematically higher than those expected from the Zener model.

Turning to the temperature dependence of the spontaneous magnetization in the ferromagnetic phase one should note that the total magnetic moment consists of spin and hole contributions. The hole part was found (Dietl et al. 2001c) to be negative and to attain only a few percent of the total magnetization values, as the hole polarization is incomplete, the hole concentration is smaller than that of the spins, and because of a partial cancellation between the Pauli and Landau terms. If the hole liquid is only partially spin polarized (i.e. $|\Delta|<\left|\varepsilon_{\mathrm{F}}\right|$ ), which is usually the case in $\mathrm{Ga}_{1-x} \mathrm{Mn}_{x} \mathrm{As}, M(T)$ is expected to grow at $T \rightarrow 0$ according to the Brillouin function (Dietl et al. 2001c), in agreement with the experimental results (Matsukura et al. 1998b), shown in Fig. 13. For lower hole concentrations or higher Mn content, $M(T)$ will tend to its saturation value $M_{\mathrm{s}}$ somewhat slower (Dietl et al. 2001c). This, together with a lowering of $M(T)$ by spin wave exitations, may account for the dependence $M(T) / M_{\mathrm{s}}=1-A T^{3 / 2}$, detected experimentally for $T$ $\rightarrow 0$ (Potashnik et al. 2001).

Both hydrostatic and axial strain affect the valence band, and thus alter the magnitude of the density of states and $T_{\mathrm{C}}$. Quantitatively, however, the effect is evaluated to be small (Dietl et al. 2001c). There exists another mechanism by which strain may affect $T_{\mathrm{C}}$. It is presently well known that the upper limit of the achievable carrier concentration is controlled by pinning of the Fermi level by impurity or defect states in virtually all compound semiconductors. Since the energies of such states in respect to bands vary strongly with the bond length, the hole concentration and thus $T_{\mathrm{C}}$ will depend on strain.

Apart from $T_{\mathrm{C}}$ and $M_{\mathrm{s}}$, it is interesting to consider means making it possible to tailor magnetic anisotropy, and thus the direction of the spontaneous magnetization, the coercive force, the switching field, the domain structure. Already early studies of the ferromagnetic phase in $\operatorname{In}_{1-x} \mathrm{Mn}_{x}$ As (Munekata et al. 1993) and $\mathrm{Ga}_{1-x} \mathrm{Mn}_{x}$ As (Ohno et al. 1996b, Shen et al. 1997a) demonstrated the existence of sizable magnetic anisotropy. Magnetic anisotropy is usually associated with the interaction between spin and orbital degrees of freedom of the d-electrons. According to the model advocated here, these electrons are in the $\mathrm{d}^{5}$ configuration. For such a case the orbital momentum $L=0$, so that effects stemming from the spin-orbit coupling are expected to be rather weak. It has, however, been noted that the interaction between the localized spins is mediated by the holes that have a non-zero orbital momentum (Dietl et al. 2000). An important aspect of the Zener model is that it does take into account the anisotropy of the carrier-mediated exchange interaction associated with the spin-orbit coupling in the host material (Dietl et al. 2000, 2001c, Abolfath et al. 2001), an effect difficult to include within the standard approach to the RKKY interaction.

A detail numerical analysis of anisotropy energies has been carried out for a number of experimentally important cases (Dietl et al. 2000, 2001c, Abolfath et al. 2001). In particular, the cubic anisotropy as well as uniaxial anisotropy under biaxial strain have been studied as a function of the hole concentration $p$. The computation indicates that for the parameters of $\mathrm{Ga}_{1-x} \mathrm{Mn}_{x} \mathrm{As}$ films grown along the [001] direction, the spontaneous magnetization $\boldsymbol{M}$ lies in the (001) plane, and the easy axis is directed along the [100] or along the [110] (or equivalent) crystal axis depending on the degree of the occupation the hole subbands as well as on their mixing by the p-d and $\boldsymbol{k} \cdot \boldsymbol{p}$ interactions. As a result, the easy axis fluctuates between [100] and [110] as a function of $\mathrm{p}$, the preferred direction for typical hole concentrations being [110]. The magnitude of the external magnetic field $H_{\mathrm{cu}}$ that aligns $\boldsymbol{M}$ along the hard direction in the (001) plane is evaluated to be up to $0.2 \mathrm{~T}$ (Dietl et al. 2001c). However, the orientation of the easy axis changes rapidly with $p$ and $M$. Therefore disorder, which leads to broadening of hole subbands, will presumably diminish the actual magnitude of magnetic anisotropy. The field $\mu_{\mathrm{o}} H_{\mathrm{cu}}$ determines also the magnitude of the switching field, which could be observed in microstructures containing only a single domain. In macroscopic films, however, smaller values of the coercive field $\mu_{0} H_{c}$ are expected as actually observed: typically $\mu_{0} H_{\mathrm{c}}=4 \mathrm{mT}$ for the magnetic field along the easy axis in the (001) plane in $\mathrm{Ga}_{1-x} \mathrm{Mn}_{x}$ As (Shen et al. 1997a).

It can be expected that strain engineering can efficiently control magnetic properties resulting from the 


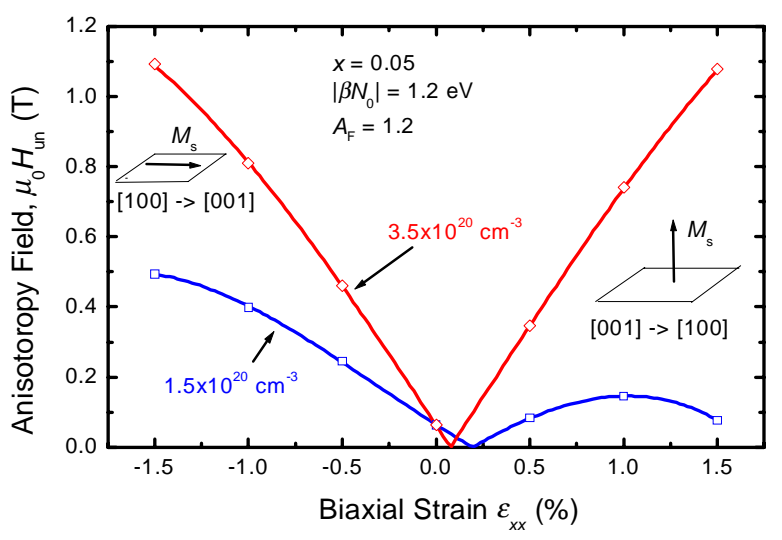

Fig. 26. Computed minimum value of the magnetic field $H_{\mathrm{un}}$ necessary to align the saturation value of magnetization $M_{\mathrm{s}}$ along the hard axis as a function of biaxial strain component $\varepsilon_{\mathrm{xx}}$ for two values of the hole concentrations in $\mathrm{Ga}_{0.95} \mathrm{Mn}_{0.05} \mathrm{As}$. The symbol [100] $\rightarrow$ [001] means that the easy axis is along [100], so that $H_{\mathrm{un}}$ is applied along [001] (Dietl et al. 2001c).

hole-mediated exchange. Indeed, sizable lattice-mismatch driven by biaxial strain is known to exist in semiconductor layers. In some cases, particularly if epitaxy occurs at appropriately low temperatures, such strain can persist even beyond the critical thickness due to relatively high barriers for the formation of misfit dislocations. It has been found that the biaxial strain leads to uniaxial anisotropy, whose magnitude can be much greater than that resulting from either cubic anisotropy or stray fields. As shown in Fig. 26 for the experimentally relevant values of $p$ and $M$, the easy axis is predicted to be oriented along the [001] direction for the tensile strain, whereas it should reside in the (001) plane for the case of unstrained or compressively strained films (Dietl et al. 2000, 2001c, Abolfath et al. 2001). This is corroborated by the experimental study (Ohno et al. 1996b), in which either (In,Ga)As or GaAs substrate was employed to impose tensile or compressive strain in $\mathrm{Ga}_{1-x} \mathrm{Mn}_{x} \mathrm{As}$, respectively. In particular, for the $\mathrm{Ga}_{0.965} \mathrm{Mn}_{0.035} \mathrm{As}$ film on $\mathrm{GaAs}$, for which $\varepsilon_{x x}$ $=-0.24 \%$, the anisotropy field $\mu_{\mathrm{o}} H_{\mathrm{un}}=0.4 \pm 0.1 \mathrm{~T}$ is observed (Ohno et al. 1996b, Shen et al. 1997), in quantitative agreement with the theoretical results of Fig. 26. This field is about two orders of magnitude greater than that evaluated from the extrapolation of ESR data on single-ion anisotropy at low $x$ (Fedorych et al. 2001), a result confirming the dominant contribution of the holes to the magnitude of $H_{\mathrm{un}}$. Though no theoretical computations have been performed for $\operatorname{In}_{1-x} \mathrm{Mn}_{x} \mathrm{As}$, the qualitatively similar effect of biaxial strain is expected, in agreement with results of Munekata et al. (1993), who tailored the easy axis direction by employing $(\mathrm{Al}, \mathrm{Ga}) \mathrm{Sb}$ or $\mathrm{Al}(\mathrm{Sb}, \mathrm{As})$ substrates with various Ga contents or As contents.

Recently, the structure of magnetic domains in $\mathrm{Ga}_{1-x} \mathrm{Mn}_{x} \mathrm{As}$ under tensile strain has been determined by micro-Hall probe imaging (Shono et al. 2000). The regions with magnetization oriented along the [001] and [00 $\overline{1}]$ easy axis form alternating stripes extending in the [110] direction. This indicates, for either Bloch or Néel domain walls, that the in-plane easy axis is rather along [110] than along [100] directions, a conclusion consistent with the theoretical expectation for in-plane (cubic) magnetic anisotropy presented above. As shown in Fig. 27, the experimentally determined stripe width is $W=1.5 \mu \mathrm{m}$ at $5 \mathrm{~K}$ for $0.2 \mu \mathrm{m}$ film of $\mathrm{Ga}_{0.957} \mathrm{Mn}_{0.043}$ As on $\mathrm{Ga}_{0.84} \mathrm{In}_{0.16} \mathrm{As}$, for which a tensile strain of $\varepsilon_{x x}=0.9 \%$ is expected. According to the

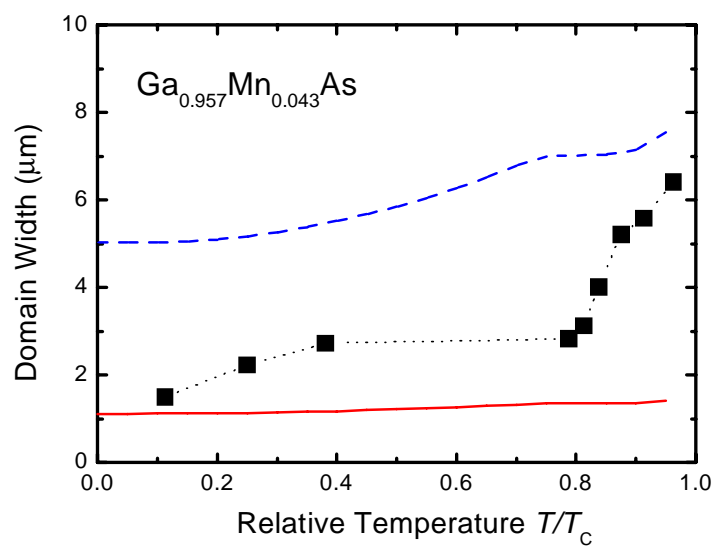

Fig. 27. Temperature dependence of the width of domain stripes as measured by Shono (2000) for the $\mathrm{Ga}_{0.957} \mathrm{Mn}_{0.043} \mathrm{As}$ film with the easy axis along the growth direction (full squares). Computed domain width is shown by the solid line. The dashed line is computed assuming that the parameter $\lambda_{c}$ (eq. (20)) is by a factor of 1.8 greater (Dietl et al. 2001a).

micromagnetic theory, $W$ is determined by the dimensionless parameter $\lambda_{c}$, which is given by the ratio of the domain wall and stray field energies,

$$
\lambda_{\mathrm{c}}=4\left(A K_{\mathrm{u}}\right)^{1 / 2} / \mu_{\mathrm{o}} M^{2} d,
$$

where $d$ is the film thickness.

Figure 27 presents values of $W(T)$ calculated by Dietl et al. (2001a) in comparison to the experimental data of Shono et al. (2000). Furthermore, in order to establish the sensitivity of the theoretical results to the parameter values, the results calculated for a value of $\lambda_{\mathrm{c}}$ that is 1.8 times larger are included as well. The computed value for low temperatures, $W=$ $1.1 \mu \mathrm{m}$, compares favorably with the experimental finding, $W=$ $1.5 \mu \mathrm{m}$. However, the model predicts much weaker temperature dependence of $W$ than observed experimentally, which Dietl et al. (2001a) link to critical fluctuations, disregarded in the mean-field approach.

8.6. Limitations and refinements of the mean-field Zener model

It is obvious that the role of randomness in the distribution of $\mathrm{Mn}$ acceptors and extrinsic defects, which is neglected in the model in question, will grow with approaching the localized regime. According to the two fluid model discussed previously, there is a coexistence of weakly localized carriers and bound magnetic polarons in this regime. In this context particularly interesting are results of Bhatt and Berciu (2001), whose starting point is the impurity band of holes localized by the Mn acceptors. Interestingly, according to their numerical mean-field treatment, positional disorder enhances $T_{\mathrm{C}}$. A further study along the same lines (Kennett et al. 2001) supports the two fluid picture, demonstrating the presence of "strongly" and "weakly" coupled spins. It worth noting that the itinerant carriers may set long-range ferromagnetic correlation between magnetic polarons. Since $T_{\mathrm{C}}$ is proportional to the square of the relevant spin vectors, the weight of the BMP contribution may greatly exceed their relative concentration. The latter, together with the disorder enhancement of $T_{\mathrm{C}}$ mentioned above, may account for higher values of $T_{\mathrm{C}}$ on the insulator side of the MIT than those expected from the Zener model. 
Another important issue requiring further studies is the role of carrier-carrier correlation. It is known that the effect of disorder on carrier-carrier interactions controls the localization and enhances spin susceptibility (Altshuler and Aronov 1985), and thus the tendency towards ferromagnetism. However, spin-disorder scattering may limit the efficiency of this process (Altshuler and Aronov 1985). If this is the case, LSDA (Jungwirth et al. 1999, Lee et al. 2000) can provide a reasonable evaluation of the relevant Fermi-liquid parameter.

There are three other experimentally important situations, for which the mean-field Zener model, as introduced above, ceases to be valid. The first one corresponds to the case when an average time of carrier tunneling between typical Mn pairs $\left(V x_{\text {eff }}^{1 / 3}\right)^{-1}$ becomes significantly longer than the inverse exchange energy $\left|N_{\mathrm{o}} \beta\right|^{-1}$. Here $V$ is the width of the carrier band, and its magnitude, not the Fermi energy as sometimes suggested, constitutes the relevant energy scale. For long tunneling times, the molecular-field and virtual-crystal approximations break down, an effect detected in $\mathrm{Cd}_{1-x} \mathrm{Mn}_{x} \mathrm{~S}$ (Benoit à la Guillaume et al. 1992). A modified double-exchange model will constitute an appropriate description of the carrier-mediated exchange interaction in the strong coupling limit $V<\left|N_{\mathrm{o}} \beta\right|$, where the holes are bound in Zhang-Rice (1988) states, and only occasionally hop between $\mathrm{Mn}$ ions. Here, a strong sensitivity of $T_{\mathrm{C}}$ to the concentration of compensating donors is expected. Dynamic mean-field theory, as developed for III-V magnetic semiconductors by Chattopadhyay et al. (2001), can constitute an appropriate approach in this regime.

Another regime, in which the mean-field Zener model may cease to be valid, is that of large carrier concentrations $n>x_{\text {eff }} N_{0}$. In the limit when the continuous-medium approximation is obeyed, $n<<N_{0}$, the mean-field value of the ordering temperature $T(\boldsymbol{q})$ deduced from the Zener and the RKKY model are identical, independently of microscopic spin arrangement. If, however, $n>x_{\text {eff }} N_{0}$, important changes in the carrier response function occur at the length scale of a mean distance between the localized spins. Accordingly, the description of spin magnetization by the continuous-medium approximation, which constitutes the basis of the Zener model, ceases to be valid. In contrast, the RKKY model is a good starting point in this regime (Ferrand et al. 2001), as it provides the dependence of the interaction energy of particular spin pairs as a function of their distance. This makes it possible to evaluate the system energy for a given distribution of the localized spins. Here, randomness associated with the competition of ferromagnetic and antiferromagnetic interactions can drive the system towards a spin-glass phase (Eggenkamp et al. 1995). In the extreme case, $n$ $>x_{\text {eff }} N_{0}$, the Kondo effect that is dynamic screening of the localized spins by the sea of the carriers may preclude both ferromagnetic and spin-glass magnetic ordering.

Finally, the accuracy of the mean field approximation (MFA) ought to be addressed. It is well known that the MFA results are exact if the range of spin-spin interactions is infinite (Fisher et al. 1972). The decay of the strength of the carrier-mediated exchange interaction with the distance $r$ between two Mn spins is described by the RKKY function. At small $r$, the interaction is ferromagnetic, and then changes sign at $r=1.2 r_{\mathrm{c}}$, where $r_{\mathrm{c}}$ is an average distance between the carriers that mediate spin-spin coupling. This means that the MFA is valid quantitatively at $n \ll x_{\text {eff }} N_{0}$, a conclusion consistent with the estimate of $T_{\mathrm{C}}$ taking the spin wave excitations into account (König et al. 2000). Actually, however, the range of validity of the MFA is significantly larger than that initially found (König et al. 2000), as the magnitudes of spin stiffness evaluated within the $6 \times 6$ Luttinger model are much greater (König et al. 2001) than those obtained for a simple parabolic band (König et al. 2000).

Recently, Monte-Carlo studies of carrier-mediated ferromagnetism in semiconductors have been initiated (Sakai et al. 2001, Sakai and Suzuki 2001, Bosselli et al. 2000, Schliemann et al. 2001a, 2001b). Such an approach has a potential to test the accuracy of the approximations mentioned above and to determine the actual spin configuration corresponding to the ground state. Preliminary results appear to confirm the validity of the MFA (Sakai et al. 2001, Bosselli et al. 2000), and indicate a possibility of the existence of non-collinear magnetic structures in low-dimensional systems (Bosselli et al. 2000). More recent comprehensive simulations of Schliemann et al. (2001b) carried out within the hybrid Monte Carlo scheme, identify the parameter space, in which the mean-field Zener model may break down. It would be interesting to check separately the regions of validity of particular approximations involved: MFA, virtual crystal approximation (VCA), and molecular-field approximation, as well as to elucidate the role of electrostatic disorder.

\section{Heterostructures}

New physics such as the fractional quantum Hall effect has emerged from non-magnetic semiconductor heterostructures. These systems have also been a test bench for a number of new device concepts, among which are quantum well lasers and high electron mobility transistors. Ferromagnetic III-Vs can add a new dimension to the III-V heterostructure systems because they can introduce magnetic cooperative phenomena that were not present in the conventional III-V materials.

\subsection{Basic properties of heterostructures}

\subsubsection{Structural properties of multilayer structures}

Figure 28 shows a typical double-crystal x-ray diffraction pattern of the (004) reflection of a GaAs/(Ga,Mn)As superlattice grown on a GaAs (001) substrate, taken by employing $\mathrm{CuK \alpha} \alpha_{1}$ radiation (Shen et al. 1997d). The sample consists of 20 periods of nominally 11.4-nm thick GaAs and 12.1-nm thick (Ga,Mn)As with $x=0.054$. Satellite peaks witnessing the periodicity of the structure are clearly visible. Theoretical simulations of the rocking curve were carried out by adding background noise of $10 \mathrm{cps}$. The values of the elastic constants and the Debye-Waller factor determined for GaAs were adopted for zinc-blende MnAs. The best fit shown in Fig. 28 reveals that $x=0.056$ as well as that the thicknesses of the $\mathrm{GaAs}$ and $(\mathrm{Ga}, \mathrm{Mn}) \mathrm{As}$ layers are $11.4 \mathrm{~nm}$ and $11.70 \mathrm{~nm}$, respectively, in good agreement with the nominal values. The fact that almost the same line width is obtained for the experimental and the simulated satellite peaks suggests a high quality of the films and the interfaces. The high perfection of the 


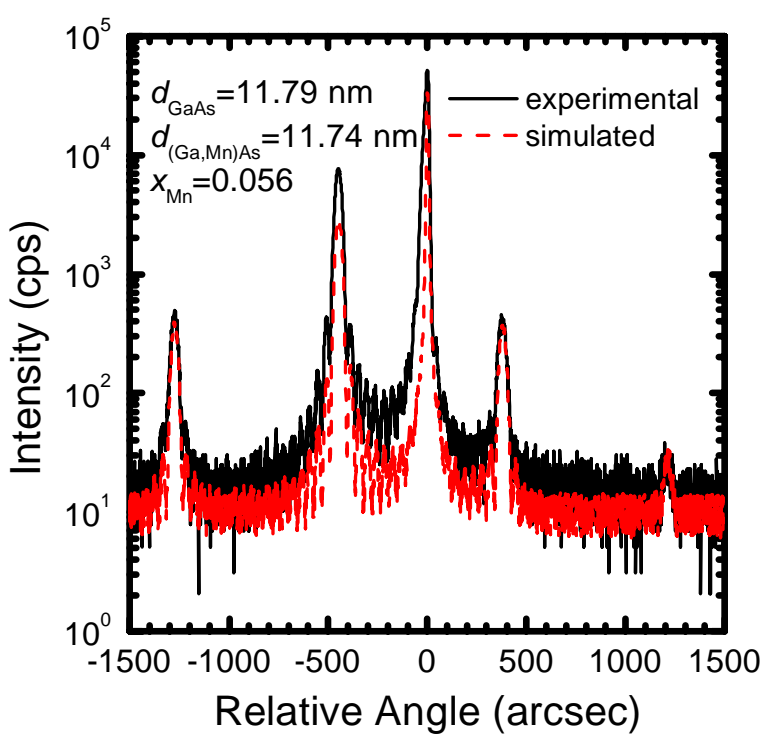

Fig. 28. X-ray diffraction rocking curve of (Ga,Mn)As/GaAs superlattices with 20 periods (solid line). The nominal thicknesses of the GaAs and (Ga,Mn)As layers and the Mn composition $x$ are $11.4 \mathrm{~nm}, 12.1 \mathrm{~nm}$, and 0.054, respectively. The dashed line shows the simulated rocking curve. The fit to the experimental curve is obtained with GaAs and $(\mathrm{Ga}, \mathrm{Mn})$ As thicknesses and $x$ of $11.14 \mathrm{~nm}, 11.79 \mathrm{~nm}$, and 0.056, respectively (Shen et al. 1997d).

structure is also confirmed by the observation of thickness fringes due to perfect crystal diffraction from thin films.

\subsubsection{Magnetic properties of supelattices, thin films, and quantum wells}

Transport measurements on multilayer structures demonstrate that the ferromagnetism can be retained down to a (Ga,Mn)As layer thickness of $5 \mathrm{~nm}$, below which the structure becomes paramagnetic. On the other hand, there was a report on the persistence of ferromagnetic properties in short-period superlattices consisting of $m$ (Ga,Mn)As and $n$ GaAs monolayers, with $8 \leq m \leq 12$ and $4 \leq n \leq 8$ (Sadowski et al. 2001e). The reason for these diverging conclusions is not clearly understood; it may be related to the Mn segregation at the initial stage of growth which leads to a depletion of $\mathrm{Mn}$ and/or the distribution of the spin-polarizaed carrier (Louriero da Silva et al. 2001, Vurgaftman and Meyer 2001). Multiple (Ga,Mn)As quantum wells (QWs) were also fabricated and studied by means of magnetization, magneto-optical and magneto-transport phenomena (Hayashi et al. 1997b, 1998). No ferromagnetic order in $(\mathrm{Ga}, \mathrm{Mn}) \mathrm{As}$ QWs with thickness less than $5 \mathrm{~nm}$ is observed. MCD results show clear evidence of the quantum confinement and the formation of subband.

Figure 29 shows the thickness dependence of magnetization in $(\mathrm{Ga}, \mathrm{Mn})$ As films, as determined by the Hall effect (note that $R_{\text {Hall }} / R_{\text {sheet }} \propto M$ ) (Matsukura et al. 1998a). The inset presents the values of $T_{\mathrm{C}}$ in these films. Apart from an increase in anisotropy and a gradual growth of $T_{\mathrm{C}}$, no significant changes are observed when the film thickness is reduced from 1 $\mu \mathrm{m}$ to $10 \mathrm{~nm}$. Below $5 \mathrm{~nm}$, however, the (Ga,Mn)As films become insulating. At the same time, $T_{\mathrm{C}}$ drops significantly, often below the lowest studied temperature of $2 \mathrm{~K}$. Similarly, the ferromagnetism disappears in $(\mathrm{Ga}, \mathrm{Mn}) \mathrm{As} \mathrm{QWs}$, if their

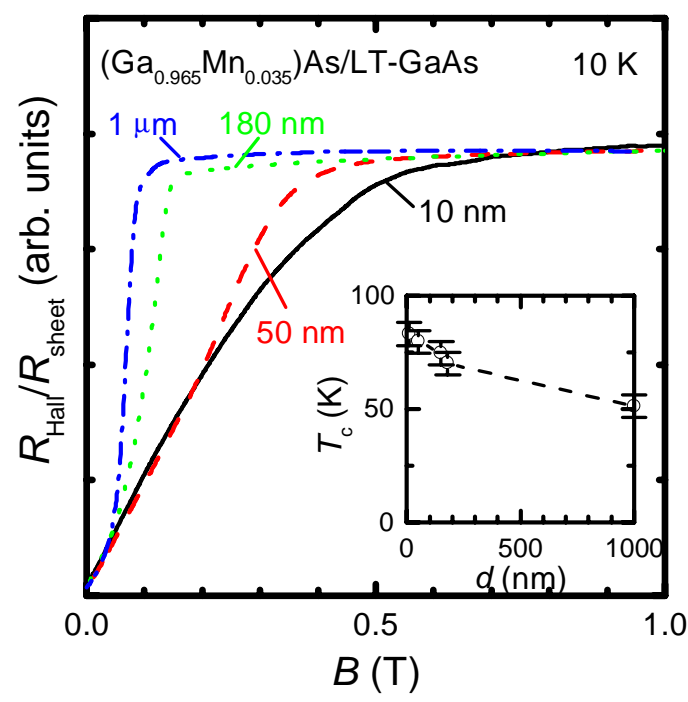

Fig. 29. Thickness dependence of the ratio of Hall resistance and sheet resistance $R_{\text {Hall }} / R_{\text {sheet }}$, which is proportional to magnetization perpendicular to the film plane, as a function of the magnetic field at $10 \mathrm{~K}$. The inset shows the thickness dependence of $T_{\mathrm{C}}$ (Matsukura et al. 1998a).

width is below $5 \mathrm{~nm}$ (Hayashi et al. 1997b). No systematic differences in the behavior of superlattices, thin films or quantum wells have been detected. In contrast, the ferromagnetism was observed in GaAs samples to which a submonolayer of MnAs was inserted (Kawakami et al. 2000). Further studies are apparently necessary to understand the thickness dependence of magnetic properties in these materials.

\subsubsection{Band offset between $(\mathrm{Ga}, \mathrm{Mn}) \mathrm{As}$ and $\mathrm{GaAs}$}

A heterojunction is primarily characterized by the band alignment. This most fundamental property of any

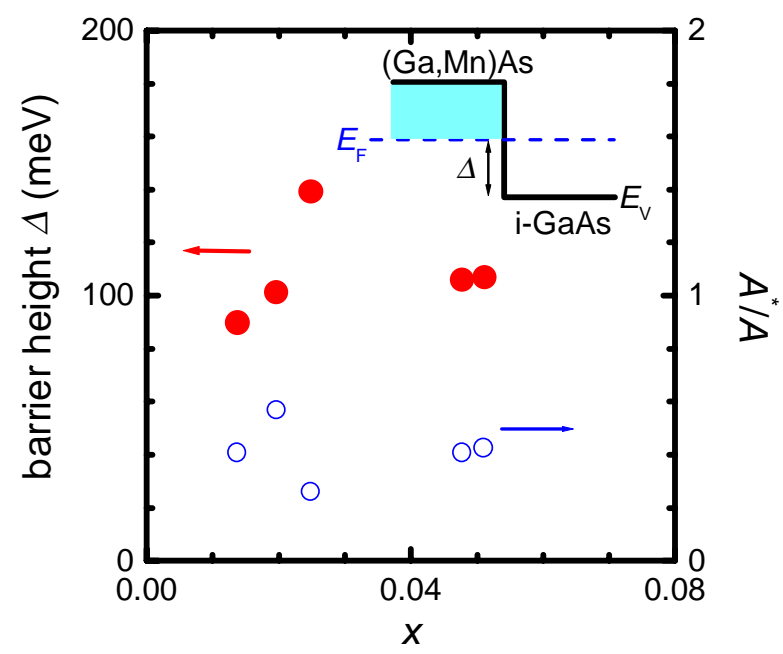

Fig. 30. Barrier height $\Delta$ measured by current-voltage $(I-V)$ characteristics of $(\mathrm{Ga}, \mathrm{Mn}) \mathrm{As} / \mathrm{GaAs}$ diodes. $\Delta$ shown by closed circles is the barrier height between the Fermi energy of $(\mathrm{Ga}, \mathrm{Mn}) \mathrm{As}$ and the valence band top of GaAs as shown in the inset. Open circles depict the effective Richardson constants. (Ohno et al. 2001). 
heterojunction is not well established in the case of Mn-doped III-V's. The difficulty in determining the band offset arises from a number of reasons, such as a high doping level in Mn-doped III-V's and a relatively small values of $\mathrm{Mn}$ concentrations, which results in a tiny magnitude of the band offset. The close examination of current-voltage $(I-V)$ characteristics of $\mathrm{p}-\mathrm{i}-\mathrm{n}$ and p-p diodes made of (Ga,Mn)As and GaAs shows that thermoionic emission at te mperature higher than $T_{\mathrm{C}}$ is the dominant mechanism of the current transport (Ohno, Y. et al. 2000, Ohno et al. 2001, Arata et al. 2001). By analyzing the temperature dependence of the $I-V$ characteristics, one can deduce the barrier height between $(\mathrm{Ga}, \mathrm{Mn}) \mathrm{As}$ and GaAs measured from the Fermi level of (Ga,Mn)As. Results of such an analysis are depicted in Figure 30 (Ohno et al. 2001), which presents the measured barrier height $\Delta$ as a function of $x$, together with the associated effective Richardson constant $\left(A^{*} / A\right)$. Inset shows the band structure of the measured samples. The findings demonstrate that the holes flowing from the (Ga,Mn)As side have to overcome a barrier of about $100 \mathrm{meV}$, the value being virtually independent of $x$. However, the interpretation of this number and the determination of the bare offset $\Delta E_{\mathrm{v}}$ between $(\mathrm{Ga}, \mathrm{Mn}) \mathrm{As}$ and GaAs valence band edges is by no means straightforward. In particular, the Fermi energy of (Ga,Mn)As (usually of the order of $100 \mathrm{meV}$ ) has to be taken into account, which will result in $\Delta E_{\mathrm{v}} \approx 200 \mathrm{meV}$. At the same time, however, band gap renormalization caused by the hole-hole interactions, the hole coupling to the system of ionized impurities, and the impurity band formation will shift the $(\mathrm{Ga}, \mathrm{Mn}) \mathrm{As}$ valence band edge towards higher energies, reducing $\Delta E_{\mathrm{v}}$.

9.2. Spin-dependent scattering, interlayer coupling, and tunnel magnetoresistance in trilayer structures

Ferromagnet/nonmagnet/ferromagnet trilayer structures constitute the most fundamental building block of

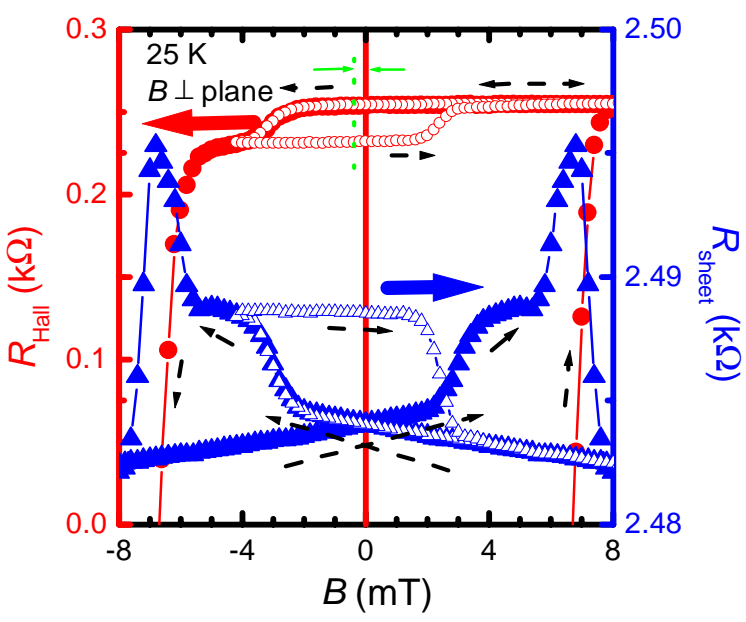

Fig. 31. Hall resistance $R_{\text {Hall }}$ (circles) and sheet resistance $R_{\text {sheet }}$ (triangles) versus magnetic field $B$ at $25 \mathrm{~K}$ for a $\left(\mathrm{Ga}_{0.95} \mathrm{Mn}_{0.05}\right) \mathrm{As} /\left(\mathrm{Al}_{0.14} \mathrm{Ga}_{0.86}\right) \mathrm{As} /\left(\mathrm{Ga}_{0.97} \mathrm{Mn}_{0.03}\right) \mathrm{As} \quad$ trilayer structure. Closed and open symbols show the major and minor loops, respectively. Dashed arrows indicate sweep directions of the magnetic field. The minor loop of $R_{\text {Hall }}$ is skewed by the presence of a ferromagnetic coupling between the two (Ga,Mn)As layers (Chiba et al. 2000). modern magnetic sensors and storage elements, and are useful for the examination of various magnetotransport processes. Especially important is spin-dependent scattering in such structures, as this is the basic process that gives rise to the effect of giant magnetoresistance (GMR). Equally important is the effect of tunneling magnetoresistance (TMR) in structures containing a thin insulator as the nonmagnetic layer. In order to investigate the nature of spin-dependent transport in systems made of semiconductors only, various (Ga,Mn)As/(Al,Ga)As/(Ga,Mn)As structures have been prepared and studied (Akiba et al. 1998, 2000a, Hayashi et al. 1999, Chiba et al. 2000, Higo et al. 2001a, 2001b).

Figure 31 presents the field dependence of the Hall resistance and the magnetoresistance at $25 \mathrm{~K}$ for a Hall bar pattern of a trilayer structure (Chiba et al. 2000). The device consists of two $30 \mathrm{~nm} \mathrm{Ga}_{1-x} \mathrm{Mn}_{x}$ As layers separated by a $2.8 \mathrm{~nm}$ nonmagnetic $\mathrm{Al}_{0.14} \mathrm{Ga}_{0.86} \mathrm{As}$ layer, acting as a barrier for holes, whose height depends on the $\mathrm{Al}$ concentration. The layers are grown onto a $50 \mathrm{~nm} \mathrm{Al}_{0.30} \mathrm{Ga}_{0.70} \mathrm{As}$ buffer and a $1 \mu \mathrm{m}$ relaxed $\mathrm{In}_{0.15} \mathrm{Ga}_{0.85} \mathrm{As}$ film. The Mn content $x=0.05$ and 0.03 of the two $(\mathrm{Ga}, \mathrm{Mn})$ As layers results in a difference of their coercive forces. The $\left(\operatorname{In}_{0.15} \mathrm{Ga}_{0.85}\right)$ As film introduces a tensile strain, which makes the magnetic easy axis to be perpendicular to the structure plane. With this easy axis direction, the Hall effect can be used to monitor magnetization.

Parallel transport in trilayer structures is characterized by the MR ratio $\left(R_{\text {sheet }}-R_{0}\right) / R_{0}$, where $R_{0}$ is the layer resistance in the absence of the external magnetic field, $R_{\text {sheet }}(B=0)$, and for parallel magnetizations $M$ of the two (Ga,Mn)As layers. A plateau seen in the Hall resistance data collected in Fig. 31, indicates that magnetizations of the two (Ga,Mn)As layers are anti-parallel (note that $R_{\text {Hall }}$ is

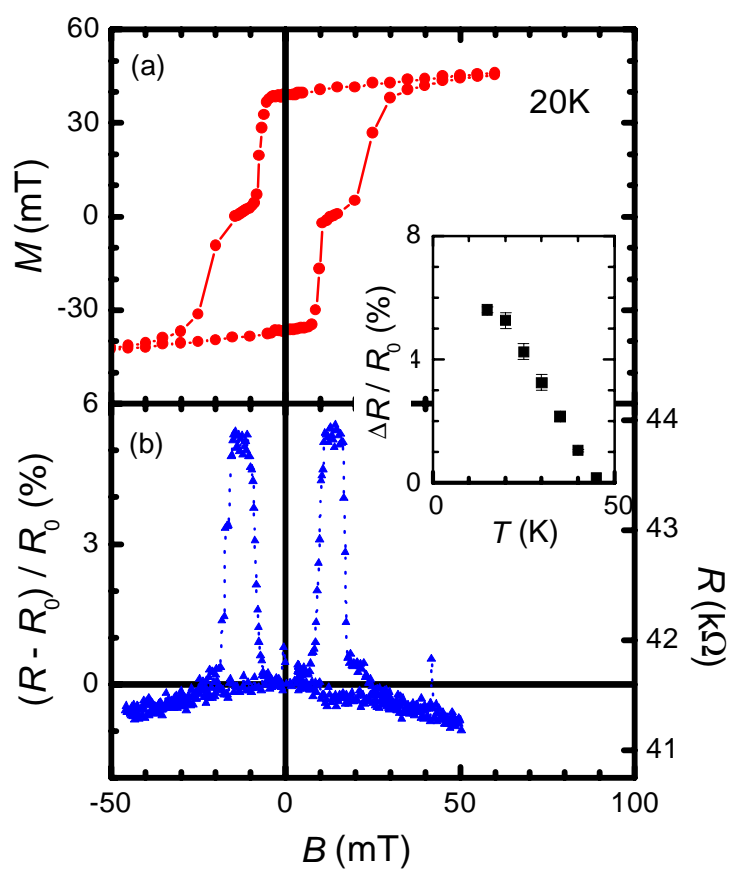

Fig. 32. (a) Magnetization $M$ and (b) tunneling magnetoresistance of $30 \mathrm{~nm}\left(\mathrm{Ga}_{0.95} \mathrm{Mn}_{0.05}\right) \mathrm{As} / 3 \mathrm{~nm}$ AlAs/ 30 $\mathrm{nm}\left(\mathrm{Ga}_{0.97} \mathrm{Mn}_{0.03}\right)$ As tunnel junction at $20 \mathrm{~K}$. Inset shows the temperature dependence of tunneling magnetoresitance ratio (Chiba et al. 2000). 

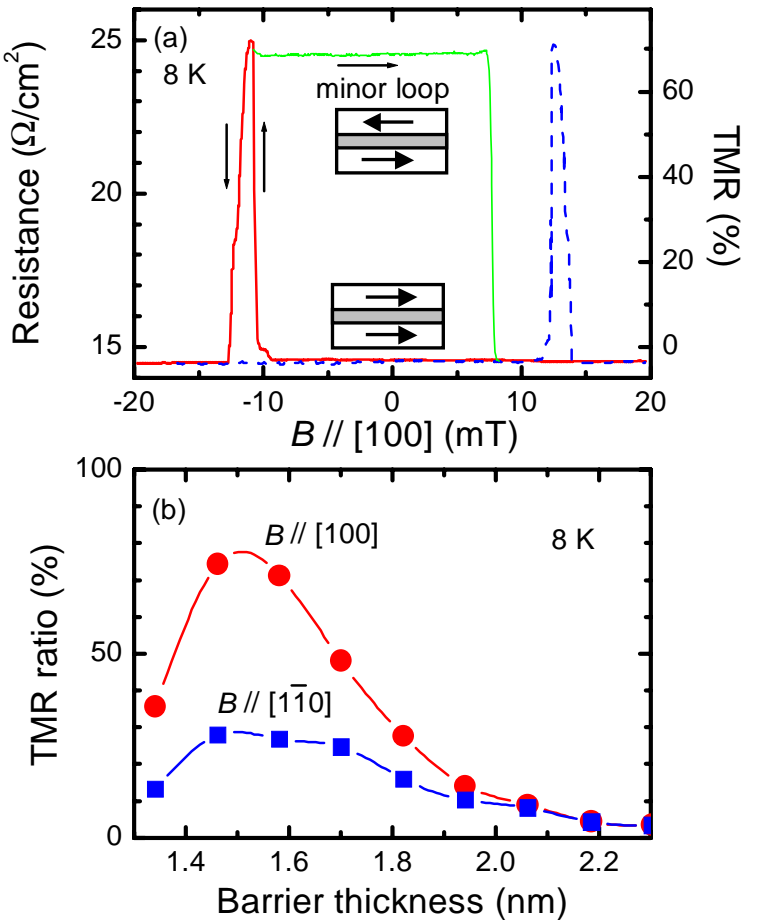

Fig. 33. (a) TMR curves at $8 \mathrm{~K}$ of a $\mathrm{Ga}_{1-x} \mathrm{Mn}_{x}$ As $(x=4.0 \%, 50$ $\mathrm{nm}) / \mathrm{AlAs}(1.6 \mathrm{~nm}) / \mathrm{Ga}_{1-x} \mathrm{Mn}_{x} \mathrm{As}(x=3.3 \%, 50 \mathrm{~nm})$ tunnel junction $200 \mu \mathrm{m}$ in diameter. Bold solid and dashed curves were obtained by sweeping the magnetic field from positive to negative and negative to positive, respectively. A minor loop is shown by a thin solid curve. The magnetic field was applied along the [100] axis in the plane. (b) Barrier thickness dependence of TMR values with the magnetic field applied along the [100] and $[1 \overline{1} 0]$ axes at $8 \mathrm{~K}$ (Tanaka and Higo 2001).

proportional to a weighted average of $M$ of the two (Ga,Mn)As layers). A clear increase of the MR ratio is observed in the plateau region between 3 and $6 \mathrm{mT}$. This demonstrates the presence of spin-dependent scattering in the trilayer structures made of semiconductors only. The MR ratio decreases with the increase of $\mathrm{Al}$ composition in the barrier, which reduces the number of holes traveling across it.

In the minor loop measurements, when the direction of magnetization of one of the two layers is fixed, the hysteresis loops of $R_{\text {Hall }}$ and the MR ratio show a good correspondence (open symbols in Fig. 31). This confirms that the observed MR effect is indeed due to spin-dependent scattering. The minor loop is shifted away from $B=0$, which indicates that there is a ferromagnetic interlayer coupling between the two (Ga,Mn)As layers. The magnitude of this coupling estimated from this shift is quite small $\left(<1 \mu \mathrm{J} / \mathrm{m}^{2}\right)$. The coupling is always ferromagnetic, although theory predicts an antiferromagnetic interaction under certain sets of parameters (Jungwirth et al. 1999). The magnitude of the coupling increases with the lowering of the $\mathrm{Al}$ composition in the $(\mathrm{Al}, \mathrm{Ga}) \mathrm{As}$ barrier. This suggests that the interlayer coupling is mediated by holes. Elastic neutron scattering and polarized neutron reflectivity measurements on short-period (Ga,Mn)As/GaAs superlattices show also the presence of a ferromagnetic interlayer coupling (Szuszkiewicz et al. 2001, Kępa et al. 2001). An interlayer coupling between two (In,Mn)As layers separated by InAs has also been observed (Yanagi et al. 2001).

Vertical transport measurements of trilayer
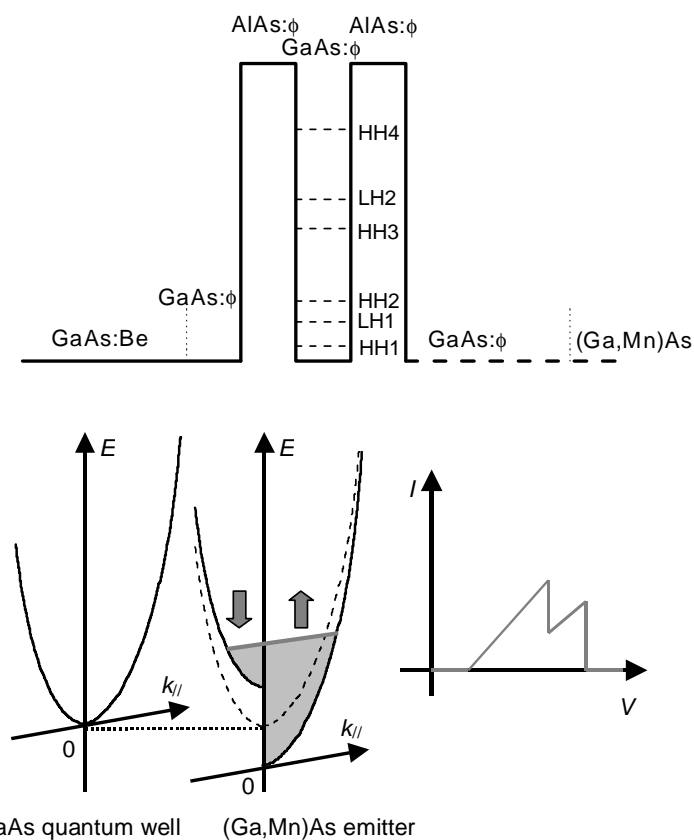

Fig. 34. Schematic valence band diagram of resonant tunneling diode structures, simplified diagram of energy versus wave vector parallel to the interface, and resulting $I-V$ curve by spin-splitting of the valence band of (Ga,Mn)As emitter.

structures reveal the presence of TMR. Figure 32 (a) shows findings obtained at $20 \mathrm{~K}$ for a device containing a $3 \mathrm{~nm}$ AlAs barrier (Chiba et al. 2000). The structure was grown on a (In,Ga)As film to fix the magnetization direction perpendicular to the plane. For TMR measurements, the electrodes were formed on the top and bottom $(\mathrm{Ga}, \mathrm{Mn}) \mathrm{As}$ layers. The temperature dependence of the MR ratio is shown in the inset to Fig. 32. The difference in the coercive forces produces a plateau visible in Fig. 32 (a), in the region, where magnetizations of the two ferromagnetic layers are antiparallel. A resistance increase is observed between $8 \mathrm{mT}$ and $16 \mathrm{mT}$, in the field region corresponding to an antiparallel configuration of magnetizations. The MR ratio is about $5.5 \%$ at $20 \mathrm{~K}$. This is TMR, because the barrier produced by AlAs is high $(0.55 \mathrm{eV})$, so that the hole transport across the AlAs layer proceeds by tunneling. The TMR ratio decreases with temperature most probably due to a corresponding drop of spontaneous magnetization in (Ga,Mn)As layers. A TMR ratio over $70 \%$ was observed in a (Ga,Mn)As/AlAs/(Ga,Mn)As structure with an AlAs thickness of 3 monolayers $(1 \mathrm{~nm})$ as shown in Fig. 33. Such a high value indicates that spin polarization of carriers in $(\mathrm{Ga}, \mathrm{Mn})$ As is quite high (Higo et al. 2001a, 2001b, Tanaka and Higo 2001). A theoretical calculation of TMR with ferromagnetic DMS electrodes shows how the TMR ratio depends on the Mn and hole concentrations (Lyu and Moon 2001).

\subsection{Resonant tunneling diodes (RTDs)}

Spontaneous magnetization in ferromagnetic semiconductors gives rise to spin splitting of the conduction and valence bands due to the presence of exchange interaction. This spin splitting can be observed in current-voltage (I-V) characteristics of resonant-tunneling diodes (RTDs) having a ferromagnetic emitter as shown in Fig. 34. Nonmagnetic double 


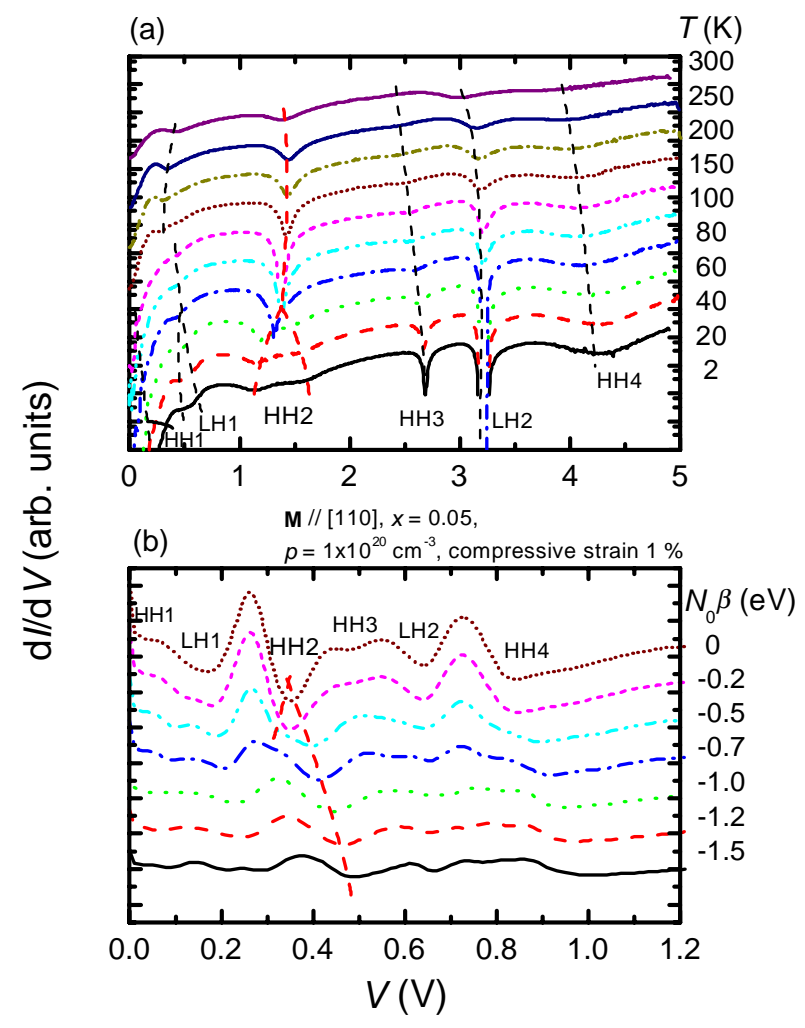

Fig. 35. Temperature dependence of the differential conductance $\mathrm{d} I / \mathrm{d} V$ versus bias voltage $V$ of a resonant tunneling diode with a $(\mathrm{Ga}, \mathrm{Mn}) \mathrm{As}$ emitter. No magnetic field is applied (Ohno et al. 1998). (b) Calculated resonant tunneling spectra as a function of the exchange energy $N_{0} \beta$ (Akiba et al. 2000b).

barrier AlAs/GaAs/AlAs RTDs with a (Ga,Mn)As emitter reveal spontaneous splitting of resonant peaks below $T_{\mathrm{C}}$ of $(\mathrm{Ga}, \mathrm{Mn}) \mathrm{As}$, even in the absence of an external magnetic field (Ohno et al. 1998, Akiba et al. 1998). Figure 35 (a) shows the temperature dependence of $\mathrm{d} I / \mathrm{d} V$ versus $V$ of an RTD having a (Ga,Mn)As emitter. Clear spontaneous splitting of resonant peaks labeled $\mathrm{HH} 2$ and LH1 is observed below $T_{\mathrm{C}}$ of $60 \mathrm{~K}$. The structure in question was grown onto a $\mathrm{p}^{+} \mathrm{GaAs}$ substrate and consists of several layers (from top): $150 \mathrm{~nm}\left(\mathrm{Ga}_{0.965} \mathrm{Mn}_{0.035}\right) \mathrm{As} / 15 \mathrm{~nm}$ undoped GaAs spacer/ $5 \mathrm{~nm}$ undoped AlAs barrier/ $5 \mathrm{~nm}$ undoped GaAs QW/ $5 \mathrm{~nm}$ undoped AlAs barrier/ $5 \mathrm{~nm}$ undoped GaAs spacer/ $150 \mathrm{~nm}$ Be doped GaAs $\left(p=5 \times 10^{17} \mathrm{~cm}^{-3}\right) / 150$ $\mathrm{nm}$ Be doped GaAs $\left(p=5 \times 10^{18} \mathrm{~cm}^{-3}\right)$. Each label in Fig. 35 indicates the resonant state in the GaAs well, where in total six states occur.

Akiba et al. (2000b) performed a calculation of the corresponding $\mathrm{d} I / \mathrm{d} V-V$ characteristics, taking into account a strong $k$-dependent mixing and the presence of the exchange interaction. Previous theoretical approaches to p-type RTD's (Chao and Chuang 1991, Rodrigues Bittencourt et al. 1998) were supplemented by incorporating the p-d exchange interaction into the $6 \times 6$ Luttinger-Kohn Hamiltonian. The resonant states in the GaAs well were calculated by using the $4 \times 4$ Hamiltonian. The current density $J_{z}$ for a given hole concentration and voltage was then calculated as,

$J_{z}=\frac{e}{(2 \pi)^{3}} \sum_{i, j, l=1}^{4} \iiint_{E \leq E_{F}} d^{3} k \cdot\left(\frac{\partial E}{\partial k_{z}}\right) \cdot T^{*} T_{i j} \cdot[f(E)-f(E-e V)] \cdot c_{i l}$
Here, $T^{*} T_{i j}$ is the transmission coefficient from the heavy or light hole state in the emitter to the heavy and light hole states in the collector, $f(E)$ the Fermi-Dirac distribution function, $c_{i l}$ is the probability amplitude of the $j$ th hole state in the $l$ th band. Figure 35 (b) shows the calculated $\mathrm{d} I / \mathrm{d} V-V$ characteristics with $\left|N_{0} \beta\right|$ as a parameter, which corresponds to various values of magnetization in the present case. A $1 \mathrm{~nm}$ AlAs barrier is assumed to avoid numerical instability. A $5 \%$ compressive strain is included in the $(\mathrm{Ga}, \mathrm{Mn}) \mathrm{As}(x=0.05)$ layer containing $1 \times 10^{20}$ holes per $\mathrm{cm}^{3}$. The magnetization is assumed to be in plane along the [110] direction. As can be seen in Fig. 35 (b), the $\mathrm{HH} 2$ resonant peaks shows a clear splitting as $N_{0} \beta$ (magnetization) increases, whereas other peaks (except probably LH1) do not show such pronounced splitting, the conclusions being in accord with the findings. The splitting of the $\mathrm{HH} 2$ peak is so pronounced because the dispersion of the $\mathrm{HH} 2$ resonance is similar to that of the dominant valence band state in the emitter. Other resonant states either show a very different dispersion and/or lie high in energy, where transmission maxima become broad. Note that no clear cut-off of the current is observed neither in the experiment nor in theory because of a rather high hole concentration. The present results indicate that because of a complex interplay between the $k \cdot p$ and exchange interactions, it is necessary to go through a rather elaborate calculation to understand the origin of the peaks and their splitting. Similar calculations were performed for different structures with a DMS QW and adopting Keldysh formalism (Petukhov et al. 2000, Inoue et al. 2000, Nonoyama and Inoue 2001, Kuivalainen and Hovinen 2002).

9.4. Spin-injection in ferromagnetic semiconductor heterostructures

Spontaneous magnetization in ferromagnetic semiconductors introduces an imbalance in the spin population of the carries. Thus, these materials can be used as a source of spin polarized carriers, which can be injected into non-magnetic structures. In spite of the common belief that very fast hole spin relaxation precludes the effect, hole spin injection from (Ga,Mn)As into GaAs was demonstrated (Ohno, Y. et al. 1999). Figure 36 (a) shows a schematic diagram of a light emitting diode employed to demonstrate the spin injection. Partly spin polarized holes are injected from a p-type (Ga,Mn)As layer through an intrinsic GaAs spacer into an (In, Ga)As QW, where they recombine with unpolarized electrons injected from nonmagnetic n-type GaAs substrate. The spin polarization of the recombining holes and hence spin injection is demonstrated by the observation of electroluminescence (EL) polarization. Figure 36 (b) shows the relative polarization change $\Delta P$ as a function of the magnetic field at various temperatures. Two polarization states are clearly observed at $B=0$ at low temperatures. A series of experiments was done to exclude effects of both fringing fields and magnetic circular dichroism from the adjacent (Ga,Mn)As. Because the easy direction is in-plane for $(\mathrm{Ga}, \mathrm{Mn}) \mathrm{As}$ grown on GaAs, EL was collected along the direction parallel to the QW plane. Since the selection rule at the very bottom of the band in QW does not allow emission of circularly polarized light in this direction, the effect of band filling (i.e. nonzero $k$ ) needs to be taken into account to 
(a)

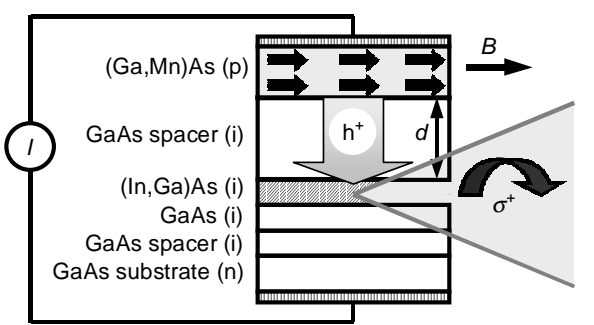

(b)

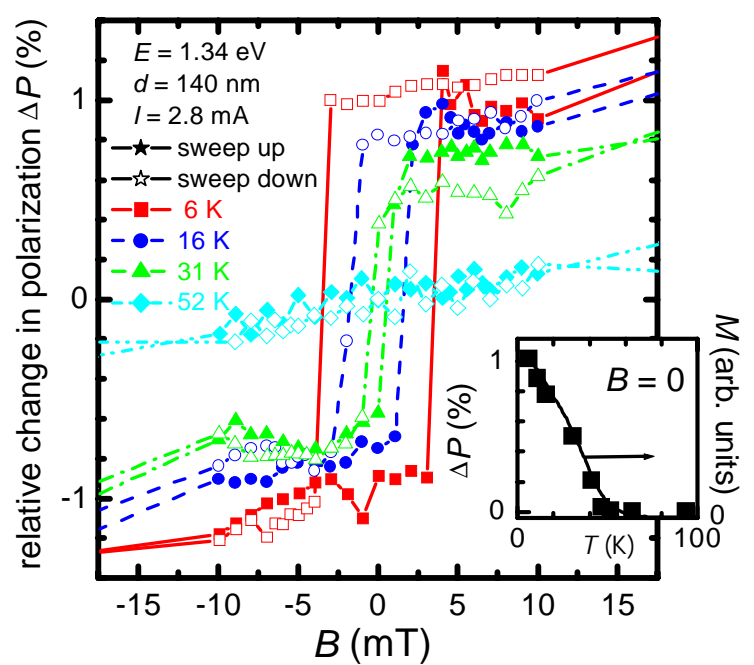

Fig. 36. (a) The structure of a light emitting diode for detection of electrical spin-injection. (b) Relative change in light polarization $\Delta P$ as a function of the magnetic field at four different temperatures. Inset compares the remanent magnetization as determined by SQUID magnetomery with the remanent $\Delta P$ (Ohno, Y. et al. 1999).

fully understand the results.

Injection of electron spins is preferable from the application point of view as electrons usually exhibit longer spin lifetime. Spin-injection experiments using n-type II-VI DMS in the magnetic field were performed by Fiederling et al. (1999) and Jonker et al. (2000). An appealing scheme for electrical electron spin injection from a ferromagnetic material is the use of a broken gap heterojunction system such as $\mathrm{InAs} / \mathrm{GaSb}$, in which the valence band edge of $\mathrm{GaSb}$ is energetically higher than the conduction band edge of InAs. The successful growth of ferromagnetic $(\mathrm{Ga}, \mathrm{Mn}) \mathrm{Sb}$ should make it possible to inject spin polarized electrons into nonmagnetic InAs (Abe et al. 2000). Another way is to use an Esaki tunnel diode, a method successfully developed by Koda et al. (2001) and by Johnston-Halperin et al. (2002), who employed p-(Ga,Mn)As/n-GaAs diodes.

By solving the drift-diffusion equation for a GaAs-based pn junction, it has been shown theoretically that the spin-polarization in the $\mathrm{p}$ region can be transfered into the $n$ region with high efficiency through the depletion layer (Žutic et al. 2001). In this structure the spin polarization in the $\mathrm{n}$ region can be controlled by the external applied electronic bias; i.e., an increase of forward bias results in are decrease of the spin polarization.

\subsection{Photo-induced ferromagnetism in ( $\mathrm{In}, \mathrm{Mn}) \mathrm{As} / \mathrm{GaSb}$}

The relationship between $T_{\mathrm{C}}$ and the hole
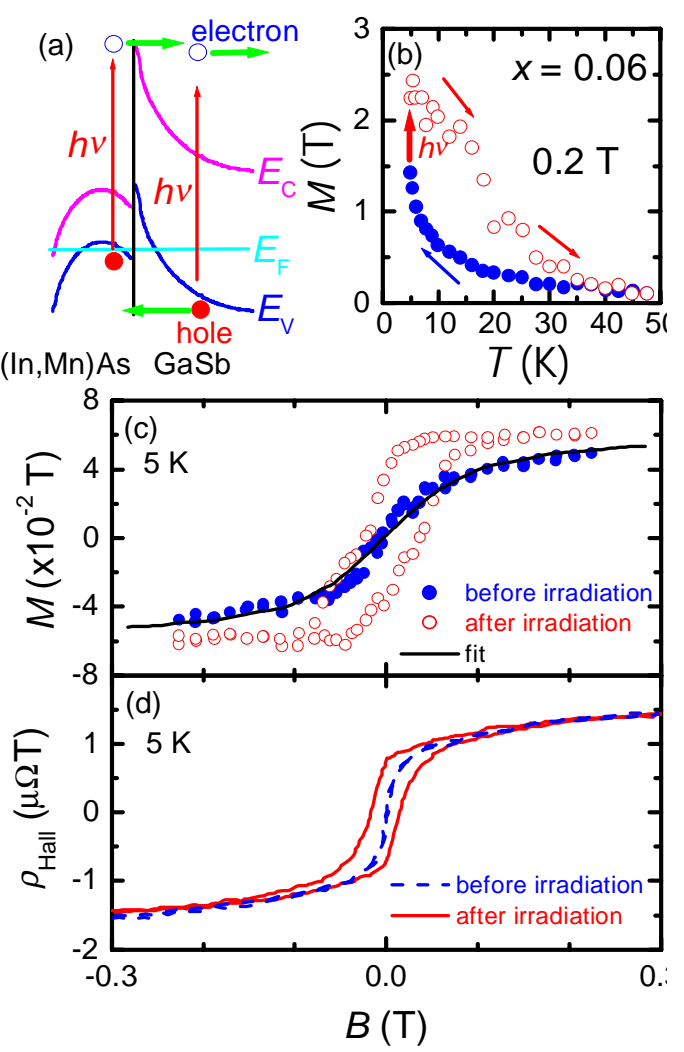

Fig. 37. Band edge profile of a (In,Mn) $\mathrm{As} / \mathrm{GaSb}$ heterostructure. $E_{\mathrm{C}}, E_{\mathrm{V}}$, and $E_{\mathrm{F}}$ denote band edges of conduction band, valence band, and Fermi level, respectively. (b) Temperature dependence of magnetization observed during cooldown in the dark (open circles) and warmup (solid circles) under a fixed magnetic field of $0.02 \mathrm{~T}$. The effect of light irradiation at $5 \mathrm{~K}$ is also shown by an arrow. (c) Magnetization curves at $5 \mathrm{~K}$ observed before (open circles) and after (solid circles) light irradiation. Solid line shows a theoretical curve. (d) Hall resistivity $\rho_{\text {Hall }}$ observed at $5 \mathrm{~K}$ before (dashed line) and after (solid line) light irradiation (Koshihara et al. 1997).

concentration indicates the possibility of control of magnetic properties isothermally by light irradiation, electric field, carrier injection, and all other means that change the carrier concentration in semiconductors. The concept of such devices was proposed already in 1960s in the context of work on rare-earth magnetic semiconductors (Methfessel 1965, Methfessel and Holtzberg 1966).

(In,Mn)As (12 nm, $x=0.06) / \mathrm{GaSb}$ heterostructure was found to exhibit photo-induced ferromagnetism as shown in Fig. 37 (Koshihara et al. 1997). The illumination by photons with the energy larger than the bandgap of GaSb $(\sim 0.8 \mathrm{eV})$ turns the paramagnetic sample without remanent magnetization to a ferromagnetic state with a clear hysteresis at $5 \mathrm{~K}$, which is documented by both magnetization and magnetotransport measurements. The ferromagnetic state persists even after switching the light off and, at the same time, a persistent con ductivity is observed. If the sample is heated up to $\sim 45 \mathrm{~K}$, the initial state is recovered. It appears that the interface electric field separates photo-holes and photo-electrons, the former being accumulated in the (In,Mn)As layer, which triggers the ferromagnetism. According to the Hall measurements up to $15 \mathrm{~T}$, a critical hole-concentration that generates the ferromagnetic order is $\sim 3.8 \times 10^{19} \mathrm{~cm}^{-3}$, whereas the change of the hole concentration after illumination is as low as $1.4 \times 10^{18} \mathrm{~cm}^{-3}$. 
Further studies are necessary in order to clarify the reason why there is a threshold hole concentration and why only a slight increase of the hole concentration leads to such a dramatic effect. A similar persistent light-enhanced magnetization is observed in a $\left(\mathrm{In}_{0.95} \mathrm{Mn}_{0.05}\right)\left(\mathrm{As}_{0.8} \mathrm{Sb}_{0.2}\right) / \mathrm{InSb}$ heterostructure (Kanamura et al. 2002). Reflecting the small band-gap of InSb, a lower photon energy is more efficient than that for the (In,Mn)As case. On the other hand, it is difficult to observe irradiation effects in $\left(\mathrm{In}_{0.95} \mathrm{Mn}_{0.05}\right)\left(\mathrm{As}_{0.2} \mathrm{Sb}_{0.8}\right) / \mathrm{InSb}$, which may be related to the small built-in electric field in the structure.

Ferromagnetic (In,Mn)As/GaSb heterostructures with rectangular hysteresis show also peculiar light-irradiation effects. In particular, the coercive force is drastically reduced by the illumination, which suggests a reduction of the domain wall pining energy (Oiwa et al. 2001).

The magnetization enhancement of (Ga,Mn)As by circularly polarized light illumination has been also observed (Oiwa et al. 2002).

These effects open up the possibility of novel magneto-optical memory devices.

9.6. Electric-field control of ferromagnetism in gated structures

The modification of ferromagnetism was demonstrated also in a structure consisting of an insulating-gate field-effect transistor (FET) with an (In,Mn)As channel (Ohno et al. 2000). The 5-nm thick channel layer $(x=0.03)$ was grown o $\mathrm{n}$ a $10 \mathrm{~nm} \mathrm{InAs} / 500 \mathrm{~nm}(\mathrm{Al}, \mathrm{Ga}) \mathrm{Sb}$ buffer and a GaAs substrate. A $0.8 \mu \mathrm{m}$ gate insulator and a metal electrode completed the device. The hole concentration in the channel was estimated to be $5 \sim 8 \times 10^{13} \mathrm{~cm}^{-2}$ from the resistance changes with the gate voltage and Hall effect at room temperature. Figure 38 shows magnetization deduced from the Hall resistance at $22.5 \mathrm{~K}$ for three different values of the gate voltage $V_{\mathrm{G}},+125,0$, and $-125 \mathrm{~V}$. The bias of $V_{\mathrm{G}}=125 \mathrm{~V}$ changes the sheet-hole concentration by $\sim 3 \times 10^{12} \mathrm{~cm}^{-2}$. At zero gate bias, the channel is weakly ferromagnetic as can be seen from the presence of a small hysteresis. The application of a positive gate voltage depletes the channel and, thus, reduces the ferromagnetic interaction mediated by the holes, which results in a paramagnetic behavior of the magnetization without hysteresis. When the holes are accumulated by applying a negative gate voltage, a clear hysteresis appears. The magnetization curve resumes its original values as the gate voltage returns to $0 \mathrm{~V}$. The $125 \mathrm{~V}$ swing give rise to $\pm 6 \%$ change in the hole concentration and results in a $T_{\mathrm{C}}$ change of $\pm 4 \%$ ( $\pm 1 \mathrm{~K}$ ).

The value of $T_{\mathrm{C}}$ calculated form the mean-field theory for three-dimensional (3D) case (Dietl et al. 2000, 2001c) is $\sim 18 \mathrm{~K}$ for (In,Mn)As with $x=0.03$ and the hole density $1 \times 10^{20} \mathrm{~cm}^{-3}$, which corresponds to $p=5 \times 10^{13} \mathrm{~cm}^{-3}$ in a 5 nm-thick film. For this calculation the Luttinger-Kohn parameters given in by Wiley (1975), the energy of the exchange interaction between the holes and Mn spins $N_{0} \beta=$ $-0.98 \mathrm{eV}$ (Dietl et al. 2001d), as well as the enhancement factor $A_{\mathrm{F}}=1.2$ describing carrier-carrier interactions (Jungwirth et al. 1999) are adopted. The calculation also shows that a modulation of $\Delta p=3 \times 10^{12} \mathrm{~cm}^{-2}$ gives a change of $T_{\mathrm{C}}$ of $\sim 1 \mathrm{~K}$. These evaluations show, therefore, a rather good correspondence with the experimental findings. On the other hand, the thickness of the $(\mathrm{In}, \mathrm{Mn}) \mathrm{As}$ channel in the examined FET structures may

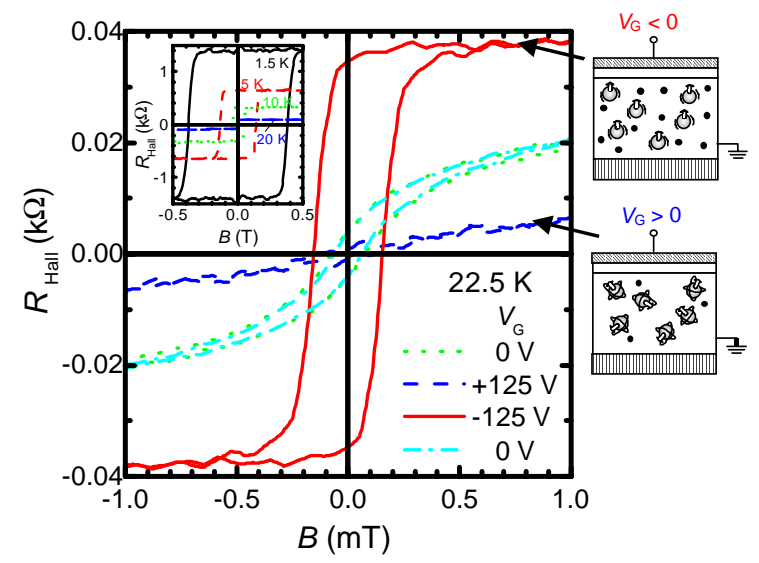

Fig. 38. Hall resistance $R_{\text {Hall }}$ of an insulated gate (In,Mn)As field-effect transistor at $22.5 \mathrm{~K}$ as a function of the magnetic field for three different gate voltages. $R_{\text {Hall }}$ is proportional to the magnetization of the (In,Mn)As channel. Upper right inset shows the temperature dependence of $R_{\text {Hall }}$. Left inset shows schematically the gate voltage control of the hole concentration and the corresponding change of the magnetic phase. (Ohno et al. 2000).

suggest that the system is two-dimensional (2D). For 2D systems, the mean-field theory predicts no carrier concentration dependence of $T_{\mathrm{C}}$ as long as the $2 \mathrm{D}$ density-of-states (DOS) that determines $T_{\mathrm{C}}$ is energy-independent (Dietl et al. 1997). For the hole concentrations in question, several 2D subbands are occupied and the valence band DOS is a complex function of energy, so that we expect a modulation of $T_{\mathrm{C}}$ through a modulation of $p$. Moreover, since the mean free path is comparable to the quantum well width, the disorder-induced mixing of electric subbands turns DOS towards the $3 \mathrm{D}$ value (Kossacki et al. 2000).

An additional contribution to the modulation of $T_{\mathrm{C}}$ may come from the particular design of the FET device: a nonmagnetic InAs is placed beneath the magnetic (In,Mn)As layer. Application of positive (negative) bias displaces the hole wavefunctions away from (towards) the magnetic layer, resulting in a reduction (increase) of the interaction between the holes and magnetic spins and hence in reduction (increase) of $T_{\mathrm{C}}$ (Lee et al. 2000).

Control of ferromagnetism in gated structure has also been observed in a group VI ferromagnetic semiconductor $\mathrm{Mn}_{x} \mathrm{Ge}_{1-x}$ (Park et al. 2002).

\subsection{Ferromagnetic imprinting}

The manipulation of spin coherence of electron spins and nuclear spins in semiconductor materials is one of the most important issues to pursue in spintronics for spin-based qubits in future quantum computing. A proximity effect on spin coherence in nonmagnetic semiconductors in ferromagnet/semiconductor heterostructures has been observed (Kawakami et al. 2001). The spin dynamics in GaAs on which a ferromagnetic layer, (Ga,Mn)As or $\mathrm{MnAs}$, is grown, is investigated using time resolved Faraday rotation (TRFR). The results at $5 \mathrm{~K}$ show that the presence of a ferromagnetic layer strongly modifies the spin coherence of electrons. The dependence of TRFR on applied magnetic field shows hysteresis behavior, and the effective field extracted from the data suggests that there is a nuclei-mediated effective field. The 
results derived from all-optical nuclear magnetic resonance (Kikkawa and Awshalom 2000) on the samples also support the existence of the "imprinting" on nuclear spins by the neighboring ferromagnetic layer.

\section{Ferromagnetic semiconductors at room temperature}

For the application of magnetic semiconductors, a $T_{\mathrm{C}}$ above room temperature is required. Room-temperature ferromagnetic sulphospinels have been found in 1960's (Goodenough 1969, Van Stapele 1982), but the application of these ferromagnetic semiconductors has been hampered by difficulties in their fabrication. Recent experimental as well as theoretical progress in material science allows one to design and synthesize new ferromagnetic semiconductors with $T_{\mathrm{C}}$ above room temperature.

\subsection{Theoretical suggestions}

The mean-filed Zener model described in section 8 predicts $T_{\mathrm{C}}$ to lie above room temperature for DMS containing large amounts of magnetic ions and carriers. Thus, the elaboration of methods enabling a simultaneous increase of the magnetic ion and carrier concentrations in DMS is one of the ways to be pursued (Dietl et al. 2000, 2001c). Furthermore, a chemical trend was theoretically established, suggesting that the highest values of $T_{\mathrm{C}}$ can be achieved in materials containing light anions (Dietl et al. 2000, 2001c). The tendency for higher $T_{\mathrm{C}}$ values in the case of lighter elements stems from the corresponding increase in the $\mathrm{p}$-d hybridization and the reduction of the spin-orbit coupling. It can be expected that this tendency is not altered by the uncertainties in the values of the parameters employed for the computation. In particular, if one could introduce $5 \%$ of $\mathrm{Mn}$ and $3.5 \times 10^{20} \mathrm{~cm}^{-3}$ of holes into wide-gap semiconductors, such as $\mathrm{GaN}, \mathrm{ZnO}$, and $\mathrm{C}$, these materials should be ferromagnetic at room temperature. The first-principle calculation also predicts a rather stable ferromagnetism for these materials. The results show that $\mathrm{V}, \mathrm{Cr}, \mathrm{Fe}, \mathrm{Co}$, or Ni doped $\mathrm{ZnO}$ is a half-metallic double-exchange ferromagnet, $\mathrm{Mn}$ doped $\mathrm{ZnO}$ is an antiferromagnetic insulator which changes to a ferromagnet by additional doping of holes, whereas $\mathrm{Ti}$ or $\mathrm{Cu}$ doped $\mathrm{ZnO}$ remains paramagnetic (Sato and Katayama-Yoshida 2000). It is also predicted from the first-principle calculation that $\mathrm{V}-, \mathrm{Cr}-$, or Mn-doped $\mathrm{GaN}$ is ferromagnetic without additonal doping (Sato and Katayama-Yoshida 2001c).

In order to solve the problems of the low solubility of magnetic ions in III-V semiconductors and the difficulty of the carrier control in II-VI semiconductors, modulation-doped III-V/II-VI superlattice structures have been proposed (Kamatani and Akai 2001a). Here, a II-VI layer serves as the magnetic layer, which can contain a large concentration of $\mathrm{Mn}$, and III-V barrier layers, where impurities are incorporated, provide carriers to II-VI magnetic layers. The results of the first principle calculation for a $\mathrm{AlAs}: \mathrm{C} /(\mathrm{Cd}, \mathrm{Mn}) \mathrm{Te}$ superlattice shows that the ferromagnetic state is indeed stable for high concentrations of $\mathrm{C}$.

The highest spin and the associated large magnitude of the on-site correlation energy $U$ account for the divalent character of the Mn atoms in a large variety of environments.
This results, in particular, in a large solubility of $\mathrm{Mn}$ in II-VI materials and its acceptor character in a number of III-V compounds. A question arises about ferromagnetic properties of semiconductors containing other magnetic components. One should recall in this context the existence of, e.g., ferromagnetic europium chalcogenides and chromium spinels. In those compounds, the ferromagnetism is not driven by free carriers. With no doubt, the availability of intrinsic and n-type tetrahedrally-coordinated ferromagnetic compounds would enlarge considerably the impact of semiconductor electronics.

Actually, a theoretical suggestion was made that superexchange in Cr-based II-VI compounds can lead to ferromagnetic order (Blinowski et al. 1996). High composition (10 25\%) of $\mathrm{V}$ or $\mathrm{Cr}$ doped $\mathrm{ZnS}, \mathrm{ZnSe}$, and $\mathrm{ZnTe}$ have been predicted by the first-principle calculations to exhibit ferromagnetism at room temperature even without $\mathrm{p}$ - or n-type doping (Sato and Katayama-Yoshida, 2001a). Desirable material properties, such as divergent magnetic susceptibility and spontaneous magnetization, can also be achieved in the case of a strong antiferromagnetic super-exchange interaction. The idea here (Dietl 1994) is to synthesize a ferrimagnetic system that would consist of antiferromagnetically coupled alternating layers containing different magnetic cations, e.g., Mn and Co.

In general terms, the transition metals (TM) other than $\mathrm{Mn}$ are no longer always divalent, they lead to the presence of magnetic levels in the gap, and they are characterized by a non-zero orbital momentum. These will considerably modify transport and optical properties as well as enhance the sensitivity to co-doping and illumination. Furthermore, an increase in magnetic anisotropy as well as an enlargement of the coupling to phonons and thus a shortening of spin-lattice relaxation time are expected too. Recent ab initio calculations of Sato and Katayama-Yoshida (2000) suggest that $\mathrm{V}, \mathrm{Cr}, \mathrm{Fe}, \mathrm{Co}$, and $\mathrm{Ni}$ in $\mathrm{ZnO}$ should provide carriers, which owing to the double exchange mechanism generate ferromagnetic order. We note that in the case of wide gap II-VI compounds studied so far, neither of these transition metals (TM) introduces free carriers. However, for sufficiently high TM concentrations a Mott-Hubbard transition is expected, leading to a transport through the gap d-states. A co-doping with either shallow acceptors or donors could make such transport, and the associated exchange interaction, more efficient. Since the TMs act as deep donors and acceptors, the co-doping of such compounds with shallow impurities (e.g., by $\mathrm{O}$ for $\mathrm{Mn}$ in III-V compounds) constitutes a natural way to control the ferromagnetic couplings (Katayama-Yoshida et al. 2001). Indeed, according to Sato and Katayama-Yoshida (2001b), for $\mathrm{Fe}$, Co, or Ni doped $\mathrm{ZnO}$, the ferromagnetic state is stabilized by the doping of shallow donors.

\subsection{Cautionary remarks}

Before discussing experimental results, it is appropriate to enlist difficulties encountered in assessing properties of new ferromagnetic semiconductors. The well -known difficulty is the multi-phase character of materials grown by non-equilibrium techniques, such as MBE. In particular, some phases may consist of ferromagnetic and/or ferromagnetic precipitations, such as metallic and ferromagnetic MnAs. These precipitations, even if too small to be detected by 
x-ray diffraction, can give the dominant contribution to the total magnetic moment of the sample, particularly at high temperatures. Importantly, the Curie temperature of the precipitations may not be identical to the tabulated values, and may depend on their size and the host material. Another source of undesirable magnetic signals originates often from magnetic impurities residing outside the studied layer, for instance in the substrate. Since ferromagnetic semiconductors are characterized by colossal magnetotransport and magnetooptical phenomena, a correlation between semiconductor and magnetic properties allows one usually to rule out parasitic effects. However, stray magnetic fields produced by ferromagnetic inclusions or their contribution to charge transport may constitute a source of ambiguity. Additionally, multi-layer and/or multi-carrier transport of semiconductor structures, together with localization, surface, and interface phenomena, may generate strong magnetoresistance effects, usually hard to separate from spin-related phenomena in the examined material.

\subsection{Experimental results}

\subsection{1 (Ga,Mn)As}

As mentioned above, the mean-field Zener model suggests that $T_{\mathrm{C}}$ values above $300 \mathrm{~K}$ could be achieved in, e.g., $\mathrm{Ga}_{0.9} \mathrm{Mn}_{0.1} \mathrm{As}$, if such a large value of $x$ would be accompanied by a corresponding increase of the hole concentration. The elaboration of an annealing procedure that increases $T_{\mathrm{C}}$ (Katsumoto et al. 1999, Poshtanik et al. 2001) as well as a successful implementation of low-temperature atomic layer epitaxy to increase the Mn composition $x$ (Sadowski et al. 2001a) constitute examples of recent encouraging developments in this direction.

\subsection{2 (Ga,Mn)N}

As discussed in section 2.4, indications of high temperature ferromagnetism in $(\mathrm{Ga}, \mathrm{Mn}) \mathrm{N}$ have been reported by Sonoda et al. (2001) and Reed et al. (2001), whose layers grown by ammonia-MBE or prepared by solid state diffusion show ferromagnetism well above room temperature. Work is under way to rule out the influence of precipitations as well as to establish how $T_{\mathrm{C}}$ depends on the $\mathrm{Mn}$ and carrier concentration. Possible mechanisms accounting for the experimental observations have been put forward (Dietl et al. 2001b).

\subsection{3. (Cd,Mn) $\mathrm{GeP}_{2}$}

II-VI-V2 chalcopyrite DMS, $\left(\mathrm{Cd}_{1-x} \mathrm{Mn}_{x}\right) \mathrm{GeP}_{2}$ was prepared by the solid phase chemical reaction. $\mathrm{Mn}$ vacuum deposition $(30 \mathrm{~nm})$ on a single crystal of $\mathrm{CdGeP}_{2}$ and the reacting process $\left(500^{\circ} \mathrm{C}, 30 \mathrm{~min}\right)$ was carried out in an $\mathrm{MBE}$ chamber (Medvedkin et al. 2000). The $\mathrm{Mn} / \mathrm{Cd}$ composition ratio decreases rapidly with the depth. The average $\mathrm{Mn} / \mathrm{Cd}$ ratio was determined as $20 \%$ for an effective thickness $0.5 \mu \mathrm{m}$ by energy dispersive x-ray analysis. The value $T_{\mathrm{C}} \sim 320 \mathrm{~K}$ of $\left(\mathrm{Cd}_{1-x} \mathrm{Mn}_{x}\right) \mathrm{GeP}_{2}$ was determined by magnetization measurements. Clear hysteresis in the field dependence of magnetization and the stripe magnetic domain pattern were observed by magnetic force microscopy (MFM) even at room temperature. A large Faraday rotation of $5.7 \times 10^{4} \mathrm{deg} / \mathrm{cm}$ at an energy gap of $\mathrm{CdGeP}_{2}(1.83 \mathrm{eV})$ was estimated from the magneto-optical Kerr effect at room temperature. The energy gap of $(\mathrm{Cd}, \mathrm{Mn}) \mathrm{GeP}_{2}$ is two times larger than that of $\mathrm{CdGeP}_{2}$. Photoluminescence indicates also that the introduction of $\mathrm{Mn}$ enlarges the energy gap.

Results of a first principles calculation shows that the antiferromagnetic state is more stable than the ferromagnetic state, and that the energy gap decreases with the Mn composition (Zhao, Y.-J. et al. 2001b). The reason for the discrepancies between theoretical expectations and experimental results is not clear; it may stem from the substitution of Ge for $\mathrm{Mn}$ in surface-doped samples. More recent plane-wave pseudopotential and KKR-CPA calculations shows that the intrinsic defects are responsible for the stabilization of the ferromagnetic state (Mahadevan and Zunger 2002, Kamatani and Akai 2001b).

\subsubsection{Co-doped $\mathrm{TiO}_{2}$}

It has been shown by means of magnetization measurements that anatase and rutile (two forms of $\mathrm{TiO}_{2}$ ) doped with several percents of $\mathrm{Co}$ are ferromagnetic at room temperature (Matsumoto et al. 2001a, 2001b). Co-doped $\mathrm{TiO}_{2}$ has been synthesized by laser ablation MBE employing a combinatorial method, in which a series of thin films with different compositions can be grown on a single substrate while keeping other growth parameters virtually unchanged (Ohno 2001). No ferromagnetic order has been found for other form of $\mathrm{TiO}_{2}$ (blookite) and for other transition metals.

\subsubsection{Co doped $\mathrm{ZnO}$}

The magnetic properties of n-type transition-metal doped $\mathrm{ZnO}$ have been investigated (Ueda, K. et al. 2001). The material is synthesized by pulsed laser deposition, and $1 \%$ of $\mathrm{Al}$ is added to produce n-type conduction. $\mathrm{Co}, \mathrm{Ni}, \mathrm{Cr}$, or $\mathrm{Mn}$ serve as a source of the magnetic spins. $\mathrm{ZnO}$ with $5 \%$ of $\mathrm{Mn}, \mathrm{Ni}$, or Co shows an antiferromagnetic behavior with Néel temperatures of 310,350 , and $270 \mathrm{~K}$, respectively. On the other hand $\mathrm{ZnO}$ with $5-25 \%$ of Co exhibits ferromagnetic (or weak-ferromagnetic) behavior, which is confirmed by magnetization measurements. The magnetic properties depend on carrier concentration and mobility. Samples with higher carrier concentrations and mobilities show stable ferromagnetism, otherwise spin-glass behavior is observed.

$\mathrm{V}$ doped $\mathrm{ZnO}$ (V composition 0.05 0.15) with higher conductivity shows ferromagnetic behavior at room temperature, whereas that with lower conductivity is nonmagnetic (Saeki et al. 2001).

\subsubsection{Zinc-blende $\mathrm{CrAs}$ and $\mathrm{CrSb}$}

Thin epitaxial films (less than $3 \mathrm{~nm}$ ) of CrAs and $\mathrm{CrSb}$ with zinc-blende structure can be grown on GaAs substrates by MBE. Their $T_{\mathrm{C}}$ exceeds $400 \mathrm{~K}$ (Akinaga et al. 2000c, Zhao et al. 2001b). A zinc-blende structure is confirmed by in-situ RHEED collected during the growth and ex-situ cross-sectional transmission electron microscopy (TEM). The preparation and properties of zinc-blende MnAs have also been 
investigated (Okabayshi et al. 2001d, Hazama et al. 2001). According to first-principle calculations (Shirai 2001) such materials possess a half-metallic electronic structure. Hence, being compatible with the existing semiconductor electronics, these systems appear to be promising spin injectors.

\section{Summary and outlook}

Ferromagnetic semiconductors based on III-V compounds can be incorporated into III-V based epitaxial structures allowing one to explore spin-dependent phenomena, not available in structures made of nonmagnetic semiconductors alone. There are two major directions for the exploration of spin-dependent phenomena in magnetic semiconductors. One concerns with new functionalities and materials for classical devices, such as optical isolators and modulators, magnetic sensors and memories. This direction requires systems with $T_{\mathrm{C}}$ above room temperature. The other direction is quantum related: new developments in magnetic III-V heterostructures combined with recent progress in coherent manipulation of carriers (Kikkawa and Awschalom, 1999, Malajovich et al. 2000, 2001, Salis et al. 2001b) and nuclear spins (Kikkawa and Awschalom 2000, Salis et al. 2001a) pave the way for future quantum information technologies that will utilize spins in semiconductors (Kane 1998, Loss and DiVincenzo 1998, Das Sarma et al. 2000, Vrijen et al. 2000). Ferromagnetic III-V heterostructures are excellent media to explore this new field of semiconductor physics and technology, where both charge and spins play critical roles. With no doubt, however, there is plenty of room for new ideas how to explore outstanding properties of magnetic semiconductors, which have not yet been exploited.

\section{Acknowledgements}

The authors thank many collaborators for fruitful discussion, especially colleagues at Tohoku University. The work at Tohoku University was supported partly by the Japan Society for the Promotion and Ministry of Education, Culture, Sports, Science and Technology, Japan, and the work at Polish Academy of Sciences was supported partly by Foundation for Polish Science.

\section{References}

Abe, E., F. Matsukura, H. Yasuda, Y. Ohno and H. Ohno, 2000, Physica E 7, 981 .

Abe, E. K. Sato, F. Matsukura, Y. Ohno and H. Ohno, 2001, Extend Abstracts (The 48th Spring Meeting, The Japan Society of Applied Physics), p.1322.

Abolfath, M., T. Jungwirth, J. Brum and A. H. MacDonald, 2001, Phys. Rev. B 63, 054418 .

Adhikari T and S, Basu, 1984, Jpn. J. Appl. Phys., Part I, 33, 4581.

Akai, H., 1998, Phys. Rev. Lett. 81, 3002.

Akiba, N., F. Matsukura, A. Shen, Y. Ohno, H. Ohno, A. Oiwa, S. Katsumoto and Y. Iye, 1998a, Appl. Phys. Lett. 73, 2122.

Akiba, N., F. Matsukura, Y. Ohno, A. Shen, K. Ohtani, T. Sakon, M. Motokawa and H. Ohno, 1998b, Physica B 256-258, 561.

Akiba, N., D. Chiba, K. Nakata, F. Matsukura, Y. Ohno and H. Ohno, 2000a, J. Appl. Phys. 87, 6436.

Akiba, N., F. Matsukura, T. Dietl, K. Ohtani, Y. Ohno and H. Ohno, 2000b, presented at the Int. Conf. on Physics and Application of Spin-Related Phenomena in Semiconductors, Sendai, Japan, Sep.
$13-15,2000$.

Akinaga, H., S. Basu, et al., 2000a, presented at Fall Meeting, The Japan Society of Applied Physics, Sapporo, Sep. 2000.

Akinaga, H., S. Németh, J. De Boeck, H. Bender, G. Borghs, H. Ofuchi and M. Oshima, 2000b, Appl. Phys. Lett. 77, 4377.

Akinaga, H., T. Manago and M. Shirai, 2000c, Jpn. J. Appl. Phys. Part 2, 39, L1118.

Aliyev, M. I., I. S. Dadashev and G. I. Safaraliyev, 1980, Phys. Met. Metallogr. 49, 166.

Almesh, N. and B. Goldstein, 1962, Phys. Rev. 128, 1568.

Al'tshuler, B. L. and A. G. Aronov, 1985, in Electron-electron interaction in disordered conductors (North-Holland, Netherlands), pp. $1-153$.

Ando, K. T. Hayashi, M. Tanaka and A. Twardowski, 1998, J. Appl. Phys. 83, 6548.

Ando, M, Y. Kanemitsu, K. Takamura, F. Matsukura and H. Ohno, 1999 Fifth Symposium on Physics and Application of Spin-Related Phenomena in Semiconductors, 16-17 Dec. 1999, Sendai, Japan, p. 190 .

Arata, I., Y. Ohno, F. Matsukura and H. Ohno, 2001, Physica E 10, 288.

Arrott, A., 1957, Phys. Rev. 108, 1394.

Ball, P., Nature 404, 918 (2000).

Baron, T., S. Tatarenko, K. Saminadayer, N. Magnea, and J. Fontenille, 1994, Appl. Phys. Lett. 65. 1284.

Baxter, D. V., D. Ruzmetov, J. Scherschligt, Y. Sasaki, X. Liu, J. K. Furdyna, and C. H. Mielke, cond-mat/0202508, submitted to Phys. Rev. B

Belitz, D. and T. R. Kirkpatrick, 1994, Rev. Mod. Phys. 57, 287.

Benoit à la Guillaume, C., D. Scalbert and T. Dietl, 1992, Phys. Rev. B 46, 9853.

Beschoten, B., P. A. Crowell, I. Malajovich, D. D. Awshalom, F. Mastukura, A. Shen and H. Ohno, 1999, Phys. Rev. Lett. 83, 2073.

Bhatt, R. N. and X. Wan, 1999 Intern. J. Modern. Phys. C 10, 1459.

Bhatt, R. N. and M. Berciu, 2001, Phys. Rev. Lett. 87, 10723.

Bhattacharjee, A. K. and C. Benoit à la Guillaume, 2000, Solid State Commun. 113, 17.

Bither, T. A. and W.H. Cloud, 1965, J. Appl. Phys. 36, 1501.

Blinowski, J., P. Kacman and J. A. Majewski, 1996, Phys. Rev. B 53, 9524.

Blinowski, J., P. Kacman and J. A. Majewski, 1997, in High Magnetic Fields in Semiconductor Physics II, edited by G. Landwehr and W. Ossau (World Scientific, Singapore) p. 861.

Bosselli, M. A., A. Ghazali and I. C. da Cunha Lima, 2000, Phys. Rev. B 62, 8895 .

Casey, H. C., Jr., D. D. Sell and K. W. Wecht, 1975, J. Appl. Phys. 46, 250

Chao, C. Y. -P. and S. L. Chuang, 1991, Phys. Rev. 43, 7027.

Chapman, R. A. and W. G. Hutchinson, 1967, Phys. Rev. Lett. 18, 443.

Chattopadhyay, A., S. Das Sarma and A. J. Millis, 2001, Phys. Rev. Lett. 87, 227202.

Chazalviel, J. -N., 1975, Phys. Rev. B 11, 3918.

Chen, X., K. P. Mooney, G. Comenescu, L. Guo, B. D. McCombe, Y. Sasaki, X. Liu, S. J. Poteshnik, P. Sciffer and J. K. Furdyna, 2000, APS March 2000 Meeting, 20-24 Mar. 2000, Minneapolis, USA.

Chiba, D., N. Akiba, F. Matsukura, Y. Ohno and H. Ohno, 2000, Appl. Phys. Lett. 77, 1873.

Chien, C. L. and C. W. Westgate, 1980, The Hall effect and Its Applications, Plenum Press, New York.

Coey, J. M. D., M. Viret and S. von Molnár, 1999, Adv. Phys. 48, 167.

Crépieux, A. and P. Bruno, 2001, cond-mat/0101376.

Das Sarma, S., J. Fabian, X. Hu and I. Zutic, 2000, Superlattice and Microst. 27, 285.

De Boeck, J., R. Oesterholt, A. Van Esch, H. Bender, C. Bruynseraede, C. Van Hoof and G. Borghs, 1996, Appl. Phys. Lett. 68. 2744.

De Boeck, J. and G. Borghs, 1999, Phys. World, No 4, p. 27.

DeSimone, D., C. E. C. Wood, C. A. Evans Jr., 1982, J. Appl. Phys. 53, 4938.

Dietl, T., 1994, (Diluted) Magnetic Semiconductors, in: T. S. Moss (Ed.), Handbook of Semiconductors, Vol. 3B, North-Hoalland, Amsterdam.

Dietl, T., A. Haury and Y. Merle d'Aubigné, 1997, Phys. Rev. B 55, R3347.

Dietl, T, J. Cibert, D. Ferrand and Y. Merle d'Aubigné, 1999, Mater. Sci. Eng. B 63, 103.

Dietl, T., H. Ohno, F. Matsukura, J. Cibert and D. Ferrand, 2000, Science 287, 1019

Dietl, T., J. König and A. H. MacDonald, 2001a, cond-mat/0107009, Phys. Rev. B 64, 241201.

Dietl, T., F. Matsukura, and H. Ohno, 2001b, cond-mat/0109145.

Dietl, T., H. Ohno and F. Matsukura, 2001c, Phys. Rev. 63, 195205. 
Dorlrijn, J. W. F., 1976, Philips Res. Repts. 31, 287.

Edwards, P. P. and M. J. Sienko, 1978, Phys. Rev. B 17, 2575.

Edwards, P. P. and C.N.R. Rao (eds.), 1995, Metal-Insulator Transitions Revisited (Taylor and Francis, London)

Eggenkamp, P. T. J., H. J. M. Swagten, T. Story, V. I. Litvinov, C. H. W. Swuste and W. J. M. de Jonge, 1995, Phys. Rev. B 51, 15250.

Endo, T. T. Slupinski, S. Yanagi, A. Oiwa and H. Munekata, 2001, Seventh Symposium on the Physics and Application of Spin-Related Phenomena in Semiconductors, 17-18 Dec 2001, Yokohama, Japan, p. 33.

Fedorych, O. M., E. M. Hankiewicz, Z. Wilamowski and J. Sadowski, 2001, cond-mat/0106227 (J. Magn. Magn. Mater.).

Fernández-Rossier, J. and L. J. Sham, 2001, Phys. Rev. B 64, 235323

Ferrand, D., J. Cibert, A. Wasiela, C. Bourgognon, S. Tatarenko, G. Fishman, S. Koleśnik, J. Jaroszyński, T. Dietl, B. Barabara and D. Dufeu, 2000, J. Appl. Phys. 87, 6451.

Ferrand, D., J. Cibert, A. Wasiela, C. Bpurgognon, S. Tatarenko, G. Fishman, T. Andrearczyk, J. Jaroszyński, S. Koleśnik, T. Dietl, B. Barbara and D. Dufeu, 2001, Phys. Rev. B 63, 085201.

Fiederling, R., M. Kelm, G. Reuscher, W. Ossau, G. Scmidt, A. Waag and L. W. Molenkamp, 1999, Nature 402, 787.

Fisher, M. E., S. -k. Ma and B. G. Nickel, 1972, Phys. Rev. Lett. 29 , 917.

Frölich, H. and F. R. N. Nabarro, 1940, Proc. R. Soc. London Ser. A 175, 382.

Fukumura, T., T. Shono, K. Inaba, T. Hasegawa, H. Koinuma, F. Matsukura and H. Ohno, 2001, Physica E 10, 135.

Fumagalli, P. and H. Munekata, 1996, Phys. Rev. B 53, 15045.

Furdyna, J. and J. Kossut (eds.), 1988, Semiconductor and Semimetals, Vol. 25, Academic, New York.

Gaj, J. A., R. Planel and G. Fishman, 1979, Solid State Commun. 29 435.

Gałązka, R. R. 1977, Postępy Fyzyki 28, 601.

Gaymann, A., H. P. Geserich and H. v. Löhneysen, 1995, Phys. Rev. B 52, 16486.

Gębicki, W., J. Strzeszewski, G. Kalmer, T. Szyszko and S. Podsiadło, 2000, Appl. Phys. Lett. 76, 3870.

Głód, P., T. Dietl, M. Sawicki and I. Miotkowski, 1994, Physics B 194-196, 995.

Goodenough, J. B., J. Phys, Chem. Solids. 30, 261

Grandidier, B., J. P. Nys, C. Delerue, D. Stiévenard, Y. Higo and M. Tanaka, 2000, Appl. Phys. Lett. 77, 4001.

Guha, S. and H. Munekata, 1993, J. Appl. Phys. 74, 2975

Gummich, U. and I. C. da Cunha Lima, 1990, Solid State Commun. 76 831

Guo, S. P., H. Ohno, A. Shen, F. Matsukura and Y. Ohno, 1998, Appl. Surf. Sci. 130-132, 797

Guo, S. P., A. Shen, F. Matsukura, Y. Ohno and H. Ohno, 1999, J. Cryst. Growth, 201/202, 684-688.

Guo, S. P, A. Shen, H. Yasuda, Y. Ohno, F. Matsukura, and H. Ohno, 2000, J. Cryst. Growth 208, 799.

Haneda, S., M. Yamamura, Y. Takatani, K. Hara, S. Harigae and H. Munekata, 2000a, Jpn. J. Appl. Phys. 39, L9.

Haneda, S., H. Munekata, Y. Takatani and S. Koshihara, 2000b, J. Appl. Phys. 87, 6445.

Haneda, S., S. Kodhihara and H. Munekata, 2001, Physica E 10, 437.

Hansen, L., D. Ferrand, G. Richter, M. Thierley, V. Hock, N. Schwarz, G. Reuscher, G. Scmidt, A. Waag and L. W. Molenkamp, 2001, cond-mat/0107619; submitted to Appl. Phys. Lett.

Harris, J. G. E., D. D. Awshalom, F. Matsukura, H. Ohno, K. D. Maranowski and A. C. Gossard, 1999, Appl. Phys. Lett. 75, 1140.

Hartmann, Th, M. Lampalzer, W. Stolz, K. Megges, L. Lorberth, P. J. Klar and W. Heimbrodt, 2000, Thin Solid Films 364, 209.

Hashimoto, M., Y.-K. Zhou, M. Kanamura and H. Asahi, 2002, to be published in Solid State Commun.

Hashimoto, Y., T. Hayashi, S. Katsumoto and Y. Iye, 2002, J. Cryst. Growth, to be published.

Hass, K. C., in Semimagnetic Semicondictors and Diluted Magnetic Semiconductoes, edited by M. Averous and M. Balkanski (Plenum, London, 1991).

Haury, A., A. Wasiela, A. Arnoult, J. Cibert, S. Tatarenko, T. Dietl and Y Merle d'Aubigné, 1997, Phys. Rev. Lett. 79. 511.

Hayashi, T., M. Tanaka, T. Nishinaga, H. Shimada, H. Tsuchiya and Y. Ohtsuka, 1997a, J. Cryst. Growth 175/176. 1063.

Hayashi, T., M. Tanaka, K. Seto, T. Nishinaga and K. Ando, 1997b, Appl. Phys. Lett. 71, 1825 .

Hayashi, T., M. Tanaka, K. Seto, T. Nishinaga, H. Shimada and K. Ando, 1998, J. Appl. Phys. 83, 6551.

Hayashi, T., H. Shimada, H. Shimizu and M. Tanaka, 1999, J. Crystal Growth 201/202, 689 .
Hayashi, T., S. Katsumoto, Y. Hashimoto, A. Endo, M. Kawamura, M. Zalalutdinov and Y. Iye, 2000, Physica B 284-288, 1175.

Hayashi, T., Y. Hashimoto, S. Katsumoto and Y. Iye, 2001, Appl. Phys. Lett. 78, 1691

Hazama, Y., K. Yamamoto, A. Nagashima and J. Yoshino, 2001, Seventh Symposium on Physics and Application of Spin-Related Phenomena in Semiconductors, 17-18 Dec. 2001, Sendai, Japan, p. 160

Heimbrodt, W., Th. Hartmann, P. J. Klar, M. Lampalzer, W. Stolz, K.Volz, A. Schaper, W. Treutmann, H. -A. Krug von Nidda, A Loidl, T. Ruf and, V. F. Sapega, 2001, Physica E 10, 175.

Henriques, A. B, S. A. Obukhov, N. F. Yr. Oliveira and V. A. Sanina, 1999, Pis'ma v ZhETF 69, 358.

Higo, Y., H. Shimizu and M. Tanaka, 2001, Physica E 10, 292

Higo, Y. H. Shimizu and M. Tanaka, 2001, J. Appl. Phys. 89, 6745.

Hirakawa, K., A. Oiwa and H. Munekata, 2001, Physica E 10, 215.

J.E. Hirsch, 1999, Phys. Rev. Lett. 83, 1834.

Ilegems, M. R. Digle and L.W. Rupp Jr. 1975, J. Appl. Phys. 46, 3059.

Inoue, J., S. Nonoyama and H. Itoh, 2000, Phys. Rev. Lett. 85, 4610.

Johnston-Halperin, E., D. Lofgreen, R. K. Kawakami, D. K. Young, I. Coldren, A. C. Gossard and D. D. Awshalom, 2002, Phys. Rev. B 65 041306

Jonker, J. T., Y. D. Park, B. R. Bennett, H. D. Cheong, G. Kioseoglow and A. Petrou, 2000, Phys. Rev. B 62, 8180 .

Jungwirth, T., W. A. Atkinson, B. H. Lee and A. H. MacDolnald, 1999, Phys. Rev. B 59, 9818

Jungwirth, T., Q. Niu and A. H. MacDonald, 2001, cond-mat/0110484.

Kacman, P., 2001, Semicond. Sci. Technol. 16, R25.

Kamatani, T. and H. Akai, 2001a, Physica E 10, 157.

Kamatani, T. and H. Akai, 2001b, Seventh Symposium on Physics and Application of Spin-Related Phenomena in Semiconductors, 17-18 Dec. 2001, Sendai, Japan, p. 124

Kanamura, M. Y. K. Zhou, S. Okumura, K. Asami, M. Nakajima, H. Harima and H. Asahi, 2002, Jpn. J. Appl. Phys. 41, 1019.

Kane, B. E., 1998, Nature 393 (1998).

Kasuya, T. and A. Yanase, 1968, Rev. Mod. Phys. 40. 684

Katayama-Yoshida, H., R. Kato and T. Yamamoto, 2001, J. Cryst. Growth, 231, 428

Kato, R. and H. Katayama-Yoshida, 1999, Fifth Symposium on Physics and Application of Spin-Related Phenomena in Semiconductors, 16-17 Dec. 1997, Sendai, Japan, p.233

Katsumoto, S., 1999, unpublished.

Katsumoto, S., A. Oiwa, Y. Iye, H. Ohno, F. Matsukura, A. Shen and Y Sugawara, 1998, Phys. Status Solidi b 205, 115

Katsumoto, S., T. Hayashi, Y. Hashimoto, Y. Iyem Y. Ishiwata, M. Watanabe, R. Eguchi, T. Takeuchi, Y. Harada, S. Shin and K. Hirakawa, 2001, Mater. Sci. Eng. B 84, 88.

Kawakami, R. K., E. Hohnston-Halperin, L. F. Chen, M. Hanson, N. Guébels, J. S. Speck, A. C. Gossard, and D. D. Awshalom, 2000, Appl. Phys. Lett. 77, 2379.

Kawakami, R. K., Y. Kato, M. Hanson, I. Malajovich, J. M. Stephens, E. Johnston-Halperin, G. Salis, A. C. Gossard and D. D. Awshalom, 2001, Science 294, 131.

Kenett, M. P., M. Berciu and R.N. Bhatt, 2001, cond-mat/0102315.

Kępa, H., J. Kutner-Pielaszek, A. Twardowski, C. F. Majkrzak, J. Sadowski, T. Story and T. M. Giebultwicz, 2001, Phys, Rev. B 64 121302.

Kikkawa, J. M. and D. D. Awschalom, 1999, Nature 397, 139.

Kikkawa, J. M. and D. D. Awschalom, 2000, Science 287, 473.

Kohda, M., Y. Ohno, K. Takamura, F. Matsukura and H. Ohno, 2001, Jpn. J. Appl. Phys. 40, L1274.

König, J., H.-H. Lin and A. H. MacDonald, 2000, Phys. Rev. Lett. 84, 5628 .

König, J., H.-H. Lin and A. H. MacDonald, 2001, Physica 10, 139.

Koshihara, S., A. Oiwa, M. Hirasawa, S. Katsumoto, Y. Iye, C. Urano, H. Takagi and H. Munekata, 1997, Phys. Rev. Lett. 78, 4617.

Kossacki, P., D. Ferrand, A. Arnoult, J. Cibert, S. Tatarenko, A. Wasiela, Y. Merle d'Aubigné, K. Świątek, M. Sawicki, J. Wróbel, W. Bardyszewski and T. Dietl, 2000, Physica E 6, 709.

Kossut, J. and W. Dobrowolski, 1993, in: Buschow, K. H. J. (Ed.), Handbook of Magnetic Materials, Vol. 7 North-Hoalland, Amsterdam.

Kotani T., 2000, J. Phys. Cond. Matter 12, 2413.

Krebs, J. J. and G. H. Stauss, 1977, Phys. Rev. B 16, 971.

Kreisel, J. W. Ulrici, M. El-Metoui, A. -M. Vasson, A. Vasson and A. Gavaix, 1996, Phys. Rev. B 54, 10508.

Krol, A., Y. L. Soo, S. Huang, Z. H. Ming, Y. H. Kao, H. Munekata and L. L. Chang, 1993, Phys. Rev. B 47, 7187.

Kuivalainen, P, 2001, phys. stat. sol. (b) 227, 449

Kuivalainen, P and A. Hovinen, 2002, J. Phys. D 35, 48

Kulatov, E., H. Nakayama, H. Mariette, H. Ohta and Yu. A. Uspenskii, 
2001, unpublished.

Kuroiwa, T., T. Yasuda, F. Matsukura, A. Shen, Y. Ohno, Y. Segawa and H. Ohno, 1998, Electron. Lett. 34, 190.

Kuwabara, S., K. Ishii, S. Haneda, T. Kondo, and H. Munekata, 2001a, Physica E 10, 233.

Kuwabara, S., T. Kondo, T. Chikyow, P. Ahmet, and H. Munekata, 2001b, Jpn. J. Appl. Phys. 40, L727.

Lee, B., T. Jungwirth and A. H. MacDonald, Phys. Rev. B 61, 15606 (2000).

Leroux-Hugon, P., 1973, in New Developments in Semiconductors, eds. P. R. Walace, R. Harris and M. J. Zuckermann (Noordhoff, Leyden).

Leroux-Hugon, P. and A. Ghazali, 1972, J. Phys. C 5, 1072.

Linnarson, M., E. Janzen, B. Monemar, M. Kleverman and M. Thilderkvist, 1997, Phys. Rev. B 55, 6938.

Litvinov, V. I. and V. K. Dugaev, 2001, Phys. Rev. Lett. 86, 5593.

Liu, S., K. Karrai, F. Dunmore, H. D. Drew, R. Wilson and G. A. Thomas, 1993, Phys. Rev. B 48, 11394.

Liu, X., Y. Sasaki and J. K. Furdyna, 2001. Appl. Phys. Lett. 79, 2414.

Liu, X. C., D. Heiman, J. Hao and K. C. Hsieh, in: Proceedings International Conference on Physics of Semiconductors in High Magnetic Fields, ed. D. Heiman (World Scientific, Singapore, 1995) p. 658 .

Look, D. C., 1991. J. Appl. Phys. 70, 3148

Loss, D. and D. P. DiVincenzo, 1998, Phys. Rev. A 57, 120.

Louriero da Silva, M. A. Boselli, I. C. da Cunha Lima, X. F. Wang, A. Ghazali, 2001, Appl. Phys. Lett. 79, 3305.

Luysberg, M. H. Sohn, A. Prasad, P. Specht, Z. Liliental-Weber, E. R. Weber, J. Gebauer and R. Krause-Rehberg, 1998, J. Appl. Phys. 83, 561.

Lyu, P. and K. Moon, 2001, Phys. Rev.B 64, 035201.

Ma, S.-k, 1976, Modern Theory of Critical Phenomena (W.A. Benjamin, Reading).

Mac, W., A. Twardowski and A.M. Hennel, 1994, in: D.J. Lockwood (Ed.), Proceedings of the 22nd International Conference on Physics of Semiconductors, (World Scientific, Singapore, 1995) p. 1569.

Mahadevan, P and A. Zunger, 2001, Phys. Rev. Lett. 88, 047205.

Malajovich, I., J. M. Kikkawa, D. D. Awschalom, J. J. Berry and N. Samarth, 2000, Phys. Rev. Lett. 84, 1014.

Malajovich, I., J. J. Berry, N. Samarth, and D. D. Awschalom, 2001, Nature, 411, 770.

Mašek, J. and F. Máka, 2001, Acta Phys. Polon. A 100, 319.

Masterov, V.F., K.F. Shtelmakh and M.N. Barbashov, 1988, Fiz. Tech.

Poluprodn. 22, 654 [Sov. Phys. Semicond. 22, 408].

Matsuda, Y. H., H. Arimoto, N. Miura, A. Twardowski, H. Ohno, A. Shen and F. Matsukura, 1998, Physica B 256-258, 565.

Matsuda, Y. H., T. Ikaida, N. Miura, M.A. Zudov, J. Kono and H. Munekata, 2001, Physica E 10, 219; Matsuda, Y. H., T. Ikaida, N. Miura, Y. Hashimoto, S. Katsumoto, J. Kono, M. A. Zudov, G. D. Sanders, C. J. Stanton and H. Munekata, Seventh Symposium on the Physics and Application of Spin-Related Phenomena in Semiconductors, 17-18 Dec, 2001, Yokohama, Japan, p. 41..

Matsukura, F, 1997, unpublished.

Matsukura, F., A. Shen, Y. Sugawara, T. Omiya, Y. Ohno and H. Ohno, 1998a, presented at the 25th Int. Symp. Compound Semiconductors, 12-16 October 1998, Nara, Japan; Proc. 25th Int. Symp. Compound Semiconductors, Institute of Physics Conference Series, No. 162 (IOP Publishing Ltd, Bistrol, 1999) p. 547.

Matsukura, F., H. Ohno, A. Shen and Y. Sugawara, 1998b, Phys. Rev. B 57, R2037.

Matsumoto, Y., M. Murakami, T. Shono, T. Hasegawa, T. Fukumura, M. Kawasaki, P. Ahmet, T. Chikyow, S. Koshihara and H. Koinuma, 2001, Science 291, 854.

Matsumoto, Y., R. Takahashi, M. Murakami, T. Koida, X. -J. Fan, T. Hasegawa, T. Fukumura, M. Kawasaki, S. Koshihara and H. Koinuma, 2001, Jpn. J. Appl. Phys. 40, L1204.

Mauger, A. and C. Gotard, 1986, Phys. Rep. 141. 51

Medvedkin, G. A., T. Ishibashi, T. Nishi, K. Hayata, Y. Hasegawa and K. Sato, 2000, Jpn. J. Appl. Phys. 39, L949.

Methfessel, S., 1965, IEEE Trans. Mag. 1, 144.

Methfessel, S. J. and F. Holtzberg, 1966, US Patent 3,271,709.

Methfessel, S. and D. C. Mattis, 1968, in: H. P. J. Wijn ed. Encyclopedia of Physics Vol. XVIII/1 Magnetism, Magnetic Semiconductors (Springer-Verlag, Berlin, 1968) pp.389-562.

Mizokawa, T. and A. Fujimori, 1993, Phys. Rev. B 48, 14150.

Moriya, R., H. Munekata, T. Kondo and A. Oiwa, 2002, J. Cryst. Growth, to be published.

Munekata, H., 1995, Mater. Sci. Eng. B 31, 151.

Munekata, H., H. Ohno, S. von Molnár, A. Segmüller, L. L. Chang and L. Esaki, 1989, Phys, Rev. Lett. 56. 777.

Munekata, H., H. Ohno, S. von Molnár, A. Harwit, A. Segmüller and L.
L. Chang, 1990, J. Vac. Sci. Technol. B 8, 176.

Munekata H., H. Ohno, R. R. Ruf, R. J. Gambino, L. L. Chang, 1991, J. Cryst. Growth 111, 1011

Munekata H., T. Penny and L. L. Chang, 1992, Surf. Sci. 267, 342.

Munekata, H., A. Zaslavsky, P. Fumagalli and R. J. Gambino, 1993, Appl. Phys. Lett. 63, 2929.

Munekata, H., T. Abe, S. Koshihara, A. Oiwa, M. Hirasawa, S. Katsumoto, Y. Iye, C. Urano and H. Takagi, 1997, J. Appl. Phys. 81, 4862 .

Nagai, Y., T. Kunimoto, K. Nagasaka, H. Nojiri, M. Motokawa, F. Matsukura, T. Dietl and H. Ohno, 2001, Jpn. J. Appl. Phys. 40, 6231.

Nishikawa, Y., Y. Satoh and J. Yoshino, 1997, Second Symposium on Physics and Application of Spin Related Phenomena in Semiconductors, 27-28 Jan 1997, Sendai, Japan, p. 122.

Nojiri, H. M. Motokawa, S. Takeyama, F. Matsukura and H. Ohno, 1998 Physica B 256-258, 569.

Nonoyama, S. and J. Inoue, 2001, Physica E 10, 283.

Obukhov, S. A., 1993, Solid Stata Commun. 88, 255.

Obukhov, S. A., 1996, Proceedings of 23rd International Conference on the Physics of Semiconductors, pp. 99-102 (World Scientific, Singapore).

Obukhov, S. A. and N. I. Pepic, 1989, Solid State Commun. 70, 103.

Ofuchi, H., M. Oshima, M. Tabuchi, Y. Takeda, H. Akinaga, Š. Németh, J. De Boeck and G. Borghs, 2001a, Appl. Phys. Lett. 78, 2470.

Ofuchi, H., T. Kubo, M. Tabuchi, Y. Takeda, F. Matsukura, S. P. Guo, A. Shen and H. Ohno, 2001b, J. Appl. Phys. 89, 66.

Ogawa, T., M. Shirai, N. Suzuki and I. Kitagawa, 1999, J. Magn. Magn. Mater. 196-197, 428

Ohldag, H., V. Solinus, F. U. Hillebrecht, J. B. Goedkoop, M. Finazzi, F. Matsukura and H. Ohno, 2000, Appl. Phys. Lett. 76, 2928.

Ohno, H., 1998, Science 281, 951.

Ohno, H., 1999, J. Magn. Magn. Mater. 200, 110.

Ohno, H., 2001, Science, 291, 840.

Ohno, H., H. Munekata, S. von Molnár and L. L. Chang, 1991, J. Appl. Phys. 69, 6103.

Ohno, H., H, Munekata, T. Penny, S. von Molnár and L. L. Chang, 1992 Phys. Rev. Lett. 68. 2664.

Ohno, H., A. Shen, F. Matsukura, A. Oiwa, A. Endo, S. Katsumoto and Y. Iye, 1996a Appl. Phys. Lett. 69. 363.

Ohno, H., F. Matsukura, A. Shen, Y. Sugawara, A. Oiwa, A. Endo, S. Katsumoto and Y. Iye, 1996b, in: M. Scheffler and R. Zimmermann (Eds.), Proceedings of the 23rd International Conference on Physics of Semiconductors, World Scientific, Singapore, pp. 405-408.

Ohno, H., N. Akiba, F. Matsukura, A. Shen, K. Ohtani and Y. Ohno, 1998, Appl. Phys. Lett. 73, 363.

Ohno, H. D. Chiba, F. Matsukura, T. Omiya, E. Abe, T. Dietl, Y. Ohno, and K. Ohtani, 2000, Nature 408, 944.

Ohno, H. and F. Matsukura, 2001, Solid State Commun. 117, 179.

Ohno, H., F. Matsukura and Y. Ohno, 2001, Solid State Commun. 119, 281

Ohno, Y., D. K. Young, B. Beshoten, F. Matsukura, H. Ohno and D. D. Awschalom, 1999, Nature 402, 790.

Ohno, Y., I. Arata, F. Matsukura, K. Ohtani, S. Wang and H. Ohno, 2000 , Appl. Surf. Sci. 159/160, 308.

Ohno, Y., I. Arata, F. Matsukura and H. Ohno, 2001, Physica E, to be published.

Ohya, E., H. Shimizu, Y. Higo, J. Sun and M. Tanaka, 2002, Jpn. J. Appl. Phys. 41, L24.

Oiwa, A. S. Katsumoto, A. Endo, M. Hirasawa, Y. Iye, H. Ohno, F. Matsukura, A. Shen and Y. Sugawara, 1997, Solid State Commun. 103, 209.

Oiwa, A., S. Katsumoto, A. Endo, M. Hirasawa, Y. Iye, F. Matsukura, A Shen, Y. Sugawara and H. Ohno, 1998a, Physica B 249-251, 775.

Oiwa, A., S. Katsumoto, A. Endo, M. Hirasawa, Y. Iye, H. Ohno, F. Matsukura, A. Shen and Y. Sugawawa, 1998b, Phys. Status Solidi b 205, 115 .

Oiwa, A., A. Endo, S. Katsumoto, Y. Iye, H. Munekata and H. Ohno, 1999, Phys. Rev. B 59, 5826.

Oiwa, A., T. Słupinski and H. Munekata, 2001, Appl. Phys. Lett. 78, 518

Oiwa, A. Y. Mitsumori, R. Moriya, T. Slupinski and H. Munekata, 2002, Phys. Rev. Lett. 88, 137202 (2002)..

Okabayashi, J. A. Kimura, O. Rader, T. Mizokawa, A. Fujimori, T. Hayashi and M. Tanaka, 1998, Phys. Rev. B 58, R4211.

Okabayshi, J. A. Kimura, T. Mizokawa, A. Fujimori, T. Hayashi and M. Tanaka, 1999, Phys. Rev. B 59, R2486.

Okabayshi, J., A. Kimura, O. Rader, T. Mizokawa, A. Fujimori, T. Hayashi and M. Tanaka, 2001a, Physica E 10, 192.

Okabayashi, J., A. Kimura, O. Rader, T. Mizokawa, A. Fujimori, T. 
Hayshi and M. Tanaka, 2001b, Phys. Rev. B 64, 125304.

Okabayashi, J., K. Ono, T. Mano, M. Mizuguchi, H. Horiba, S. Nakazoe, T. Kihara, K. Nakamura, H. Kiwata, A. Fujimori and M. Oshima, 2001c, Extend Abstracts (The 48th Spring Meeting, The Japan Society of Applied Physics), p.1325.

Okabayashi, J., K. Ono, M. Mizuguchi, M. Yamada, A. Fujimori, M. Oshima and H. Akinaga, Seventh Symposium on Physics and Application of Spin-Related Phenomena in Semiconductors, 17-18 Dec. 2001, Yokohama, Japan, p. 147.

Okazawa, D., K. Yamamoto and J. Yoshino, Third Symposium on Physics and Application of Spin-Related Phenomena in Semiconductors, 16-17 Dec. 1997, Sendai, Japan, p.212.

Okazawa, D, K. Yamamoto, A. Nagashima and J. Yoshino, 2001, Physica E 10, 229.

Omiya, T., F. Matsukura, T. Dietl, Y. Ohno, T. Sakon, M. Motokawa and H. Ohno, 2000, Physica E 7, 976.

Omiya, T., F. Matsukura, A. Shem, Y. Ohno and H. Ohno, 2001, Physica E 10, 206..

Osinniy, V., A. Jedrzejczak, M. Arciszewska, W. Dobrowolski, T. Story and J. Sadowski, 2001, Acta Phys. Polon. A 100, 327.

Overberg, M. E., C. R. Abernathy, S. J. Pearton, N. A. Theordropoulou, K. T. MacCarthy and F. Hebard, 2001, Appl. Phys. Lett. 79, 1312.

Paalanen, M. A. and R. N. Bhatt, 1991, Physica B 169, 223.

Park, J. H., S. K. Kwon and B. I. Min, 2000, Physica B 281 \& 282, 703.

Park, Y. D., A. T. Habbicki, S. C. Erwin, C. S. Hellberg, J. M. Sullivan, J. E. Mattson, T. M. Ambrose, A. Willson, G. Spanos and B. T. Jonker, 2002, Science 295, 651.

Parmenter, R.H., 1973, Phys. Rev. B 8, 1273.

Pashitskii, E. A. and S. M. Ryabchenko, 1979, Fizika Tverdogo Tela, 21, 545.

Petukhov, A. G., D. O. Demchenko and A. N. Chantis, 2000, J. Vac. Sci. Technol. B 18, 2109.

Potashnik, S. J., K. C. Ku, S. H. Chun, J. J. Berry, N. Samarth and P. Schiffer, 2001, Appl. Phys. Lett. 79, 1495.

Reed, M. L., N. A. El-Masry, H. H. Stadelmaier, M. K. Ritums, M. J. Reed, C. A. Parker, J. C. Roberts and S. M. Bedair, 2001, Appl. Phys. Lett. 79, 3473.

Price, G. L., 1991, Phys. Rev. Lett. 66, 469.

Prinz, G.A., 1998, Science 282, 1660.

Rodrigues Bitterncourt, A. C., A. M. Cohen and G. E. Marques, 1998, Phys. Rev. B 67, 4525 .

Sadowski, J., J. Domagala, J. Bak-Misiuk, K. Sqiatek, J. Kanski, L. Ilver and H. Oscarsson, 1998, Acta Physica Polonica A 94, 509.

Sadowski, J., J. Z. Domagała, J. Bąk-Misiuk, S. Koleświcki, K. Śątek, J. Kanski, L. Ilver and J. Ström, 2000, J. Vac. Sci. Technol. B 18, 1697

Sadowski, J., P. Mathieu, P. Svedlindh, J. Z. Domagala, J. Bak-Misiuk, K. Swiatek, M. Karlsteen, J. Kanski, L. Ilver, H. Åsklund and U. Södervall, 2001a, Appl. Phys. Lett. 78, 3271.

Sadowski, J., P. Mathieu, P. Svedlindh, J M. Karlsteen, J. Kanski, L. Ilver, H. Åsklund, K. Świątek, J. Z. Domagała, J. Bąk-Misiuk, and D. Maude, 2001b, Physica E 10, 181.

Sadowski, J., E. Vanelle, D. S. Yee, D.Hrabovski, J. Kanski and L. Ilver, 2001c, presented at XXX International School on the Physics of Semiconducting Compounds "Jaszowiec 2001" June 1-8, 2001, Ustroń-Jaszowiec, Poland.

Sadowski, J. et al., 2001d, presented at Drugi Kongres Polskiego Towarzystwa Próżniowego, May 13-17, 2001, Warszawa, Poland.

Sadowski, J., K. Świątek, P. Svedlindh, R. Mathieu, M. Karlsteen, J. Kanski, L. Ilver, 2001e, submitted to Phys. Rev. B.

Saeki, H., H. Tabata and T. Kawai, 2001, Solid State Commun. 120, 439.

Saito, H, W. Zaets, R. Akimoto, K. Ando, Y. Mishima and M. Tanaka, 2001, J. Appl. Phys. 89, 7392.

Sakai, O., S. Suzuki and K. Nishizawa, 2001a, J. Phys. Soc. Jpn. 70, 1105 .

Sakai, O. and S. Suzuki, 2001b, Physica E 10, 148.

Salis, G., D. T. Fuchs, J. M. Kikkawa, D. D. Awshalom, Y. Ohno and H. Ohno, 2001a, Phys. Rev. Lett. 86, 2677.

Salis, G., Y. Kato, K. Ensslin, D. Driscol, A. C. Gossard and D. D. Awschalom, 2001b, Nature 414, 619.

Sanvito, S., P. Ordejón and N. A. Hill, 2001, Phys. Rev. B 63, 165206.

Sanvito, S and N. A. Hill, 2001, Appl. Phys. Lett. 78, 3493.

Sapega, V. F., T. Ruf and M. Cardona, 2000, Solid State Commun. 114, 573.

Sapega, V. F., T. Ruf and M. Cardona, 2001, Phys. Stat. Solidi b 226, 339.

Sato, K. and H. Katayama-Yoshida, 2000, Jpn. J. Appl. Phys. 39, L555.

Sato, K. and H. Katayama-Yoshida, 2001a, Jpn. J. Appl. Phys. 40, L651.

Sato, K. and H. Katayama-Yoshida, 2001b, Jpn. J. Appl. Phys. 40,
L334.

Sato, K. and H. Katayama-Yoshida, 2001c, Jpn. J. Appl. Phys. 40, L485.

Satoh, Y., N. Inoue, N. Nishikawa, J. Yoshino, Third Symposium on Physics and Application of Spin-Related Phenomena in Semiconductors, 17-18 Nov. 1997, Sendai, Japan, p.23.

Satoh, Y., D. Okazawa, A. Nagashima and J. Yoshino, 2001, Physics E 10, 196.

Schairer, W. and M. Schmidt, 1974, Phys. Rev. B 10, 2501.

Schliemann, J., J. König, H. -H. Lin and A. H. MacDonald, 2001a, Appl. Phys. Lett. 78, 1550

Schliemann, J., J. König and A. H. MacDonald, 2001b, Phys. Rev. B 64, 165201.

Schott, G. M., W. Faschinger and L. W. Molenkamp, 2001, Appl. Phys. Lett. 79, 1807

Schneider, J., U. Kaufmann, W. Wilkening, M. Baeum;er and F. Kohl, 1987, Phys. Rev. Lett. 59, 240.

Schulthess, T. C. and W. H. Butler, 2001, J. Appl. Phys. 89, 7021.

Semenov, Y. G. and V. A. Stephanovich, 2001, cond-mat/0106406.

Shen, A., H. Ohno, F. Matsukura, Y. Sugawara, N. Akiba, T. Kuroiwa, A. Oiwa, A. Endo, S. Katsumoto and Y. Iye, 1997a, J. Cryst. Growth 175/176, 1069 .

Shen, A., Y. Horikoshi, H. Ohno and S. P. Guo, 1997b, Appl. Phys. Lett. 71, 1540 .

Shen, A., F. Matsukura, Y. Sugawara, T. Kuroiwa, H. Ohno, A. Oiwa, A. Endo, S. Katsumoto and Y. Iye, 1997c, Appl. Surf. Sci. 113/114, 183.

Shen, A., H. Ohno, F. Matsukura, Y. Sugawara, Y. Ohno, N. Akiba and T. Kuroiwa, 1997d, Jpn. J. Appl. Phys. 36, L73.

Shen, A., F. Matsukura, S. P. Guo, Y. Sugawara, H. Ohno, M. Tani, H. Abe and H. C. Liu, J. Cryst. 1999, Growth 201/202, 673.

Shibata, N., A. Ohki and A. Katsui, 1988, J. Cryst. Growth 93. 703.

Shioda, R, K. Ando, T. Hayashi and M. Tanaka, 1998, Phys. Rev. B 58 1100 .

Shirai, M., 2001, Physica 10, 143.

Shirai, M, T. Ogawa, I. Kitagawa and N. Suzuki, 1998, J. Magn. Magn. Mater. 177-181, 1383.

Shimizu, H., T. Hayashi, M. Takenaka, Y. Nakano, M. Tanaka, K. Ando, 1998, Fourth Symposium on Physics and Application of Spin Related Phenomena in Semiconductors, 3-4 Dec 1998, Sendai, Japan, p. 53

Shimizu, H, T. Hayashi, T. Nishinaga and M. Tanaka, 1999, Appl. Phys. Lett. 74, 398.

Shono, T., T. Hasegawa, T. Fukumura, F. Matsukura and H. Ohno, 2000, Appl. Phys. Lett. 77, 1363.

Slupinski, T., H. Munekata and A. Oiwa, 2002a, Appl. Phys. Lett. 80, 1592.

Slupinski, T, A. Oiwa, S. Yanagi and H. Munekata, 2002b, J. Cryst. Growth, to be published.

Sonoda, S., S. Shimizu, T. Sasaki, Y. Yamamoto and H. Hori, 2001, cond-mat/0108159.

Soo, Y. L., S. W. Huang, Z. H. Ming, Y. H. Kao, H. Munekata and L. L. Chung, 1996, Phys. Rev. B 58, 4905.

Soo, Y. L., G. Kioseoglou, S. Huang, S. Kim, Y. H. Kao, Y. Takatani, S. Haneda and H. Munekara, 2001, Phys. Rev. B 63, 195209.

Soo, Y. L., G. Kioseoglou, S. Kim, S. Huang, Y. H. Kao, S. Kuwabara, S Owa, T. Kondo and H. Munekara, 2001b, Appl. Phys. Lett. 79, 3926.

Story, T., R. R. Gałazka, R. B. Frankel and P. A. Wolf, 1986, Phys. Rev. Lett. 56. 777.

Sugawara, Y, N. Akiba, T. Kuroiwa, F. Matsukura, A. Shen and H. Ohno, 1997, presented at The 3rd Symposium on the Physics and Applocation of Spin-Related Phenomena in Semiconductors, (Sendai, Japan, Nov. 17-18, 1997) pp. 30-33.

Szczytko, J., W. Mac, A. Stachow, A. Twardowski, P. Becla and J. Tworzydło, 1996, Solid State Comunn. 99, 927.

Szczytko, J., A. Twardowski, K. Świątek, M. Palczewska, M. Tanaka, T. Hayashi and K. Ando, 1999a, Phys. Rev. B 60, 8304.

Szczytko, J., W. Mac, A. Twardowski, F. Matsukura and H. Ohno, 1999b, Phys. Rev. B 59, 12935.

Szczytko, J., A. Twardowski, M. Palczewska, R. Jabłoński, J. Furdyna and H. Munekata, 2001a, Phys. Rev. B 63, 085315.

Szczytko, J., W. Bardyszewski and A. Twardowski, 2001b, Phys. Rev. B 64, 075306.

Szuszkiewicz, W., E. Dynowska, B. Hennion, F. Ott, M. Jouanne, J.-F. Morhange, M. Karlsteen and J. Sadowski, 2001, Acta Phys. Polon A. 100, 335 .

Takamura, K. F. Matsukura, Y. Ohno and H. Ohno, 2001, J. Appl. Phys. 89, 7024 .

Tanaka, M., 1998, Physica E 2, 372.

Tanaka, M. and Y. Higo, 2001, Phys. Rev. Lett. 87, 026602. 
Tazima, M, K. Yamamoto, D. Okazawa, A. Nagashima and J. Yoshino, 2001a, Physica E 10, 186.

Tazima, M., A. Nagashima, K. Yamamoto, D. Okazawa and J. Yoshino, 2001b, Extend Abstracts (The 48th Spring Meeting, The Japan Society of Applied Physics), p.1322.

Theodoropolpu, N., A. F. Hebard, M. E. Overberg, C. R. Abernathy, S. J. Pearton, S. N. G. Chu and R. G. Wilson, 2001a, Appl. Phys. Lett. 78, 3475 .

Theodoropolpu, N., A. F. Hebard, S. N. G. Chu, M. E. Overberg, C. R. Abernathy, S. J. Pearton, R. G. Wilson and J. M. Zavada, 2001b, Appl. Phys. Lett. 79, 3452.

Theodoropolpu, N., A. F. Hebard, M. E. Overberg, C. R. Abernathy, S. J. Pearton, S. N. G. Chu and R. G. Wilson, 2002, cond-mat/0201492.

Tokura, Y., 2000, in: Clossal Magnetoresistive Oxides, ed. Y. Tokura (Gordon \& Breach Science Publishers, London).

Tokura, Y. and Y. Tomioka, 1999, J. Magn. Magn. Matter. 200, 1.

Tsuruoka, T., R. Tanimoto, N. Tachikawa, S. Ushioda, F. Matsukura and H. Ohno, 2000, in: Proceedings 25th International Conference on Physics of Semiconductors, Sep. 17-22, Osaka, Japan, N. Miura and T. Ando, eds. (Springer-Verlag Berlin Heidelberg 2001) p 245.

Tsuruoka, T., R. Tanimoto, N. Tachikawa, S. Ushioda, F. Matsukura and H. Ohno, 2002, Solid State Commun. 121, 79.

Ueda, K., H. Tabata and T. Kawai, 2001, Appl. Phys. Lett. 79, 988.

Ueda, S., S. Imada, T. Muto, Y. Saitoh, S. Suga, F. Matsukura and H. Ohno, 2001, Physica E 10, 210.

Van Esch, A., W. Van Roy, J. De Boeck, I. François, H. Bender, G. Borghs, L. Van Bockstal, R. Bogaerts and F. Herlach, 1995, Mater. Sci. Forum 182-184, 623.

Van Esch, A., L. Van Bockstal, J. De Boeck, G. Verbanck, A. S. van Sternbergen, P. J. Wellman, B. Grietens, R. Begaerts, F. Herlach and G. Borghs, 1997, Phys. Rev. B 56, 13103.

van Schlifgaarde, M. and O. N. Mryasov, 2001, Phys. Rev. B 63 233205.

Van Stapele, R. P., 1982, in: Handbook on Magnetic Materials, Vol. 3, ed. K. H. J. Bushow (North-Holland, Amsterdam), p. 603-745.

von Molnár, S., H. Munekata, H. Ohno and L. L. Chang, 1991, J. Magn. Magn. Mater. 93, 356.

Vrijen, R., E. Yablonovitch, K. Wang, H. W. Jiang, A. Balandin, V. Roychowdhury, T. Mor and D. DiVincenzo, 2000, Phys. Rev, A 62, 012306.

Vurgaftman, I. And J. R. Meyer, 2001, Phys. Rev. B 64, 245207.

Wahl, U., A. Vantomme, G. Langousche, J. G. Correia, L. Peralta and The ISOLDE Collaboration, 2001, Appl. Phys. Lett. 78, 3217.

Walukiewicz, W, 1988, Phys. Rev. B 37, 4760.

Wei, S. -H.and A. Zunger, 1993, Phys. Rev. B 48, 6111.

Wiley, J. D., 1975, in: Semiconductors and Semimetals, Vol. 10, eds R.K. Willardson and A.C. Beer (Academic Press, New York), p. 173.

Wojtowicz, T., T. Dietl, M. Sawicki, W. Plesiewicz and J. Jaroszyński, 1986, Phys. Rev. Lett. 56, 2419.

Wolf, S. A., 2000, J. Supeconductivity 13, 195, and other papers in the same issue.

Wolf, S., D. D. Awshalom, R. A. Buhrman, J. M. Daughton, S. von Molnár, M. L. Roukes, A. Y. Chtchelkanova and D. M. Treger, 2001, Science 294, 1488.

Wolff, P. A., R. N. Bhatt and A. C. Durst, 1996, J. Appl. Phys. 79, 5196.

Yamada, M., K. Ono, M. Mizuguchi, J. Okabayashi, T. Mano, M. Oshima and H. Akinaga, 2001, Extend Abstracts (Fall Meeting, The Japan Society of Applied Physics), p. 1011; Seventh Symposium on Physics and Application of Spin Related Phenomena in Semiconductors, 17-18 Dec 2001, Yokohama, Japan, p. 60.

Yamamoto, T. and H. Katayama-Yoshida, 1999, Fifth Symposium on Physics and Application of Spin Related Phenomena in Semiconductors, 16-17 Dec 1999, Sendai, Japan, p. 170.

Yanagi, S., A. Oiwa, Y. Kitamoto, T. Slupinski and H. Munekata, 2001, Seventh Symposium on Physics and Application of Spin Related Phenomena in Semiconductors, 17-18 Dec 2001, Yokohama, Japan, p. 37.

Yang, J., H. Yasuda, S. Wang, F. Matsukura, Y. Ohno and H. Ohno, 2000, Appl. Surf. Sci. 166, 242.

Yasuda, H. and H. Ohno, 1999, Appl. Phys. Lett. 74, 3275.

Ye, J., Y. B. Kim, A. J. Millis, B. I. Shraiman, P. Majumdar and Z. Tešanović, 1999, Phys. Rev. Lett. 83, 3737.

Zając, M., R. Dorandziński, J. Gosk, J. Szczytko, M. Lefeld-Sosnowska, M. Kamińska, A. Twardowski, M. Palczewska, E. Grzanka and W. Gębicki, 2001a, Appl. Phys. Lett. 78, 1276.

Zając, M., J. Gosk, M. Kamińska, A. Twardowski, T. Szyszko and S. Podsiadło, 2001b, Appl. Phys. Lett. 79, 2432.

Zener, C., 1951a, Phys. Rev. 81, 440.
Zener, C., 1951b, Phys. Rev. 82, 403.

Zener, C., 1951c, Phys. Rev. 83, 299.

Zhang, F. C. and T. M. Rice, 1988, Phys. Rev. B 37, 3759

Zhang, S., 2000, Phys. Rev. Lett. 85, 393.

Zhao, J. H., F. Matsukura, K. Takamura, E. Abe, D. Chiba and H. Ohno, 2001, Appl. Phys. Lett. 79, 2276.

Zhao, J. H., F. Matsukura, E. Abe, Y. Ohno, K. Takamura and H. Ohno, 2002, J. Cryst. Growth, to be published.

Zhao, Y. -J., W. T. Geng, K. T. Park and A. J. Freeman, 2001a, Phys. Rev. B 64, 035207.

Zhao, Y. -J., W. T. Geng, A. J. Freeman and T. Oguchi, 2001b, Phys. Rev. B 63, 201202.

Ziese, M. and M. J. Thornton (eds.), 2001, Spin Electronics (Springer, 2001).

Zunger, A., 1986, in: Solid State Physics, Vol. 39, eds H. Ehrenreich and D. Turnbull (Academic Press, New York), 275.

Žutić, I., J. Fabian and S. Das Sarma, 2001, Phys. Rev. B 64, 121201. 